UTTG-94-1

LMU-TPW-94-1

January 1994

\title{
Field Dependent Gauge Couplings in Locally Supersymmetric Effective Quantum Field Theories ${ }^{\star}$
}

\author{
VADIM KAPLUNOVSKY $^{\dagger}$ \\ Theory Group, Dept. of Physics, University of Texas \\ Austin, TX 78712, USA \\ and \\ JAN LOUIS \\ Sektion Physik, Universität München \\ Theresienstrasse 37, D-80333 München, Germany \\ Dedicated to the Memory of Brian Warr
}

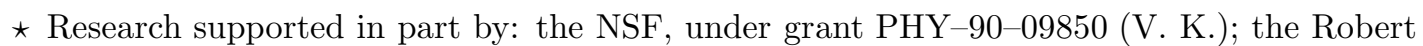
A. Welch Foundation (V. K.); the Heisenberg Fellowship of the DFG (J. L.); the NATO, under grant CRG 931380 (both authors).

$\dagger$ Email: vadim@bolvan.ph.utexas.edu

$\ddagger$ Email: jlouis@lswes8.1s-wess.physik.uni-muenchen.de
} 


\begin{abstract}
We investigate the field dependence of the gauge couplings of locally supersymmetric effective quantum field theories. We find that the Weyl rescaling of supergravity gives rise to Wess-Zumino terms that affect the gauge couplings at the one-loop level. These Wess-Zumino terms are crucial in assuring supersymmetric consistency of both perturbative and non-perturbative gauge interactions. At the perturbative level, we distinguish between the holomorphic Wilsonian gauge couplings and the physically-measurable momentum-dependent effective gauge couplings; the latter are affected by the Konishi and the super-Weyl anomalies and their field-dependence is non-holomorphic. At the non-perturbative level, we show how consistency of the scalar potential generated by infrared-strong gauge interactions with the local supersymmetry requires a very specific form of the effective superpotential. We use this superpotential to determine the dependence of the supersymmetric condensates of a strongly interacting gauge theory on its (field-dependent) Wilsonian gauge coupling and the Yukawa couplings of the matter fields. The article concludes with the discussion of the field-dependent non-perturbative phenomena in the context of string unification.
\end{abstract}




\section{Introduction and Summary}

A unified theory of all particle interactions must ultimately account for the gravitational force, and within the context of General Relativity a supersymmetric theory must necessarily be locally supersymmetric. Locally supersymmetric quantum field theories are non-renormalizable but nevertheless serve as effective quantum field theories (EQFTs) for energies well below the Planck scale. Physics of ultra-high energies of the order $O\left(M_{\mathrm{Pl}}\right)$ and beyond has to be governed by some theory which is outside the conventional framework of local quantum fields in four-dimensional spacetime, perhaps by a string theory. The locally supersymmetric EQFT acts as a bridge over the energy chasm between the string theory at the Planck scale and the non-supersymmetric Standard Model at the weak scale. At the high-energy end of this bridge, the low-energy limit of the string theory determines the high-energy couplings of the EQFT; couplings at lower energies are then controlled by the field-theoretical renormalization group equations. The intermediate-energy phenomena — which presumably trigger spontaneous breakdown of the local supersymmetry - are also governed by the EQFT.

A distinct feature of the string unification is that all couplings of the EQFT depend on massless scalar fields $\Phi^{i}$ called 'moduli'. From the string point of view, vacuum expectation values $(\mathrm{VEVs})$ of the moduli parametrize continuously degenerate families of string vacua. From the EQFT point of view, the moduli are massless neutral scalars that have exactly flat potentials to all orders of the perturbation theory; consequently, conventionally normalized moduli VEVs can be as big as $M_{\mathrm{Pl}}$. This article is about the moduli dependence of the gauge couplings of the EQFT and its consequences for the nonperturbative phenomena such as gaugino condensation. Our analysis is entirely field-theoretical in that we make no assumptions about the nature of the unified theory at ultra-high energies and only insist that its low-energy limit is a four-dimensional locally supersymmetric EQFT with moduli. In particular, we do not assume any properties of the moduli that do 
not follow from their origin in the Planck-scale vacuum degeneracy and from the local supersymmetry of the EQFT. Thus, although our assumptions are inspired by the superstring, our results apply equally to other unified theories with similar low-energy features.

Any discussion of the couplings of an effective quantum field theory must distinguish between two very different kinds of renormalized couplings ${ }^{[1]}$ : First, there are coefficients of the quantum operators in the Lagrangian of the effective theory from which the ultra-high momentum modes of the quantum fields are integrated out while the remaining modes are fully quantized. These coefficients depend on the scale $\Lambda$ of the ultraviolet cutoff and we call them the Wilsonian couplings since they renormalize according to the Wilsonian renormalization group. ${ }^{[2]}$ Second, there are effective couplings associated with physical processes; for example, Coulomblike scattering of charged particles defines a momentum-dependent gauge coupling $\{g(p)\}$. We refer to these couplings as the effective couplings and denote them with curly braces ' \{\} '. Unlike the Wilsonian couplings, the effective couplings depend not on the ultraviolet cutoff scale but on the momenta of the particles involved; thus, they do not correspond to any local effective Lagrangian. The dependence of the effective couplings on the overall momentum scale is governed by the CallanSymanzik equations, which resemble the Wilsonian renormalization group equations but have different physical meaning and different $\beta$-functions beyond one loop.

The analytic properties of the moduli dependence of the Wilsonian and the effective gauge couplings are very different from each other. ${ }^{[3]}$ We find (in section 2) that a properly defined Wilsonian gauge coupling $1 / g_{W}^{2}$ of a locally supersymmetric EQFT must be given by the real part of a holomorphic function $f^{W}(\Phi)$, plus a Wess-Zumino term originating in the Weyl rescaling ${ }^{[4-6]}$ (which is needed to make the $N=1$ supergravity consistent with the ordinary Einsteinian gravity). The Wilsonian gauge couplings renormalize only at one-loop ${ }^{[1,7]}$ and thus both the holomorphic functions $f^{W}(\Phi)$ and the Wess-Zumino terms are completely deter- 
mined at the one-loop level of the EQFT. This fact leads to exact (i.e., correct to all orders of the perturbation theory) transformation rules for the $f^{W}$ under global symmetries of the EQFT, including anomalous and Kähler symmetries (section 3.2). Similarly, for EQFTs with thresholds at energies smaller than $M_{\mathrm{Pl}}$ but bigger than the supersymmetry breaking scale, we derive exact threshold corrections to the Wilsonian gauge couplings (section 3.3).

The analytic properties of the effective gauge couplings are more complicated. While the Wilsonian gauge couplings are related by supersymmetry (SUSY) to the $\theta$-angles of the EQFT, the effective gauge couplings are related to the effective axionic couplings, which (in a theory with massless charged fermions) cannot be summarized in any kind of a local effective Lagrangian; consequently, the moduli dependence of the effective gauge couplings is non-holomorphic. ${ }^{[8]}$ From a different point of view, the non-holomorphicity follows from the two anomalies of locally supersymmetric EQFTs ${ }^{[9-11]}$ : The Konishi anomaly ${ }^{[12]}$ associated with noncanonically normalized charged fields and the super-Weyl anomaly peculiar to local SUSY. Shifman and Vainshtein ${ }^{[1]}$ used the Konishi anomaly to integrate the CallanSymanzik equations for the effective gauge couplings of a rigidly supersymmetric gauge theory. In this article, we extend their results to locally supersymmetric EQFTs (section 3.1) and use them to determine the moduli dependence of the confinement scales of the asymptotically-free gauge interactions (section 4).

String unification often gives rise to 'hidden' asymptotically-free and thus infrared-strong gauge interactions that do not affect the known particles, and the moduli dependence of their confinement scales leads to a non-perturbative effective potential for the moduli fields. ${ }^{[13,14]}$ In section 4 we calculate this effective potential by integrating out all the strongly interacting degrees of freedom of a locally supersymmetric gauge theory, ${ }^{[15]}$ including the charged (non-singlet) matter multiplets, if present. The Wess-Zumino term in the Wilsonian gauge coupling plays a crucial role in the consistency of the resulting potential with the local supersymmetry of the EQFT. This consistency also imposes severe constraints on the moduli depen- 
dence of the non-perturbative condensates of the theory: All the supersymmetric VEVs of the theory (including the gaugino condensate $\langle\lambda \lambda\rangle$ ) are controlled by a holomorphic effective superpotential.

The effective superpotential is comprised of the tree-level superpotential of the EQFT, of its Wilsonian gauge couplings and of the non-perturbative terms, which depend only on the gauge quantum numbers of the matter fields but are totally blind to all coupling parameters of the EQFT. For many gauge theories, the exact form of the non-perturbative terms is completely determined by the supersymmetric consistency conditions of the moduli's potential. ${ }^{[16-24,9]}$ For example, SQCD-like theories with more colors than flavors give rise to the TaylorVeneziano-Yankielowicz superpotential ${ }^{[16]}$ without any further perturbative or nonperturbative corrections (that are not supressed by $M_{\mathrm{Pl}}$ ), while for $N_{\text {flavor }}=N_{\text {color }}$ the effective superpotential is exactly as conjectured in ref. [25]. These, and a few more examples are discussed in section 4.3 .

In the absence of non-gauge couplings, supersymmetric gauge theories with massless matter multiplets often suffer from non-perturbative 'run-away' vacuum instabilities. $^{[17]}$ In the context of string unification, the charged (non-singlet) matter fields are massless, but the EQFT may have Yukawa couplings that prevent the run-away and keep all the non-perturbative VEVs near the confinement scale of the strong gauge interactions. Alternatively, the run-away may be stopped by the non-renormalizable (quartic or higher-order) couplings in the superpotential of the EQFT. In this case, some scalar VEVs dynamically produce an intermediate scale between the confinement scale and the Planck scale. In section 4.4 we give examples of both behaviors.

Situations where the run-away is not stopped by the tree-level superpotential of the EQFT are discussed in section 4.5. There we also discuss the stability or instability of the moduli VEVs once a non-perturbative effective potential is produced by strong gauge interactions. Our discussion of these issues is general rather than 
specific; our goal is to survey various scenarios that may happen in the context of the string unification (or of any other kind of unification with similar sub-Planck energy properties). Very briefly, we discuss how the spontaneous breakdown of supersymmetry fits into those scenarios; the phenomenology of the supersymmetry breaking among the observable particles is presented in a separate article. ${ }^{[26]}$

\section{Locally Supersymmetric Quantum Effective Field Theories}

\subsection{The Classical Action.}

As a prelude to locally supersymmetric EQFTs, consider the classical action of a generic locally supersymmetric effective field theory. In the standard superfield formalism, ${ }^{[4-6]}$ one has

$$
\mathcal{S}=\frac{-3}{\kappa^{2}} \int d^{8} z \mathbf{E} \exp \left(-\frac{1}{3} \kappa^{2} K\right)+\int d^{8} z \mathcal{E}\left[W+\frac{1}{4} f_{(a)(b)} \mathcal{W}^{(a) \alpha} \mathcal{W}_{\alpha}^{(b)}\right]+\text { h. c. }
$$

plus higher-derivative terms. Superfields of the effective field theory correspond to light particles of the string theory (or some other unified theory). We distinguish between two kinds of chiral superfields, namely the moduli $\Phi^{i}$ and the 'matter' superfields $Q^{I}$. The VEVs of the scalar components of the moduli parametrize the degeneracy of the classical vacua of the unified theory and can be as big as $M_{\mathrm{Pl}}\left(\kappa^{2}=\frac{8 \pi}{M_{\mathrm{Pl}}^{2}}\right)$; consequently, all moduli are neutral with respect to the entire four-dimensional gauge symmetry. Note that although the moduli originate at the unification scale $O\left(M_{\mathrm{Pl}}\right)$, they are massless and thus should be retained in the low-energy EFT, unlike the genuinely superheavy fields that simply decouple from the low-energy physics. The 'matter' superfields contain the ordinary scalars (e.g., Higgses) and fermions (e.g., quarks and leptons) of the theory and their superpartners. Scalar superfields that are charged with respect to a 'hidden' gauge symmetry are also treated as 'matter'. The kinetic-energy terms for the scalar fields and their superpartners are encoded in the Kähler function $K$ - a gauge-invariant 
real analytic function of all the chiral superfields of the theory. Expanding $K$ in powers of the matter superfields, we have

$$
K(\Phi, \bar{\Phi}, Q, \bar{Q})=K_{\bmod }(\Phi, \bar{\Phi})+Z_{\bar{I} J}(\Phi, \bar{\Phi}) \bar{Q}^{\bar{I}} e^{2 V} Q^{J}+\cdots
$$

where the $\cdots$ stand for higher-order terms, which are irrelevant for the present investigation. $Z_{\bar{I} J}$ is the moduli-dependent normalization matrix of the kineticenergy terms for the matter fields; the normalization matrix for the moduli fields themselves is given by

$$
G_{\bar{\imath} j}=\frac{\partial^{2} K_{\bmod }}{\partial \bar{\Phi}^{\bar{\imath}} \partial \Phi^{j}}
$$

$W$ is the superpotential - a gauge-invariant holomorphic function of the chiral superfields $\Phi^{i}$ and $Q^{I}$ controlling their masses and Yukawa interactions. Similar to the Kähler function, it expands into

$$
W(\Phi, Q)=\frac{1}{2} M_{I J}(\Phi) Q^{I} Q^{J}+\frac{1}{3} Y_{I J K}(\Phi) Q^{I} Q^{J} Q^{K}+\cdots
$$

where $M_{I J}$ is the un-normalized mass matrix for the matter fields while $Y_{I J K}$ are their un-normalized Yukawa couplings. A non-vanishing $M_{I J}$ matrix only arises in EFTs with an explicit mass scale well below $M_{\mathrm{Pl}}$ while fields with $O\left(M_{\mathrm{Pl}}\right)$ masses are not part of a low-energy EFT. Perturbatively, there is no such scale in the string theory and hence generically no $M_{I J}$ arise in EFTs based upon the superstring. However, since we expect some kind of a non-perturbative mechanism to generate the weak scale, we also allow for the possibility that the same mechanism generates an intermediate scale. (Section 3.3 is devoted to the effects of those mass terms on the low-energy gauge couplings.)

\footnotetext{
$\star$ Certain string states do have $\Phi$-dependent masses that can vary continuously between zero and $M_{\mathrm{Pl}}$. In section 4.5 we discuss their role in the low energy EFT.
} 
The superfield $V \equiv V^{(a)} T_{(a)}$ in eq. (2.2) contains the gauge fields $A_{m}^{(a)}$ and their superpartners, ${ }^{\dagger}$ while $\mathcal{W}_{\alpha}^{(a)}$ in eq. (2.1) are the superfield analogues of the field strengths $F_{m n}^{(a)}$; specifically $\mathcal{W}_{\alpha} \equiv \mathcal{W}_{\alpha}^{(a)} T_{(a)}=\left(\frac{1}{8} \overline{\mathcal{D}}^{2}-\mathcal{R}\right) e^{-2 V} \mathcal{D}_{\alpha} e^{2 V}$. The coefficients $f_{(a)(b)}$ are the supersymmetric gauge couplings of the theory; in the component-field expansion of (2.1), the kinetic-energy terms for the gauge fields are

$$
-\frac{1}{4} \int d^{4} x \sqrt{-g}\left(\operatorname{Re} f_{(a)(b)} F_{m n}^{(a)} F^{(b) m n}-\frac{1}{2} \operatorname{Im} f_{(a)(b)} \epsilon^{m n p q} F_{m n}^{(a)} F_{p q}^{(b)}\right) .
$$

Gauge invariance and local supersymmetry require the functions $f_{(a)(b)}(\Phi, Q)$ to be both gauge-covariant and holomorphic. Furthermore, in the low-energy limit,

$$
\begin{aligned}
f_{(a)(b)}(\Phi, Q) & =f_{(a)(b)}(\Phi)+\cdots \\
& =\delta_{(a)(b)} \cdot f_{a}(\Phi)+\cdots
\end{aligned}
$$

where the $\cdots$ stand for the irrelevant terms and the index $a$ (without parentheses) refers to a particular simple factor of the gauge group $G=\prod_{a} G_{a}$ that contains the generator $T^{(a)}$; were the entire gauge group simple, we would have only one $f(\Phi)$. The second equality here follows from the gauge-covariance of $f_{(a)(b)}$ and the neutrality of the moduli with respect to all gauge symmetries that remain unbroken below the unification scale. The relation (2.6) makes for a particularly

$\dagger$ For notational simplicity, we are suppressing the gauge indices of the matter superfields, so $Q^{I}$ and $\bar{Q}^{\bar{I}}$ actually stand for the entire irreducible multiplets of the gauge group. In more explicit notations, the second term on the right hand side of eq. (2.2) becomes

$$
Z_{\bar{I} J}(\Phi, \bar{\Phi}) \bar{Q}_{s}^{\bar{I}}\left[\exp \left(2 V^{(a)} T_{(a)}\right)\right]_{s^{\prime}}^{s} Q^{J s^{\prime}}
$$

where $s, s^{\prime}$ are the gauge indices and $\left[T_{(a)}\right]_{s^{\prime}}^{s}$ are the explicit hermitian matrices realizing the Lie-algebra generators $T_{(a)}$ in the appropriate representation of the gauge group. In either notation, the matrix $Z_{\bar{I} J}$ is block diagonal - the 'flavor' indices $\bar{I}$ and $J$ must agree with respect to the kind of a gauge-group multiplet formed by respective matter fields, but different 'generational' copies of the same kind of multiplet may freely mix with each other. 
simple interpretation of the gauge-field Lagrangian (2.5) in terms of the ordinary moduli-dependent couplings $g_{a}(\Phi, \bar{\Phi})$ and moduli-dependent vacuum angles $\theta_{a}(\Phi, \bar{\Phi})$, which are related to the supersymmetric couplings $f_{a}(\Phi)$ via

$$
g_{a}^{-2}(\Phi, \bar{\Phi})=\operatorname{Re} f_{a}(\Phi), \quad \theta_{a}(\Phi, \bar{\Phi})=-8 \pi^{2} \operatorname{Im} f_{a}(\Phi)
$$

Note that holomorphicity of the functions $f_{a}(\Phi)$ implies that both $1 / g_{a}^{2}$ and $\theta_{a}$ are real harmonic functions of the complex moduli fields and that each of the two functions determines the other up to an overall constant.

Finally, the gravitational fields and their superpartners comprise the vielbein $E_{M}^{A}$. These superfields appear in the Lagrangian (2.1) both explicitly, through the densities $\mathbf{E}=\operatorname{det}\left(E_{M}^{A}\right)$ and $\mathcal{E}=\mathbf{E} / 2 \mathcal{R}(\mathcal{R}$ is the scalar superspace-curvature superfield), and also implicitly, through the commutation relations of the supercovariant derivatives $\mathcal{D}_{A}=E_{A}^{M} \mathcal{D}_{\mathcal{M}}$. . The latter commutation relations are subject to the so-called torsion constraints ${ }^{[5,6]}$; as the result of these constraints, the first term in (2.1) gives not only the kinetic-energy terms for the scalar fields and their superpartners, but also for the gravitational fields themselves.

Unfortunately, extracting those kinetic-energy terms from eq. (2.1) is not so easy. A naive interpretation of the lowest components $\left.\hat{e}_{m}^{a} \equiv E_{m}^{a}\right|_{\Theta=\bar{\Theta}=0}$ of the vielbein as the ordinary vierbein and the corresponding metric tensor $\hat{g}_{m n} \equiv \hat{e}_{m}^{a} \eta_{a b} \hat{e}_{n}^{b}$ as the physical (Einsteinian) metric of spacetime results in a field-dependent gravitational coupling $\hat{G}_{N}=\left(\kappa^{2} / 8 \pi\right) \cdot \exp \left(-\frac{1}{3} \kappa^{2} K\right)$, as well as non-Kählerian kineticenergy terms for the scalar fields and a host of other complications. The usual procedure for disentangling supergravity from other fields is known as the Weyl

$\star M$ here is the curved superspace index: $z^{M}=\left(x^{m}, \Theta^{\mu}, \bar{\Theta}^{\dot{\mu}}\right) . \quad A$ is the flat local superLorentz index, running over both Lorentz vectors and Lorentz spinors. Elsewhere in this article, $\alpha, \beta, \ldots$ and $\dot{\alpha}, \dot{\beta}, \ldots$ are local Lorentz spinor indices while $a, b, \ldots$ are local Lorentz vector indices or indices labelling simple factors of the gauge group (as in eq. (2.7)); we apologize for the coincidence. $\overline{\mathcal{D}}_{\dot{\alpha}}$ is the same as $\mathcal{D}_{\dot{\alpha}}$ and $\overline{\mathcal{D}}^{2}$ is short-hand for $\overline{\mathcal{D}}^{\dot{\alpha}} \overline{\mathcal{D}}_{\dot{\alpha}}$ while $\mathcal{D}^{2}$ is short-hand for $\mathcal{D}^{\alpha} \mathcal{D}_{\alpha}$. 
rescaling because it involves a rescaled spacetime metric ${ }^{[4,5]}$

$$
g_{m n}=\hat{g}_{m n} \cdot e^{-\frac{1}{3} \kappa^{2} K}
$$

All the fermionic fields of the theory are also rescaled by appropriate exponentials of $\kappa^{2} K$; the purpose of this rescaling is to assure that the bosons and the fermions belonging to the same supermultiplet are normalized in the same way.

Let us close this section with a few technical remarks. The action (2.1) is not completely generic. In principle, the superpotential $W$ could contain an O'Raifeartaigh term in addition to the terms listed in eq. (2.4); similarly, the Kähler function $K$ could also contain a Fayet-Iliopoulos term for one of the abelian gauge superfields. In superstring-based unified theories, whenever such terms occur, they are of the order $O\left(M_{\mathrm{Pl}}^{2}\right)$ and thus cause major rearrangements of the vacuum family right at the unification scale. Consequently, once the theory is expanded around the right vacuum family and the particles with $O\left(M_{\mathrm{Pl}}\right)$ masses are excluded from the low-energy EQFT, the O'Raifeartaigh and the Fayet-Iliopoulos terms disappear. For other unified theories, such terms might conceivably be present and have magnitudes much less than $M_{\mathrm{Pl}}^{2}$; however, they would not have any effect on the subject matter of this article. Thus, to avoid unnecessary complication of our notations, we chose to omit the O'Raifeartaigh and the Fayet-Iliopoulos terms from eqs. (2.2) and (2.4) and from all subsequent discussion. For the same reason, we have assumed that in theories with several abelian gauge symmetries $G_{a}$, the abelian gauge superfields $V^{(a)}$ do not mix with each other. If they do, the supersymmetric gauge coupling matrix $f_{(a)(b)}$ has non-diagonal terms in addition to $f_{a}$; generalizing our arguments to such non-diagonal couplings is completely straightforward.

On the other hand, we lose no generality by assuming that all scalars belong to chiral rather than linear superfields. In four spacetime dimensions, $N=1$ linear multiplets are always dual to chiral multiplets (with very specific couplings). For 
example, the dilaton of string theory can be treated as just another chiral modulus superfield $S$. (The special properties of the dilaton are discussed elsewhere. ${ }^{[10,11,27,28]}$ )

\subsection{The Weyl Compensator Formalism}

In the usual formalism of minimal supergravity, the Weyl rescaling is done in terms of component fields. However, in order to understand the anomalous quantum corrections to the classical action (2.1), we need a manifestly supersymmetric formalism, in which the Weyl rescaling is also supersymmetric. ${ }^{[2]}$

The simplest formalism of this kind makes use of the invariance of the torsion constraints of minimal supergravity and of the chirality constraints $\mathcal{D}_{\dot{\alpha}} \Phi^{i}=$ $\mathcal{D}_{\dot{\alpha}} Q^{I}=0$ under a local super-Weyl symmetry ${ }^{[30,31,5]}$

$$
\begin{aligned}
& E_{M}^{\alpha} \mapsto e^{(2 \bar{\tau}-\tau)} \cdot\left(E_{M}^{\alpha}-\frac{i}{2} \bar{\sigma}_{a}^{\dot{\beta} \alpha}\left(\mathcal{D}_{\dot{\beta}} \bar{\tau}\right) E_{M}^{a}\right), \quad E_{M}^{\dot{\alpha}} \mapsto e^{(2 \tau-\bar{\tau})} \cdot\left(E_{M}^{\dot{\alpha}}-\frac{i}{2} \bar{\sigma}_{a}^{\dot{\alpha} \beta}\left(\mathcal{D}_{\beta} \tau\right) E_{M}^{a}\right), \\
& E_{M}^{a} \mapsto e^{(\tau+\bar{\tau})} \cdot E_{M}^{a}, \quad \mathbf{E} \mapsto e^{2(\tau+\bar{\tau})} \cdot \mathbf{E}, \quad \mathcal{E} \mapsto e^{6 \tau} \cdot \mathcal{E}+\cdots, \quad \overline{\mathcal{E}} \mapsto e^{6 \bar{\tau}} \cdot \overline{\mathcal{E}}+\cdots, \\
& \mathcal{D}_{\alpha} \mapsto e^{(\tau-2 \bar{\tau})}\left(\mathcal{D}_{\alpha}-2\left(\mathcal{D}^{\beta} \tau\right) L_{\alpha \beta}\right), \quad \mathcal{D}_{\dot{\alpha}} \mapsto e^{(\bar{\tau}-2 \tau)}\left(\mathcal{D}_{\dot{\alpha}}-2\left(\mathcal{D}^{\dot{\beta}} \bar{\tau}\right) L_{\dot{\alpha} \dot{\beta}}\right), \\
& \Phi^{i} \mapsto \Phi^{i}, \quad Q^{I} \mapsto Q^{I}, \quad \bar{\Phi}^{\imath} \mapsto \bar{\Phi}^{\imath}, \quad \bar{Q}^{\bar{I}} \mapsto \bar{Q}^{\bar{I}}, \\
& V^{(a)} \mapsto V^{(a)}, \quad \mathcal{W}_{\alpha}^{(a)} \mapsto e^{-3 \tau} \cdot \mathcal{W}_{\alpha}^{(a)}, \quad \overline{\mathcal{W}}_{\dot{\alpha}}^{(a)} \mapsto e^{-3 \bar{\tau}} \cdot \overline{\mathcal{W}}_{\dot{\alpha}}^{(a)},
\end{aligned}
$$

parametrized by an arbitrary chiral superfield $\tau(z)$ and its anti-chiral conjugate $\bar{\tau}(z)$. Here $L_{\alpha \beta}$ and $L_{\dot{\alpha} \dot{\beta}}$ are the generators of the local Lorentz symmetry, the transformation rule for the $\mathcal{D}_{a}$ derivatives is not displayed as too complicated and not germane to the present discussion and the '...' stand for inhomogeneous terms in the chiral measures $\mathcal{E}$ and $\overline{\mathcal{E}}$ that do not contribute to integrals such as in (2.1). Although the scalar and the vector superfields remain invariant under (2.9), the definitions of their fermionic components change due to changing covariant super- 
derivatives $\mathcal{D}_{\alpha}$ and $\mathcal{D}_{\dot{\alpha}}$. In particular, the gauginos $\lambda_{\alpha}^{(a)}$ transform like

$$
\left.\lambda_{\alpha}^{(a)} \equiv \mathcal{W}_{\alpha}^{(a)}\right|_{\Theta=\bar{\Theta}=0} \mapsto \lambda_{\alpha}^{(a)} \cdot e^{-3 \tau}
$$

while the matter fermions $\Psi_{\alpha}^{I}$ transform like

$$
\left.\Psi_{\alpha}^{I} \equiv \mathcal{D}_{\alpha} Q^{I}\right|_{\Theta=\bar{\Theta}=0} \mapsto \Psi_{\alpha}^{I} \cdot e^{\tau-2 \bar{\tau}}
$$

As written, the action functional (2.1) is not super-Weyl invariant. However, this lack of symmetry can be easily remedied with the help of a chiral superfield $\varphi$ $\left(\mathcal{D}_{\dot{\alpha}} \varphi=0\right)$ that transforms under $(2.9)$ like ${ }^{[29]}$

$$
\varphi \mapsto e^{-2 \tau} \cdot \varphi
$$

This field is known as the Weyl compensator because formula (2.12) allows us to compensate for 'wrong' super-Weyl scaling properties of any term in the action functional by multiplying it by an appropriate power of $\varphi$ and/or $\bar{\varphi}$. Specifically, for the classical action (2.1), the Kähler function $K$, the superpotential $W$ and the supersymmetric gauge couplings $f_{a}$ are modified according to the following rules:

$$
\begin{aligned}
K(\Phi, \bar{\Phi}, Q, \bar{Q}) & \mapsto \widetilde{K}(\varphi, \bar{\varphi}, \Phi, \bar{\Phi}, Q, \bar{Q})=K-6 \kappa^{-2} \operatorname{Re} \log \varphi \\
W(\Phi, Q) & \mapsto \widetilde{W}(\varphi, \Phi, Q)=\varphi^{3} \cdot W(\Phi, Q), \\
f_{a}(\Phi) & \mapsto \tilde{f}_{a}(\varphi, \Phi)=\varphi^{0} \cdot f_{a}(\Phi) .
\end{aligned}
$$

Note that the compensating superfields $\varphi$ and $\bar{\varphi}$ are non-dynamical - according to eq. (2.13), their components appear in the extended action without derivatives and play purely auxiliary roles, which are two: To make the super-Weyl symmetry manifest, and also to break it spontaneously down to nothing. The breakdown is provided by $\langle\varphi\rangle \neq 0$; the actual value of this $\mathrm{VEV}$ is irrelevant since any $\varphi \neq 0$ can 
be super-Weyl-transformed into any desired function of $\left(x^{m}, \Theta^{\alpha}\right)$, as long as it is chiral and does not vanish. In particular, it is possible to set $\varphi \equiv 1$ and completely trivialize the extension (2.13-15); for other values of the $\varphi$ superfield, the action of the theory has a somewhat different form, but the physical content remains exactly the same - identical to the unextended theory defined by eq. (2.1).

The super-Weyl symmetry may be combined with the general supercoordinate invariance into the superconformal symmetry; accordingly, the $\varphi$ and the $\bar{\varphi}$ superfields are often called the superconformal compensators. However, viewed by itself, the transformation $(2.9) \&(2.12)$ is an internal spontaneously-broken supersymmetric abelian gauge symmetry with an unusual feature that the vielbein components are charged under (2.9). Another unusual feature is that the gauge superfield associated with this symmetry is not an independent vector superfield but a composite $\widetilde{K}(\varphi, \bar{\varphi}, \Phi, \bar{\Phi}, Q, \bar{Q})$ of the scalar superfields of the theory. Indeed, according to eqs. (2.9), (2.12) and (2.13), $\widetilde{K}$ transforms under super-Weyl transformations as

$$
\widetilde{K} \mapsto \widetilde{K}+\frac{6}{\kappa^{2}}(\tau+\bar{\tau})
$$

i.e., exactly like an abelian vector superfield $V$.

The particular super-Weyl parameters $\tau$ and $\bar{\tau}$ that reproduce the Weyl rescaling (2.8) of the metric and appropriate rescalings of the fermionic fields are

$$
\tau+\bar{\tau} \equiv-\left.\frac{\kappa^{2}}{6} K(\Phi, \bar{\Phi}, Q, \bar{Q})\right|_{\text {harmonic }}
$$

where the subscript 'harmonic' indicates that we are extracting the harmonic part of the real superfield on the right hand side, i.e., the components that can be put together into a sum of a chiral and an anti-chiral superfields. From the gaugetransformation point of view (2.16), eq. (2.17) corresponds to starting from the 'unitary' gauge $\varphi \equiv 1$ and changing it into the Wess-Zumino gauge for the com- 
posite $\widetilde{K}$ superfield:

$$
\begin{gathered}
\left.\widetilde{K}\right|_{\text {harmonic }}=0, \\
\text { i.e., } \quad \log \varphi+\log \bar{\varphi}=\left.\frac{1}{3} \kappa^{2} K(\Phi, \bar{\Phi}, Q, \bar{Q})\right|_{\text {harmonic }} .
\end{gathered}
$$

Similarly to ordinary gauge symmetries, the super-Weyl gauge $\varphi \equiv 1$ is manifestly supersymmetric but not quite physical while the Wess-Zumino gauge (2.18) is more physical but not quite supersymmetric. From the EQFT point of view, this means that the Wilsonian couplings of the theory defined in the $\varphi \equiv 1$ gauge have clear analytic properties due to manifest SUSY - for example, the Yukawa couplings $Y_{I J K}(\Phi)$ must be holomorphic functions of the moduli $\Phi^{i}$, - but the physical meaning of those couplings is obscured by the non-physical normalization of the metric and of the fermionic fields. On the other hand, in the WZ gauge (2.18), the normalization of the matter fields is clear, but the analytic properties of the Yukawa couplings are obscured by the fact that the holomorphic $Y_{I J K}(\Phi)$ have to be multiplied by a non-holomorphic factor $\exp \left(\frac{1}{2} \kappa^{2} K\right)$. Similarly, the Wilsonian gauge couplings $g_{a}^{-2}$ are harmonic functions of the moduli in the $\varphi \equiv 1$ gauge, but have more complicated moduli dependence in the Wess-Zumino gauge. This effect is anomalous - classically, eq. (2.7) holds in any super-Weyl gauge, — and is best understood in terms of a manifestly supersymmetric EQFT. It is for the sake of such manifest SUSY that we are using the Weyl compensator formalism in this article instead of the component-field Weyl rescaling of the usual formalism.

Actually, there is an alternative manifestly supersymmetric formalism which eliminates the Weyl rescaling altogether. In the so-called Kähler supergravity formalism, the local Lorentz symmetry is supplemented by an additional local $U(1)$ symmetry. ${ }^{[31]}$ This enlargement of the superspace structure group relaxes the torsion constraints of the ordinary minimal supergravity, which in turn leads to an extension of the super-Weyl symmetry (2.9). Consequently, as far as the $e^{-\kappa^{2} \widetilde{K} / 3}$ factor in the supergravity action is concerned, it becomes possible to impose at 
the superfield-level a gauge condition $\widetilde{K}=0$ instead of the component-level WessZumino gauge (2.18). For the purpose of this article we found the compensator formalism makes for a more transparent correspondence between local and rigid supersymmetries, and so we use this formalism throughout this article. Obviously, any other correct formalism should yield exactly the same physical results. ${ }^{[1]}$

\subsection{Supersymmetric Wilsonian Couplings of a Quantum EFT AND the Super-Weyl Anomaly.}

The Wilsonian couplings of an EQFT are the moduli-dependent coefficients of quantum operator products appearing in the action functional of the quantum theory. Similar to its classical counterpart, the Wilsonian action is spacetime-local, i.e., it is a $\int d^{4} x$ integral of a convergent power series in quantum fields and their derivatives. Consequently, manifest symmetries of an EQFT restrict the Wilsonian couplings of the theory in exactly the same way as the classical couplings are restricted by the classical symmetries. In particular, for an EQFT that maintains manifest local SUSY, manifest super-Weyl invariance and manifest background gauge invariance with respect to the ordinary gauge symmetries, the Wilsonian Yukawa couplings $\tilde{Y}_{I J K}^{W}(\Phi, \varphi)$ and the Wilsonian gauge couplings $\tilde{f}_{a}^{W}(\Phi, \varphi)$ are required to be holomorphic functions of the chiral moduli $\Phi^{i}$ and of the chiral compensator $\varphi$. Moreover, the $\varphi$-dependence of $\tilde{Y}_{I J K}^{W}$ and of $\tilde{f}_{a}^{W}$ is completely determined by the super-Weyl scaling dimensions of the corresponding terms in

\footnotetext{
$\star$ At energies well below $M_{\mathrm{Pl}}$, neither moduli quanta nor gravitons nor their superpartners have any renormalizable interactions with each other or any other light particles. In the Wilsonian approach to EQFTs with a cutoff $\Lambda \lesssim M_{\mathrm{Pl}}$, this means that all quantum operators involving those quanta are irrelevant and may be simply discarded from the action functional. Therefore, we treat the moduli and the gravitational superfields in the quantized version of the classical action (2.1) as purely classical background fields; in the compensator formalism, the same applies to $\varphi$ and $\bar{\varphi}$. Functions of those superfields appear in the quantized actions as mere coefficients - moduli-dependent coupling parameters - of the quantum operator products comprised of the matter and gauge superfields $Q^{I}, \bar{Q}^{\bar{I}}$ and $V^{(a)}$ and their derivatives. Perturbatively, this means that $\Phi^{i}, \varphi$ and the gravitational superfields may correspond to the external legs of 1PI Feynman graphs of the EQFT but not to their internal propagators.
} 
the Wilsonian action functional. The canonical scaling dimensions of those terms give us the classical eqs. (2.14) and (2.15); the goal of this section is to see how these equations are affected by the anomalous scaling dimensions characteristic of the quantum theory.

When studying the moduli-dependence or the $\varphi$-dependence of the Wilsonian couplings of an EQFT, one has to keep in mind that a complete description of an EQFT includes not only its action functional but also the ultraviolet cutoff scale $\Lambda$ and the exact manner in which the UV cutoff is implemented. Actually, only the relevant terms in the action functional are important, and the specifics of the cutoff can be absorbed into a finite renormalization of those terms; nevertheless, the 'bare' couplings of the theory have no precise meaning unless the cutoff is fully specified. In particular, it makes no sense to discuss moduli dependence of the bare gauge coupling of the theory without also discussing moduli dependence of the ultraviolet cutoff. Therefore, to give precise meaning to $\tilde{f}_{a}^{W}(\Phi, \varphi)$ and other Wilsonian couplings of the theory, we define them as the bare couplings of an explicitly UV-regulated theory whose cutoff does not depend on either the moduli or on the Weyl compensators and can be completely specified in terms of a single overall scale $\Lambda$. The difference between these Wilsonian couplings and the bare couplings of the same theory regulated in some other way is nothing but a finite renormalization of the theory.

In a rigidly supersymmetric EQFT with a manifestly supersymmetric ultraviolet cutoff, the Wilsonian superpotential does not renormalize. Moreover, if the cutoff also preserves manifest four-dimensional background gauge invariance, then

$\dagger$ In EQFTs with massless matter superfields, some two-loop contributions to the three-point Green's functions appear to renormalize the effective (but not the Wilsonian!) Yukawa couplings. $^{[32]}$ The exact physical meaning of these two-loop effects is controversial since they have singular dependence on the off-shell momenta of the three particles involved.

$\ddagger$ Note that a dimensionally-reduced background gauge invariance is insufficient. In the DR regularization scheme, not only the gauge couplings renormalize to all orders of perturbation theory, but the loop corrections do not even respect the classical $(N=1, d=4)$ supersymmetric relation $g_{a}^{-2}(\Phi, \bar{\Phi})=\operatorname{Re} f_{a}(\Phi)$. The reason for this failure is that the 
the Wilsonian supersymmetric gauge couplings $f_{a}^{W}$ renormalize only at the oneloop level. ${ }^{[1,7]}$ These no-renormalization theorems apply equally well to the locally supersymmetric EQFTs (the proof of this assertion is presented in Appendix A), which suggests the following strategy for calculating the quantum corrections to eqs. (2.14) and (2.15):

1. Start with an explicitly UV-regulated EQFT whose bare action and the cutoff are both manifestly invariant under local SUSY, as well as under other local symmetries, namely Lorentz, background gauge and super-Weyl. An example of such a cutoff is described in Appendix B.

2. Now consider the same theory with a different, $\varphi$-independent cutoff. The new cutoff should remain locally supersymmetric, Lorentz invariant and background gauge invariant, but in order to allow for the $\varphi$-independence, we give up on the super-Weyl invariance.

3. Because of the manifest super-Weyl symmetry, the $\varphi$-dependence of the bare couplings corresponding to the first cutoff is given by the unmodified classical eqs. (2.13-15). However, the Wilsonian couplings of the theory are the bare couplings corresponding to the second cutoff, and they differ from the first set of bare couplings by a finite renormalization.

Now is the time to make use of the no-renormalization theorems. Both cutoffs 1) and 2) being supersymmetric, the Yukawa couplings $\widetilde{Y}_{I J K}(\varphi, \Phi)$ do not renormalize at all and thus take exactly the same values for both cutoffs. Hence, the Wilsonian Yukawa couplings obey unmodified eq. (2.14):

$$
\tilde{Y}_{I J K}^{W}(\varphi, \Phi)=\varphi^{3} \cdot Y_{I J K}^{W}(\Phi)
$$

dimensionally-reduced gauge invariance allows for counter-terms that contain the ChernSimons tensor $\omega_{\ell m n}=\operatorname{tr}\left(A_{[\ell} F_{m n]}-\frac{2 i}{3} A_{[\ell} A_{m} A_{n]}\right)$ in such a manner that they cannot be re-expressed in terms of $\operatorname{tr}(F \tilde{F})$. Manifest supersymmetry does not forbid such counterterms either; instead, it relates them to corrections to the bare gauge couplings $g_{a}^{-2}(\Phi, \bar{\Phi})$ whose dependence on the moduli fields $\Phi^{i}$ and $\bar{\Phi}^{i}$ is non-harmonic. ${ }^{[33]}$ 
On the other hand, the supersymmetric gauge couplings $\tilde{f}_{a}(\varphi, \Phi)$ do renormalize, but only at the one-loop level. Thus, it becomes a straightforward exercise in superfield Feynman rules to calculate the entire finite renormalization of those couplings due to changing from a super-Weyl invariant cutoff to a $\varphi$-independent one; we present this calculation in the Appendix C. In terms of the $\varphi$-dependence of the Wilsonian gauge couplings, the result is ${ }^{\S}$

$$
\tilde{f}_{a}^{W}(\varphi, \Phi)=f_{a}^{W}(\Phi)+\frac{3 \mathbf{c}_{a}}{8 \pi^{2}} \log \varphi
$$

where

$$
\mathbf{c}_{a}=\sum_{r} n_{r} T_{a}(r)-T\left(G_{a}\right)
$$

Here the sum is over representations $r$ of the gauge group $G, n_{r}$ is the number of multiplets of $Q^{I}$ transforming like $r, T_{a}(r)=\operatorname{Tr}_{r}\left(T_{(a)}^{2}\right)$ for $T_{(a)} \in G_{a}$ and $T\left(G_{a}\right)$ stands for $T_{a}$ (adjoint of $G_{a}$ ). After fixing the WZ gauge (2.18), the $\log \varphi$ terms in eqs. (2.20) translate into Kähler terms for the Wilsonian couplings of the ordinary gauge fields:

$$
\left(g_{a}^{W}\right)^{-2}(\Phi, \bar{\Phi} ; \mathrm{WZ}) \equiv \operatorname{Re} \tilde{f}_{a}^{W}=\operatorname{Re} f_{a}^{W}(\Phi)+\mathbf{c}_{a} \frac{\kappa^{2}}{16 \pi^{2}} K(\Phi, \bar{\Phi})
$$

which have a rather different dependence on the moduli fields than the classical couplings $g_{a}^{-2}=\operatorname{Re} f_{a}(\Phi)$. (Note that only the $O\left(M_{\mathrm{Pl}}^{2}\right)$ part of $K$ is relevant in this formula, so it does not pay to discriminate between the classical and the quantum Kähler functions or between $K_{\bmod }(\Phi, \bar{\Phi})$ and $K(\Phi, \bar{\Phi}, Q, \bar{Q})$.)

Technically, the origin of the $\log \varphi$ term in eq. (2.20) is in the unavoidable $\varphi$ dependence of the super-Weyl invariant cutoff. From the Wilsonian EQFT point of

\footnotetext{
$\S$ For matter-less supersymmetric Yang-Mills theories, a similar result was obtained in ref. [10] from a somewhat different point of view.
} 
view, this means that the super-Weyl invariance of the quantum theory is anomalous (there is no field independent cutoff that respects the super-Weyl symmetry), but it can be restored with the help of explicit local counter-terms such as the $\log \varphi$ term in $\tilde{f}_{a}^{W}$. In that sense the $\log \varphi$ can be viewed as a local Wess-Zumino counter-term cancelling the potential super-Weyl anomaly. Like other anomalies, the anomaly of the super-Weyl symmetry can be calculated in many ways, and one does not have to rely on the particular cutoff described in Appendix B to reproduce eq. (2.20). We conclude this section of the article with an alternative derivation of eq. (2.20) based upon the Adler-Bell-Jackiw anomaly of the axial symmetry of charged fermions.

The super-Weyl transformations (2.9) contain an R-symmetry, under which the scalar, the vector and the gravitational fields of the theory remain invariant, but the spinor fields and some of the auxiliary fields change their phases. Indeed, for $\tau$ that is purely bosonic, imaginary and constant, $\tau(z) \equiv i \nu$, the matter fermions and the gauginos transform like

$$
\Psi_{\alpha}^{I} \mapsto e^{+3 i \nu} \cdot \Psi_{\alpha}^{I}, \quad \lambda_{\alpha}^{(a)} \mapsto e^{-3 i \nu} \cdot \lambda_{\alpha}^{(a)}
$$

(cf. eqs. (2.11) and (2.10)). As far as the gauge fields are concerned, (2.23) is a classically-exact but anomalous axial symmetry. Moreover, this R-symmetry is spontaneously broken by $\langle\varphi\rangle \neq 0$, whose phase plays a role of a Goldstone boson, so its anomaly translates into anomalous effective couplings

$$
\sum_{a} \frac{-3 \mathbf{c}_{a}}{32 \pi^{2}} \arg (\varphi) \cdot\left(F_{m n} \tilde{F}^{m n}\right)_{a}
$$

of the phase of $\varphi$ to the gauge fields; note that the coefficients $\mathbf{c}_{a}$ of these couplings are exactly as in eq. (2.21). The couplings (2.24) are generated by the one-loop 
graphs
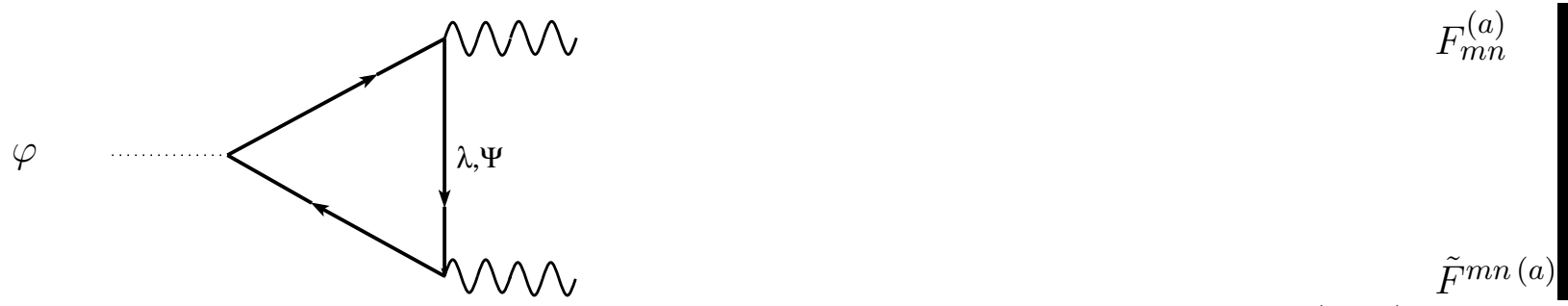

$(2.25)$

and are completely analogous to the anomalous effective coupling responsible for the $\pi^{0} \rightarrow \gamma \gamma$ decay in the standard model. However, unlike $\pi^{0}, \arg (\varphi)$ does not corresponds to any physical particle that can decay, propagate or have any interactions whatsoever. In fact, any physical coupling of the Weyl compensator would destroy the super-Weyl symmetry and prohibit the Weyl rescaling, leaving the theory with an irreparably non-Einsteinian gravity. To avoid such a calamity, the anomalous contribution of the triangle graphs (2.25) must be cancelled by an explicit counter-term in the Wilsonian action of the EQFT: instead of the original $\theta_{a}^{W}(\Phi, \bar{\Phi})$ - the moduli-dependent coefficients of the $\left(1 / 16 \pi^{2}\right) \operatorname{tr}_{a} F \tilde{F}$ operators — we should substitute

$$
\tilde{\theta}_{a}^{W}(\varphi, \bar{\varphi}, \Phi, \bar{\Phi})=\theta_{a}^{W}(\Phi, \bar{\Phi})-3 \mathbf{c}_{a} \arg (\varphi)
$$

Note that according to the Adler-Bardeen theorem, these counter-terms cancel not just the one-loop Feynman diagrams (2.25) but also all the higher-loop corrections to the anomalous couplings (2.24).

Formula (2.26) should hold in any background gauge invariant formulation of the theory, manifestly supersymmetric or not. However, given manifest SUSY, we can identify $\frac{-1}{8 \pi^{2}} \theta_{a}^{W}$ with the imaginary part of the supersymmetric gauge couplings $f_{a}^{W}$, which must be holomorphic functions of the chiral superfields; for $\tilde{f}_{a}^{W}$ this includes holomorphic dependence on the compensating superfield $\varphi$. Hence, there is only one way to obtain $\tilde{\theta}_{a}^{W}$ from a supersymmetric gauge coupling $\tilde{f}_{a}^{W}(\varphi, \Phi)$; the explicit form of such $\tilde{f}_{a}^{W}$ is given in eq. (2.20). 
Clearly, there is more to the super-Weyl symmetry (2.9) than just the axial symmetry (2.23), and in general, one should consider the anomalies of all the currents of (2.9). However, in a manifestly supersymmetric EQFT, all such currents are superpartners of each other and form a single supercurrent suffering from a single 'super-axial' anomaly. The easiest way to compute this anomaly and its effects is to notice that there is a unique gauge-invariant rigid-superfield expression whose bosonic part includes the coupling $(2.24)$ of $\operatorname{Im}(\varphi)$ to $F \tilde{F}$, namely

$$
\sum_{a} \frac{-3 \mathbf{c}_{a}}{32 \pi^{2}} \int d^{2} \Theta \log \varphi \cdot\left(W^{\alpha} W_{\alpha}\right)_{a}+\text { h. c.; }
$$

extending this expression to local SUSY is completely straightforward.

Before we discuss the relationship between the Wilsonian gauge couplings and the physical gauge couplings of an EQFT, let us summarize the important points of this section. We established that the moduli dependent Wilsonian couplings of a supersymmetric EQFT have to be defined with respect to a moduli independent cutoff $\Lambda$. Such a cutoff renders the super-Weyl symmetry anomalous. However, this super-Weyl anomaly can be cancelled by an explicit local Wess-Zumino counterterm, which introduces a compensator dependence into the Wilsonian gauge couplings as specified by eq. (2.20). The coefficient of the counter-term can be computed rather simply by utilizing the axial $U(1)$ part of the super-Weyl symmetry which re-phases the fermions of the theory. 


\section{Physical Gauge Couplings and the Perturbative Effects of the Super-Weyl Anomaly}

\subsection{Effective Gauge Couplings in Massless EQFTs.}

The Wilsonian couplings we have discussed so far are the parameters of a local Wilsonian action of an EQFT, from which the high-energy degrees of freedom have been integrated out, but the relevant low-energy degrees of freedom are still present in the matter and gauge superfields $Q^{I}, \bar{Q}^{\bar{I}}$ and $V^{(a)}$. In this section, we turn our attention to the physical effective couplings - the parameters of the S-matrix or of the closely-related generating functional, which results from integrating out all the quantum degrees of freedom, both high-energy and low-energy. (Perturbatively, the generating functional is the sum of all 1PI Feynman graphs). For example, given a background-covariant generating functional, the easiest definition of the effective gauge coupling $\left\{g_{a}\right\}^{\star}$ is via the two-point function

$$
\mathcal{A}\left(A_{m}^{(a)}(-p), A_{n}^{(a)}(p)\right)=\left(p^{2} g_{m n}-p_{m} p_{n}\right) \frac{1}{\left\{g_{a}(p)\right\}^{2}}
$$

for two un-renormalized gauge fields belonging to $G_{a}$. Generally, the effective couplings are momentum dependent (and thus are often called the "running effective couplings"), and this dependence is often non-local, i.e., becomes singular when some of the relevant momenta approach zero. Apart from possible thresholds, the dependence of the effective couplings on the overall momentum scale $p$ is logarithmic and governed by the Callan-Symanzik equations, such as

$$
\frac{\partial\left\{g_{a}(p)\right\}}{\partial \log p}=\beta_{a}(\{g\}, \ldots)=\frac{\mathbf{b}_{a}\left\{g_{a}\right\}^{3}}{16 \pi^{2}}+\frac{\mathbf{b}_{a}^{(2)}\left\{g_{a}\right\}^{5}}{256 \pi^{4}}+\cdots
$$

\footnotetext{
* Following the notations of ref. [8], we use the curly braces ' \{\} ' to distinguish the effective couplings of a quantum theory from its Wilsonian couplings or their classical counterparts. In ref. [1], the square brackets '[ ]' were used for the same purpose.
} 
Here

$$
\mathbf{b}_{a}=\sum_{r} n_{r} T_{a}(r)-3 T\left(G_{a}\right)
$$

whereas the expressions for the $\mathbf{b}_{a}^{(2)}$ are more complicated and also depend on the Yukawa couplings $\left\{Y_{I J K}\right\}$. (The higher-order terms also depend on the choice of the renormalization scheme.)

Renormalization of the effective gauge couplings of a rigidly supersymmetric EQFT was studied by Novikov, Shifman, Vainshtein and Zakharov, ${ }^{[34,1]}$ who found exact relations between $\beta_{a}$ and the wave-function renormalization of the charged matter fields $Q^{I}$. These relations are integrable and lead to

$$
\begin{aligned}
F_{a} \equiv\left\{g_{a}(p)\right\}^{-2}-\frac{T\left(G_{a}\right)}{8 \pi^{2}} \log \left\{g_{a}(p)\right\}^{-2} & \\
& +\sum_{r} \frac{T_{a}(r)}{8 \pi^{2}} \log \operatorname{det}\left\{Z^{(r)}(p)\right\}+\frac{\mathbf{b}_{a}}{8 \pi^{2}} \log p
\end{aligned}
$$

being exact integrals of the renormalization group, $\frac{d F_{a}}{d p}=0$; the $\left\{Z^{(r)}(p)\right\}$ matrix here is the diagonal block of the renormalized kinetic-energy matrix $\left\{Z_{\bar{I} J}(p)\right\}$ for the matter fields $\bar{Q}^{\bar{I}}, Q^{J}$ that transform like $r$ under $G$. The integrals $F_{a}$ of the Callan-Symanzik equations for the effective gauge couplings are related to the invariants of the Wilsonian renormalization group for $f_{a}^{W}$, and because the Wilsonian supersymmetric gauge couplings do not renormalize beyond one loop, the relation reads simply

$$
F_{a}=\operatorname{Re} f_{a}^{W}+\frac{\mathbf{b}_{a}}{8 \pi^{2}} \log \Lambda
$$

$\Lambda$ being the ultraviolet cut-off scale. Note that the effective gauge couplings $\{g(p)\}$ generally do renormalize to all orders, including the two-loop order responsible for the $\mathbf{b}_{a}^{(2)}\{g\}^{5}$ terms in eqs. (3.2); correspondingly, eqs. (3.4) have logarithmic singularities as $\left\{g_{a}\right\} \rightarrow 0$. 
Eqs. (3.4) and (3.5) can be used to relate the dependence of $\left\{g_{a}(p ;\langle\Phi\rangle,\langle\bar{\Phi}\rangle)\right\}$ on the moduli VEVs to the moduli-dependence of the Wilsonian couplings $f_{a}^{W}(\Phi)$. The UV cutoff scale $\Lambda$ is field-independent, and the holomorphicity of $f_{a}^{W}(\Phi)$ translates via eqs. (3.4) into a very specific non-harmonicity of the moduli-dependence of the effective gauge couplings, which is further related by SUSY to very specific non-integrability of the effective axionic couplings of the moduli scalars. ${ }^{[8,35]}$ The analysis of ref. [1] was limited to rigidly supersymmetric EQFTs; its extension to locally supersymmetric theories is the main subject of this section. ${ }^{\dagger}$

The first step towards such an extension is to notice that as far as the renormalizable interactions of a low-energy EQFT are concerned, there is no difference between local and rigid supersymmetries. Hence, as long as the spacetime background of a locally supersymmetric EQFT is flat, the Callan-Symanzik equations for the relevant couplings of the theory are identical to those of its rigidly supersymmetric counterpart. In particular, eqs. (3.4) hold verbatim $-F_{a}$ are exact integrals of the renormalization group, i.e., do not depend on the momentum scale $p$.

Extending eqs. (3.5) to locally supersymmetric theories is more tricky: relations involving both physical and Wilsonian couplings would carry over only if the background of a locally supersymmetric theory is both physically and formally compatible with rigid SUSY. In other words, we need both the physical spacetime metric $g_{m n}$ and the superspace's vielbein $E_{M}^{A}$ to be flat at the same time:

$$
g_{m n}(x)=\eta_{m n} \quad \text { and } \quad E_{M}^{A}(x, \Theta, \bar{\Theta})=E_{M}^{A}[\text { flat }](\Theta, \bar{\Theta}) .
$$

Otherwise the relation between the Wilsonian couplings of the globally supersymmetric and the locally supersymmetric theory are modified by the super-Weyl

$\dagger$ Non-harmonic gauge couplings were first encountered in string theory in ref. [8] and further expanded upon in refs. [36-38]. They also appear in the context of the linear multiplet; this aspect is discussed in refs. $[10,11,27,39]$. A preliminary version of this article was presented in ref. [9]. 
anomaly. In the compensator formalism, the two conditions (3.6) are compatible only in the Wess-Zumino gauge (2.18) for the super-Weyl symmetry. Hence,

$$
\begin{aligned}
F_{a}(\Phi, \bar{\Phi}) & =\left(g_{a}^{W}\right)^{-2}(\Phi, \bar{\Phi} ; \mathrm{WZ})+\frac{\mathbf{b}_{a}}{8 \pi^{2}} \log \Lambda \\
& =\operatorname{Re} f_{a}^{W}(\Phi)+\frac{\mathbf{b}_{a}}{8 \pi^{2}} \log \Lambda+\mathbf{c}_{a} \frac{\kappa^{2}}{16 \pi^{2}} K(\Phi, \bar{\Phi})
\end{aligned}
$$

(cf. eq. (2.22)) or, in terms of the super-Weyl covariant Wilsonian couplings (2.20) and (2.13),

$$
F_{a}=\operatorname{Re} \tilde{f}_{a}^{W}+\frac{\mathbf{b}_{a}}{8 \pi^{2}} \log \Lambda+\mathbf{c}_{a} \frac{\kappa^{2}}{16 \pi^{2}} \widetilde{K}
$$

(the $\varphi$-dependent terms of the super-Weyl invariant couplings $\tilde{f}_{a}^{W}$ and $\widetilde{K}$ cancel in this equation).

The argument we have just used to derive eqs. (3.7) and (3.8) is rather heuristic and may appear to the reader as not too trustworthy. To eliminate all doubts, consider the origin of possible supergravitational corrections to the rigid-SUSY formula (3.5). The only source of these corrections is in the anomalies of the super-Weyl rescaling we discussed in the last section, and like the ordinary axial anomalies, these are exhausted at the one-loop level. Therefore, what we need to do is to calculate the effective gauge couplings $\left\{g_{a}(p)\right\}$ to the one-loop order and to interpret the result as the relation between $F_{a}$ and the Wilsonian gauge couplings $f_{a}^{W}$. If this one-loop calculation agrees with eqs. (3.7) and (3.8), then they automatically hold true to all orders of the perturbation theory and no further verification of their validity would be necessary.

The most direct way of calculating $\left\{g_{a}(p)\right\}$ in terms of the manifestly supersymmetric Wilsonian couplings of the theory is to provide the theory with a supersymmetric, etc., ultraviolet cutoff and then use superfield Feynman rules; this calculation is presented in the Appendix C. Alternatively, similar to the derivation of eq. (2.27) from the axial anomaly of the R-symmetry, we can relate the moduli 
dependence of $\left\{g_{a}\right\}$ to the effective axionic couplings of the moduli scalars to the CP-odd combinations of the gauge bosons. At the one-loop level, those effective axionic couplings can be easily computed using component-field Feynman rules and a background-covariant UV cutoff (however, there is no need for the cutoff to be supersymmetric, etc.). The rigid-SUSY analogue of this calculation is presented in ref. [35]; the locally supersymmetric case is discussed in refs. [9-11] and also presented in Appendix D of this article. This calculation of $\left\{g_{a}\right\}$ is less direct, but it is also less formalism dependent; needless to say, the results of both calculations confirm eqs. (3.7) and (3.8).

\subsection{Kähler Transformations and Global Symmetries.}

Describing non-gauge interactions of scalar superfields in terms of a separate real-analytic Kähler function $K(\Phi, \bar{\Phi}, Q, \bar{Q})$ and a separate complex-analytic superpotential $W(\Phi, Q)$ is somewhat redundant. Classically, the so-called Kähler transformations ${ }^{[4-6]}$

$$
\begin{aligned}
K(\Phi, \bar{\Phi}, Q, \bar{Q}) & \rightarrow K(\Phi, \bar{\Phi}, Q, \bar{Q})+\mathcal{J}(\Phi, Q)+\mathcal{J}^{*}(\bar{\Phi}, \bar{Q}) \\
W(\Phi, Q) & \rightarrow W(\Phi, Q) \cdot \exp \left(-\kappa^{2} \mathcal{J}(\Phi, Q)\right) \\
f_{a}(\Phi, Q) & \rightarrow f_{a}(\Phi, Q)
\end{aligned}
$$

leave all physical quantities invariant. In the compensator formalism, these transformations are accompanied by the $\varphi$ rescaling ${ }^{[29]}$

$$
\varphi \rightarrow \varphi \cdot \exp \left(\frac{1}{3} \kappa^{2} \mathcal{J}(\Phi, Q)\right)
$$

which renders the functions $\widetilde{K}, \widetilde{W}$ and $\tilde{f}_{a}$ defined in eqs. (2.13)-(2.15) - and thus the action - manifestly invariant at the superfield level. (Note that (3.12) is not a part of any super-Weyl transformation - the gravitational superfields are inert under Kähler transformations). The $\mathcal{J}$ in eqs. (3.9) through (3.12) is an arbitrary 
holomorphic function of the chiral superfields $\Phi^{i}$ and $Q^{I}$, and 'holomorphic' here really means complex-analytic and non-singular. Hence, according to eq. (3.10), the only Kähler-invariant features of the superpotential $W$ are the location of its zeros; a superpotential without zeros can be completely Kähler-transformed away into a harmonic term in $\mathcal{G} \equiv \kappa^{2} K+\log |W|^{2}$ (cf. ref. [4]). However, the superpotential of an effective field theory usually has continuous varieties of zeros (including $W(\Phi, Q=0)=0$ for all values of the moduli $\left.\Phi^{i}\right)$, so in spite of the Kähler invariance of the theory, we have to describe it in terms of separate $W$ and $K$, even classically.

For locally supersymmetric EQFTs there is an even better reason to treat $W$ and $K$ as separate functions, namely the Kähler anomaly of the Wilsonian gauge couplings $f_{a}^{W}$. This anomaly is fairly obvious in the compensator formalism: Combining the demand for Kähler-invariance of the action with eqs. (2.15) for the Wilsonian gauge couplings, we immediately see that the classical transformation law (3.11) cannot be maintained at the quantum level. Instead, eq. (3.11) has to be replaced with

$$
\tilde{f}_{a}^{W} \rightarrow \tilde{f}_{a}^{W} \Longleftrightarrow f_{a}^{W} \rightarrow f_{a}^{W}-\frac{\mathbf{c}_{a}}{8 \pi^{2}} \kappa^{2} \mathcal{J}
$$

It is worth noting that this new transformation law can also be inferred from eqs. (3.4) and (3.7). The effective gauge couplings $\left\{g_{a}(p)\right\}$ are physical quantities and thus remain invariant under all symmetries of an EQFT. The renormalized kinetic-energy matrices $\left\{Z_{\bar{I} J}(p)\right\}$ do not change unless the matter fields themselves are rescaled or mixed with each other. No such mixing or rescaling is involved in the Kähler transformations, so a quick glance upon the right hand side of eq. (3.4) tells us that $F_{a}$ are Kähler invariant. An equally quick glance at the right hand

\footnotetext{
* Not even implicitly, through a Weyl rescaling, because the physical super-Weyl gauge (2.18) is preserved by the combined effect of eqs. (3.9) and (3.12).
} 
side of eq. (3.7) then tells us that $f_{a}^{W}$ cannot possible be Kähler invariant but must instead transform in accordance with eq. (3.13).

By themselves, the Kähler transformations (3.9-10) do not constitute a physical symmetry of an EQFT since they leave all the physical fields unchanged. However, they often appear in the context of a global non-linear internal symmetry of an EQFT, which transforms the chiral superfields $\Phi^{i}, Q^{I}$ into some holomorphic functions of each other while leaving the superpotential and the Kähler function of the theory invariant modulo a Kähler transform, that is,

$$
\begin{aligned}
K\left(\Phi^{\prime}, \bar{\Phi}^{\prime}, Q^{\prime}, \bar{Q}^{\prime}\right) & =K(\Phi, \bar{\Phi}, Q, \bar{Q})+\mathcal{J}(\Phi, Q)+\mathcal{J}^{*}(\bar{\Phi}, \bar{Q}) \\
W\left(\Phi^{\prime}, Q^{\prime}\right) & =W(\Phi, Q) \cdot \exp \left(-\kappa^{2} \mathcal{J}(\Phi, Q)\right)
\end{aligned}
$$

for some holomorphic $\mathcal{J}(\Phi, Q)$. In addition, a true symmetry should also preserve the gauge charges of all chiral superfields (i.e., commute with the gauge group) and preserve the classical gauge couplings:

$$
f_{a}\left(\Phi^{\prime}, Q^{\prime}\right)=f_{a}(\Phi, Q)
$$

Again, eqs. (3.16) are subject to anomalous corrections. In fact, there are two anomalies at play here: the Kähler anomaly (3.13), and the ordinary Konishi anomaly (present in global supersymmetry) due to redefinition of the charged matter superfields. ${ }^{[12]}$ Both anomalies can be obtained from direct superfield calculations, but by far the easiest way of extracting their combined effect is through eqs. (3.4) and (3.7). For simplicity, consider a symmetry that is linear with respect

\footnotetext{
$\dagger$ In refs. [10,11], a different mechanism was proposed for cancelling the Kähler anomaly of the gauge couplings: Instead of the Wess-Zumino $\log \varphi$ terms in $\tilde{f}_{a}^{W}$, one can couple the gauge superfields to a linear multiplet with appropriate transformation properties. Generically, this mechanism needs a separate linear multiplet for each independent gauge coupling.
} 
to the charged matter fields, or at least becomes linear in the $|Q| \ll M_{\mathrm{Pl}}$ limit:

$$
Q^{I} \mapsto Q^{\prime I}=\Upsilon_{J}^{I}(\Phi) Q^{J}+O\left(Q^{2} / M_{\mathrm{Pl}}\right)
$$

The effect of such a symmetry on the two-point Green's functions for the matter fields is obvious: In terms of $\left\{Z_{\bar{I} J}(p ;\langle\Phi\rangle,\langle\bar{\Phi}\rangle)\right\}$, we have, in matrix notations,

$$
\left\{Z\left(p ; \Phi^{\prime}, \bar{\Phi}^{\prime}\right)\right\}=\left(\Upsilon^{\dagger}(\bar{\Phi})\right)^{-1} \cdot\{Z(p ; \Phi, \bar{\Phi})\} \cdot(\Upsilon(\Phi))^{-1}
$$

An exact symmetry of an EQFT should leave $\left\{g_{a}(p ;\langle\Phi\rangle,\langle\bar{\Phi}\rangle)\right\}$ invariant; hence, according to eq. (3.4), $F_{a}$ should transform according to

$$
F_{a}\left(\Phi^{\prime}, \bar{\Phi}^{\prime}\right)=F_{a}(\Phi, \bar{\Phi})-\sum_{r}^{\text {matter }} \frac{T_{a}(r)}{8 \pi^{2}} \log \left|\operatorname{det} \Upsilon^{(r)}(\Phi)\right|^{2}
$$

It remains to substitute eqs. (3.18) and (3.14) into eq. (3.7) and make use of the holomorphicity of $f_{a}^{W}(\Phi)$; this implies

$$
f_{a}^{W}\left(\Phi^{\prime}\right)=f_{a}^{W}(\Phi)-\frac{\mathbf{c}_{a}}{8 \pi^{2}} \kappa^{2} \mathcal{J}(\Phi)-\sum_{r}^{\text {matter }} \frac{T_{a}(r)}{4 \pi^{2}} \log \operatorname{det} \Upsilon^{(r)}(\Phi)
$$

The power of the anomalous symmetry relations (3.19) is that they impose analytic constraints on a holomorphic function $f^{W}(\Phi)$ relating its values at distant points in the moduli space. To illustrate the power of such constraints, consider what happens when the global symmetry group $\mathbf{S}$ is discrete but big enough to assure the compactness of $M_{0} / \mathbf{S}$ (we denote the moduli space by $M_{0}$ ). Classically, this immediately implies moduli-independence of all the supersymmetric gauge couplings $f_{a}$ : An S-invariant holomorphic function $f_{a}(\Phi)$ is the same as a single-valued holomorphic function on the compact complex manifold $M_{0} / \mathbf{S}$, and therefore has to be constant. In a quantum theory, the anomalous right hand side 
of eq. (3.19) guarantees that $f_{a}^{W}$ do depend non-trivially on the moduli fields; however, the exact form of such dependence is completely determined by the symmetry constraints. Indeed, were there two holomorphic functions $f_{1}(\Phi)$ and $f_{2}(\Phi)$ satisfying the same anomalous symmetry relation of the form (3.19), then the difference $f_{1}-f_{2}$ would be a holomorphic function that is invariant under all the symmetries. Note that although the above argument assumed that the moduli space becomes compact after symmetry-related points are identified with each other, the same result is obtained when such space is not quite compact, but some physical reasons limit the growth of $f(\Phi)$ along the non-compact directions. Thus, given a sufficiently big discrete symmetry group and some physical limits on $g_{a}^{-2}$, the exact analytic form of the moduli dependence of the Wilsonian gauge couplings can be completely determined from the anomalous symmetry relations (3.19), up to moduli-independent overall constants.

One can show ${ }^{[9,10,40,28]}$ that for many vacuum families of the heterotic string, symmetry relations (3.19) indeed completely determine the moduli dependence of the $f^{W}$, or at least its dependence on some of the moduli. For example, for orbifolds which have exact 'duality' symmetries, the Wilsonian gauge couplings $f_{a}^{W}$ depend on the toroidal moduli $T^{i}$ of the orbifold according to

$$
f_{a}^{W}(T)=\sum_{i} \frac{\alpha_{i}^{a}}{8 \pi^{2}} \log \eta\left(i T^{i}\right)+\text { const }
$$

where $\alpha_{i}^{a}$ are integer coefficients computable at the tree level of the string and the 'constant' may be a function of other moduli, but not of the $T^{i}$. All one needs to know to derive eq. (3.20) is the spectrum of the massless charged particles of the theory and their classical couplings to the moduli $T^{i}$ and no string loop calculations are necessary, although the result (including the coefficients $\alpha_{i}^{a}$ ) always agrees with that of the one-string-loop calculation of ref. [8]. 


\subsection{EQFT with a Threshold.}

In the previous discussion of the effective couplings we have assumed that all light charged particles are exactly massless. Now consider what happens when some gauge or matter particles do have small masses of the order $M_{I} \ll M_{\mathrm{Pl}}$. Clearly, in the energy-momentum range $M_{I} \ll p \ll M_{\mathrm{Pl}}$ we can describe all interactions in terms of a massless EQFT while for $p \ll M_{I}$ we can use another EQFT in which only the truly massless particles appear as quantum fields. Formally, the lowerenergy EQFT can be obtained from the higher-energy EQFT by integrating out the massive fields. The goal of this subsection is to show the effect of integrating out the massive modes for the gauge couplings of locally supersymmetric theories.

We presume that some part of the gauge symmetry is spontaneously broken at the threshold scale $M_{I}$ but the supersymmetry itself remains unbroken. Consider the renormalization of the effective gauge couplings $\left\{g_{a}^{\prime}(p)\right\}$ corresponding to simple factors $G_{a}^{\prime}$ of the group $G^{\prime}$ of the gauge symmetries that remain unbroken below the threshold. Modifying the Shifman-Vainshtein arguments in order to account for the $O\left(M_{I}\right)$ masses of some of the matter and gauge superfields, we find that the exact renormalization-group integrals of the massive theory are

$$
\begin{aligned}
F_{a}^{M} \equiv & \left\{g_{a}^{\prime}(p)\right\}^{-2}+\sum_{r^{\prime}} \frac{T_{a}\left(r^{\prime}\right)}{8 \pi^{2}}\left(\log \operatorname{det}\left\{Z^{\left(r^{\prime}\right)}(p)\right\}-n_{r^{\prime}}(\text { gauge }) \log \left\{g_{r^{\prime}}(p)\right\}^{-2}\right) \\
& +\sum_{r^{\prime}} \frac{T_{a}\left(r^{\prime}\right)}{8 \pi^{2}}\left(\operatorname{Tr} \log h\left(p, \mathcal{M}_{\text {matter }}^{\left(r^{\prime}\right)}\right)-3 \operatorname{Tr} \log h\left(p, \mathcal{M}_{\text {gauge }}^{\left(r^{\prime}\right)}\right)\right)
\end{aligned}
$$

where the sums are over the irreducible representations $r^{\prime}$ of the unbroken gauge symmetry $G^{\prime}, n_{r^{\prime}}$ (gauge) are the numbers of the $r^{\prime}$ multiplets formed by the gauge fields, massive or massless, $\left\{g_{r^{\prime}}\right\}$ are the gauge coupling associated with those gauge fields, $\mathcal{M}_{\text {matter }}^{\left(r^{\prime}\right)}$ and $\mathcal{M}_{\text {gauge }}^{\left(r^{\prime}\right)}$ are, respectively, the canonically-normalized mass matrices for the matter and gauge superfields transforming like $r^{\prime}$ under $G^{\prime}$ and $h(p, \mathcal{M})$ is a complicated homogeneous function of the momentum $p$ and the 
mass $\mathcal{M}$. Fortunately, $h$ is only complicated near the threshold $p \sim \mathcal{M}$; well-below the threshold $h(p \ll \mathcal{M}) \approx \mathcal{M}$ while well above the threshold $h(p \gg \mathcal{M}) \approx c p$, $c$ being a numerical constant of order 1 whose value depends on details of the renormalization scheme used to define the running gauge couplings $\left\{g_{a}^{\prime}(p)\right\}$. For the renormalization scheme $(3.1), c=e^{-1}$; nevertheless, for the sake of notational simplicity, we will henceforth put $c=1$; modifying all the formulæ in this section to accommodate $c \neq 1$ is completely straightforward.

For simplicity, let us assume that each $G_{a}^{\prime}$ is wholly contained in a single simple factor $G_{a}$ of the full gauge group $G$, although one can have the same $G_{a}$ for several $G_{a}^{\prime}$ (as in GUTs). For momenta $p$ so far above the threshold scale $M_{I}$ that the masses become negligible, the last term in eq. (3.21) reduces to $\frac{1}{8 \pi^{2}} \mathbf{b}_{a} \log p$. At the same time, we have $\left\{g_{r^{\prime}}\right\}=\left\{g_{a}\right\}$ for all gauge bosons belonging to $G_{a}$ (and for all others $T_{a}\left(r^{\prime}\right) \equiv 0$ ) while for the matter fields $\left\{Z^{\left(r^{\prime}\right)}\right\}=\left\{Z^{(r)}\right\}$ for all $r^{\prime}$ representing pieces of the same multiplet $r$ of $G$. Let us assemble all thus related terms in eq. (3.21) together; comparing the result with the massless eq. (3.4), we immediately see that

$$
F_{a}^{M}=F_{a}=\operatorname{Re} f_{a}^{W}+\frac{\mathbf{c}_{a} \kappa^{2}}{16 \pi^{2}} K+\frac{\mathbf{b}_{a}}{8 \pi^{2}} \log \Lambda
$$

the second equality here follows from eq. (3.7), which applies because masses do not affect the anomaly of super-Weyl transformations.

Now consider the low-energy limit of eq. (3.21). Segregating the contributions of the massless particles from those of particles with masses $O\left(M_{I}\right)$, we have

$$
F_{a}^{M}=F_{a}^{\prime}-\Delta F_{a}
$$

where

$$
\begin{aligned}
F_{a}^{\prime}= & \left\{g_{a}^{\prime}(p)\right\}^{-2}-\frac{T\left(G_{a}^{\prime}\right)}{8 \pi^{2}}\left(\log \left\{g_{a}^{\prime}(p)\right\}^{-2}+3 \log p\right) \\
& +\sum_{r^{\prime}} \frac{T_{a}\left(r^{\prime}\right)}{8 \pi^{2}}\left(\log \operatorname{det}\left\{Z^{\left(r^{\prime}\right)}(p)\right\}+n_{r^{\prime}} \log p\right)_{\text {massless matter }}
\end{aligned}
$$


and

$$
\begin{aligned}
\Delta F_{a}=\sum_{r^{\prime}} \frac{T_{a}\left(r^{\prime}\right)}{8 \pi^{2}}[ & \left(n_{r^{\prime}} \log \left\{g_{r^{\prime}}\right\}^{-2}+3 \log \operatorname{det}\left\{\mathcal{M}^{\left(r^{\prime}\right)}\right\}\right)_{\text {massive vectors }} \\
& \left.-\left(\log \operatorname{det}\left\{Z^{\left(r^{\prime}\right)}\right\}+\log \operatorname{det}\left\{\mathcal{M}^{\left(r^{\prime}\right)}\right\}\right)_{\text {massive matter }}\right] .
\end{aligned}
$$

Obviously, $F_{a}^{\prime}$ are the exact analogues of $F_{a}$ for the low-energy theory from which all massive particles have decoupled. Since the same low-energy phenomenology can be obtained from a locally supersymmetric EQFT that retains only the massless fields, and since all the results of sections 2 and 3.1 apply verbatim to such an EQFT, we conclude that its Wilsonian gauge couplings $f_{a}^{\prime W}$ are related to the $F_{a}^{\prime}$ via an exact analogue of eq. (3.7), namely

$$
F_{a}^{\prime}=\operatorname{Re} f_{a}^{\prime W}+\frac{\mathbf{c}_{a}^{\prime} \kappa^{2}}{16 \pi^{2}} K+\frac{\mathbf{b}_{a}^{\prime}}{8 \pi^{2}} \log \Lambda^{\prime}
$$

The coefficient $\mathbf{c}_{a}^{\prime}$ in this formula is computed similar to eq. (2.21), but counting only the massless fields and ditto for the coefficient $\mathbf{b}_{a}^{\prime}$; this is different from the $\mathbf{c}_{a}$ and $\mathbf{b}_{a}$ appearing in eq. (3.22), which account for all the charged fields of the higherenergy EQFT, regardless of whether they have $O\left(M_{I}\right)$ masses or not. We show momentarily that the difference between $\mathbf{c}_{a}^{\prime}$ and $\mathbf{c}_{a}$ is necessary for maintaining supersymmetry on both sides of the threshold.

It is clear from eqs. (3.22), (3.23) and (3.26) that the key to threshold corrections to the Wilsonian gauge couplings is provided by $\Delta F_{a}$, which depends only on the masses and other properties of the massive particles. Let us reorganize those particles into proper massive supermultiplets. The supersymmetric Higgs mechanism makes the massive vector supermultiplets from gauge and massless matter supermultiplets, which acquire a common mass $\mathcal{M}=\{g\}\left\{Z_{\text {Higgs }}\right\}^{1 / 2} \mid\langle$ Higgs $\rangle \mid$; in our notations, all indices are suppressed and 〈Higgs〉 is the un-normalized VEV of the Higgs field times the appropriate group-theoretical factor. All other charged 
massive particles belong to non-Higgs massive scalar supermultiplets and have their masses because of the $\frac{1}{2} M_{I J}(\Phi) Q^{I} Q^{J}$ terms in the superpotential $W(\Phi, Q)$ of the EQFT; the canonically-normalized mass-square matrix for these particles is $\mathcal{M}^{2}=e^{\kappa^{2} K}\{Z\}^{-1 / 2} M^{\dagger}\left(\{Z\}^{-1}\right)^{\top} M\{Z\}^{-1 / 2}$. Substituting these cumbersomelooking masses into an already cumbersome eq. (3.25) actually results in a great simplification of the latter: After some algebra, we arrive at

$$
\begin{aligned}
\Delta F_{a}=\sum_{r^{\prime}} \frac{T_{a}\left(r^{\prime}\right)}{8 \pi^{2}}\left(2 \sum_{\substack{\text { massive } \\
\text { vectors }}}^{\left(r^{\prime}\right)} \log \mid\langle\text { Higgs }\rangle \mid\right. & \\
& \left.-\left[\log \mid \operatorname{det} M^{\left(r^{\prime}\right) \mid}+\frac{1}{2} n_{r^{\prime}} \kappa^{2} K\right]_{\text {massive matter }}^{\text {non-Higgs }}\right) .
\end{aligned}
$$

The un-normalized mass matrices $M^{\left(r^{\prime}\right)}$ are holomorphic functions of the chiral moduli fields $\Phi^{i}$ and, as argued in ref. [35], properly defined Higgs VEVs $\langle$ Higgs $\rangle$ are also holomorphic; the only non-harmonic terms here are those containing the Kähler function $K$. These terms are absent from the rigid-SUSY analogue of eq. $(3.27)^{[35]}$; just as the Kähler terms in eq. (3.7), they are peculiar to local SUSY. In fact the two kinds of Kähler terms are closely related to each other: Combining eqs. (3.22), (3.23), (3.26) and (3.27) together and separating holomorphic terms from terms proportional to $K$, we see that the Wilsonian gauge couplings of locally supersymmetric EQFTs describing the two sides of the threshold are related to each other via

$$
\begin{aligned}
\Delta f_{a}^{W}(\Phi) & \equiv\left(f_{a}^{\prime W}(\Phi)+\frac{\mathbf{b}_{a}^{\prime}}{8 \pi^{2}} \log \frac{\Lambda^{\prime}}{M_{I}}\right)-\left(f_{a}^{W}(\Phi)+\frac{\mathbf{b}_{a}}{8 \pi^{2}} \log \frac{\Lambda}{M_{I}}\right) \\
& =\sum_{\left(r^{\prime}\right)} \frac{T_{a}\left(r^{\prime}\right)}{8 \pi^{2}}\left(2 \sum_{\substack{\text { massive } \\
\text { vectors }}}^{\left(r^{\prime}\right)} \log \frac{\langle\operatorname{Higgs}\rangle}{M_{I}}-\left.\log \operatorname{det} \frac{M^{\left(r^{\prime}\right)}}{M_{I}}\right|_{\substack{\text { non-Higgs } \\
\text { massive matter }}}\right)
\end{aligned}
$$

( $M_{I}$ here is the 'official' threshold scale). This formula is identical to its rigidSUSY counterpart ( $c f$. [35]), but in the locally supersymmetric case, we also need 
an exact agreement between the non-holomorphic Kähler terms: In terms of their coefficients, we must have

$$
\mathbf{c}_{a}-\mathbf{c}_{a}^{\prime}=\sum_{r^{\prime}} T_{a}\left(r^{\prime}\right) \cdot n_{r^{\prime}}\left(\text { massive, non-Higgs } Q^{I}\right)
$$

Were eq. (3.29) to fail, the higher-energy EQFT and the lower-energy EQFT would become inconsistent with each other, and no supersymmetric correction to eq. (3.28) could possibly repair this inconsistency. Fortunately, simple algebra shows that

$$
\begin{aligned}
& \sum_{r^{\prime}} T_{a}\left(r^{\prime}\right) \cdot n_{r^{\prime}}\left(\text { massive, non-Higgs } Q^{I}\right) \\
& =\sum_{r^{\prime}} T_{a}\left(r^{\prime}\right)\left(n_{r^{\prime}}\left(\text { massive } Q^{I}\right)-n_{r^{\prime}}(\text { massive vectors })\right) \\
& =\left(\sum_{r} T_{a}(r) n_{r}\left(\text { all } Q^{I}\right)-T\left(G_{a}\right)\right)-\left(\sum_{r^{\prime}} T_{a}\left(r^{\prime}\right) n_{r^{\prime}}\left(\text { massless } Q^{I}\right)-T\left(G_{a}^{\prime}\right)\right)
\end{aligned}
$$

which indeed equals to $\mathbf{c}_{a}-\mathbf{c}_{a}^{\prime}$ according to eq. (2.21) and to the spectra of the two EQFTs. Conversely, eqs. (3.29) and (3.30) can be used to justify the presence of the Kähler term in eq. (3.7), and also to derive its coefficient (2.21), without recourse to either the super-Weyl symmetry or to the axial anomaly and relying solely on the locally supersymmetric formulæ for the particle's masses.

Formula (3.28) for the threshold corrections to the Wilsonian gauge couplings is holomorphic and completely determined at the one-loop level of integrating out the heavy fields. Threshold corrections to the effective gauge couplings behave quite differently. To be precise, let us define $\Delta_{a}$ as the difference between the low-energy effective couplings $16 \pi^{2} /\left\{g_{a}^{\prime}(p)\right\}^{2}$, extrapolated up to $p=M_{I}$ using the Callan-Symanzik equations of the lower-energy effective theory in which particles with $O\left(M_{I}\right)$ masses do not appear, and between the appropriate higher-energy effective couplings $16 \pi^{2} /\left\{g_{a}(p)\right\}^{2}$, extrapolated down to $p=M_{I}$ using the CallanSymanzik equations of the higher-energy effective theory in which all the $O\left(M_{I}\right)$ 
masses are ignored. ${ }^{\star}$ Using appropriate versions of eq. (3.4) for both theories, we find

$$
\begin{aligned}
\Delta_{a}=16 \pi^{2} \Delta F_{a}+2[ & T\left(G_{a}^{\prime}\right) \log \left\{g_{a}^{\prime}\left(p \nearrow M_{I}\right)\right\}^{-2}-\mathbf{b}_{a}^{\prime} \log M_{I} \\
& \left.-\sum_{r^{\prime}} T_{a}\left(r^{\prime}\right) \log \operatorname{det}\left\{Z_{\text {massless } Q}^{\left(r^{\prime}\right)}\left(p \nearrow M_{I}\right)\right\}\right] \\
-2[ & T\left(G_{a}\right) \log \left\{g_{a}\left(p \searrow M_{I}\right)\right\}^{-2}-\mathbf{b}_{a} \log M_{I} \\
& -\sum_{r^{\prime}} T_{a}\left(r^{\prime}\right) \log \operatorname{det}\left\{Z_{\text {all } \left.\left.{ }^{\left(r^{\prime}\right)}\left(p \nearrow M_{I}\right)\right\}\right]}\right.
\end{aligned}
$$

where $\Delta F_{a}$ is as in eqs. (3.25) and (3.27). Formula (3.31) is true to all orders of perturbation theory, but its application to higher orders requires care in using the right renormalization scheme for each appearance of the wave-function normalization matrix $\{Z\}$. Fortunately, at the one-loop level analysis of $\Delta_{a}$, this subtlety can be safely ignored, which reduces eq. (3.31) to a much simpler formula

$$
\begin{gathered}
\Delta_{a}^{1-\text { loop }}=16 \pi^{2} \operatorname{Re} \Delta f_{a}^{W}-\left(\mathbf{c}_{a}-\mathbf{c}_{a}^{\prime}\right) \kappa^{2} K-2\left(T\left(G_{a}\right)-T\left(G_{a}^{\prime}\right)\right) \log \left\{g_{a}\left(M_{I}\right)\right\}^{-2} \\
+\sum_{r^{\prime}} 2 T_{a}\left(r^{\prime}\right) \log \operatorname{det}\left\{Z_{\text {massive }}^{\left(r^{\prime}\right)}\left(M_{I}\right)\right\}
\end{gathered}
$$

in full agreement with the old result ${ }^{[41,42]}$

$$
\Delta_{a}^{1-\text { loop }}=\sum_{r^{\prime}} T_{a}\left(r^{\prime}\right)\left(4 \operatorname{Tr}\left[\log \frac{\mathcal{M}}{M_{I}}\right]_{\text {massive }}^{(r)}-2 \operatorname{Tr}\left[\log \frac{\mathcal{M}}{M_{I}}\right]_{\substack{\text { non-Higgs } \\ \text { massive } Q^{I}}}^{(r)}\right)
$$

Note that the second, the third and the fourth terms on the right hand side of eq. (3.32) are non-harmonic functions of the moduli VEVs. This is similar to the

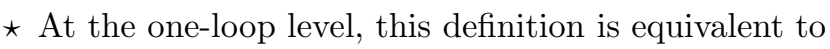

$$
\frac{16 \pi^{2}}{\left\{g_{a}^{\prime}\left(p^{\prime}<M_{I}\right)\right\}^{2}}=\frac{16 \pi^{2}}{\left\{g_{a}\left(p>M_{I}\right)\right\}^{2}}+2 b_{a} \log \frac{p}{M_{I}}+2 b_{a}^{\prime} \log \frac{M_{I}}{p^{\prime}}+\Delta_{a} ;
$$

at higher loop levels, one needs a much more complicated formula. 
non-harmonicity of the string-threshold corrections to the effective gauge couplings obtained in ref. [8].

We conclude this section by relaxing the assumption that each $G_{a}^{\prime}$ is wholly contained in a single $G_{a}$. Many unified models have intermediate-energy thresholds for which this assumption does not hold (e.g., the 'flipped' $S U(5) \otimes U(1)^{[43]}$ or the Oxford $S U(3)^{3}$ model $\left.^{[44]}\right)$ and the mapping between the low-energy gauge couplings $\left\{g_{a}^{\prime}\right\}$ and the high-energy gauge couplings $\left\{g_{a}\right\}$ is rather complicated. However, this complication is purely notational while the physics remains the same as in the case of single $G_{a}$ 's. In terms of the Wilsonian gauge couplings, we have

$$
f_{a}^{\prime W}(\Phi)+\frac{\mathbf{b}_{a}^{\prime}}{8 \pi^{2}} \log \frac{\Lambda^{\prime}}{M_{I}}=\sum_{b} C_{a b}\left(f_{b}^{W}(\Phi)+\frac{\mathbf{b}_{b}}{8 \pi^{2}} \log \frac{\Lambda}{M_{I}}\right)+\Delta f_{a}^{W}(\Phi),
$$

where $C_{a b}$ are group-theoretical factors characterizing the threshold and $\Delta f_{a}^{W}$ are exactly as in eq. (3.28). Note that since the Wilsonian gauge couplings renormalized only at one loop, eqs. (3.28) and (3.34) are exact, i.e., accurate to all orders of the perturbation theory.

\section{Gaugino Condensates and Effective Superpotentials}

In most theories of string unification, the four-dimensional EQFT describing very high energies has an unbroken gauge group that is a lot bigger than the $S U(3) \otimes S U(2) \otimes U(1)$ of the standard model. At some intermediate energy scale - well below $M_{\mathrm{Pl}}$ but well above the weak scale - the excess gauge symmetry decouples from the ordinary particles via spontaneous gauge symmetry breakdown, confinement or some combination of the two mechanisms. Implications of local SUSY for the spontaneous breakdown are discussed in section 3.3; the present section is devoted to a manifestly supersymmetric treatment of the confinement and associated dynamical effects: Formation of the gaugino condensate $\langle\lambda \lambda\rangle$ and breakdown of the perturbative degeneracy of the vacua with different moduli VEVs. 
The techniques we present here are purely field-theoretical and do not in any way depend on the string nature of the ultimate unification; all we presume is that the unified theory, whatever its nature, gives rise to a non-abelian asymptotically-free hidden gauge group $G_{a}$, which we shall henceforth denote as simply $G$.

Our discussion starts with locally supersymmetric Yang-Mills theories without charged matter fields. Pure SSYM theories have been extensively studied from different points of view; section 4.1 summarizes some generally known results and puts them in context of a locally supersymmetric pure Yang-Mills theory with a moduli-dependent gauge coupling. In section 4.2 we use EQFT techniques to calculate the effective superpotential for the moduli $W_{\bmod }(\Phi)$ that is induced by the gaugino condensation in a pure-SSYM hidden sector, and in section 4.3 we extend these techniques to hidden sectors with charged matter scalars. We show how to calculate $W_{\text {mod }}(\Phi)$ for any hidden sector, with or without charged scalars, as long as it has a stable, supersymmetric vacuum state. In section 4.4 we illustrate this technology on a few examples of hidden sectors one often encounters in stringunified theories. Finally, in section 4.5 we address the questions of the overall vacuum stability and of the supersymmetry breaking.

\subsection{Hidden Sectors Without Matter.}

By itself, gaugino condensation does not break supersymmetry. Indeed, as shown by E. Witten, ${ }^{[45]}$ in a rigidly supersymmetric theory of only gauge bosons and gauginos there is no spontaneous SUSY breakdown regardless of confinement, gaugino condensation and other non-perturbative phenomena. Implications of this result for locally supersymmetric Yang-Mills theories or for SSYM theories with moduli-dependent gauge couplings have been widely discussed in the literature. ${ }^{[13-23,9]}$ This section is the summary of the relevant results put together.

1. In rigid SUSY, the gaugino bilinear $\lambda^{\alpha} \lambda_{\alpha}$ is the lowest component of the composite gauge-invariant chiral superfield $U \equiv W^{\alpha} W_{\alpha}$. In local SUSY, 
there are two ways to generalize this superfield, namely ${ }^{[21]}$

$$
\mathcal{U} \equiv \mathcal{W}^{\alpha} \mathcal{W}_{\alpha} \quad \text { and } \quad \widehat{\mathcal{U}} \equiv \mathcal{U} / \varphi^{3}
$$

Both $\mathcal{U}$ and $\widehat{\mathcal{U}}$ are chiral and gauge invariant, but $\mathcal{U}$ is $\varphi$-independent and hence invariant under the Kähler transformations (3.9-11) while $\widehat{\mathcal{U}}$ is not; on the other hand, it is $\widehat{\mathcal{U}}$ and not $\mathcal{U}$ that is invariant under the super-Weyl transformations. The exact relation between these chiral superfields and the conventionally normalized gaugino bilinear is

$$
\lambda^{\alpha} \lambda_{\alpha}=\left.e^{\kappa^{2} \widetilde{K} / 2} \mathcal{U}\right|_{\Theta=\bar{\Theta}=0}=\left.(\bar{\varphi} / \varphi)^{3 / 2} e^{\kappa^{2} K / 2} \widehat{\mathcal{U}}\right|_{\Theta=\bar{\Theta}=0}
$$

By conventional normalization we mean that the gauginos $\lambda_{\alpha}^{(a)}$ have the same normalization as the gauge bosons $A_{m}^{(a)}$; this normalization is obtained after the Weyl rescaling, and indeed, in the Wess-Zumino gauge (2.18), eq. (4.2) reduces to $\lambda^{\alpha} \lambda_{\alpha}=\left.\mathcal{U}\right|_{\Theta=\bar{\Theta}=0}$.

2. The confinement scale $\mu$ of an asymptotically-free theory is the momentum scale below which perturbative renormalization of the running effective gauge coupling $\{g(p)\}$ no longer makes sense. (We presume $\mu \ll M_{\mathrm{Pl}}$.) For a supersymmetric Yang-Mills theory without charged scalars, eq. (3.4) breaks down at

$$
\begin{aligned}
\mu & =\left(8 \pi^{2} e / T(G)\right)^{1 / 3} p\{g(p)\}^{-2 / 3} \exp \left(-\frac{8 \pi^{2}}{3 T(G)\{g(p)\}^{2}}\right), \quad(\text { any } p \geq \mu) \\
& =\left(8 \pi^{2} e / T(G)\right)^{1 / 3} \Lambda \exp \left(-\frac{8 \pi^{2}}{3 T(G)} \operatorname{Re} f^{W}+\frac{1}{6} \kappa^{2} K\right),
\end{aligned}
$$

where the second equation follows from (3.7). Note that the first equation here has a pre-exponential factor $\{g\}^{-2 / 3}$ while in the second equation the Wilsonian gauge coupling $f^{W}$ appears only in the exponential — this reflects 
the fact that the effective gauge coupling is renormalized in all orders of perturbation theory, but the renormalization of the supersymmetric Wilsonian coupling $f^{W}$ stops at one loop. ${ }^{[3]}$ The Kähler term in the last exponential is peculiar to local SUSY; its coefficient does not depend on the size of the gauge group $G$.

3. Because of its relation (4.2) to the lowest component of a chiral superfield, the gaugino-bilinear operator $\lambda \lambda$ receives no anomalous corrections to its canonical scaling dimension 3. Consequently, $|\langle\lambda \lambda\rangle| \propto \mu^{3}$, with a couplingindependent proportionality coefficient of order 1 . At the same time, the phase of the gaugino condensate equals to the Wilsonian $\theta$-angle for $G$, divided by $T(G)$. With the help of eqs. (4.3) and (4.2) we can combine these two results into a single exact formula for the VEV of the composite superfield $\mathcal{U}$ in terms of the Wilsonian supersymmetric gauge coupling:

$$
\langle\mathcal{U}\rangle=\Lambda^{3} \exp \left(-\frac{8 \pi^{2}}{T(G)} \tilde{f}^{W}\right) \times[\text { an } O(1) \text { coupling-independent constant }] .
$$

This relation is holomorphic - the Kähler factors in eqs. (4.2) and (4.3) cancel each other, - which reflects the fact the SUSY is not spontaneously broken $\left(\mathcal{U}\right.$ and $\tilde{f}^{W}(\Phi, \varphi)$ are both composite chiral superfields). In terms of the super-Weyl invariant quantities, eq. (4.4) becomes

$$
\langle\widehat{\mathcal{U}}\rangle=\Lambda^{3} \exp \left(-\frac{8 \pi^{2}}{T(G)} f^{W}(\Phi)\right) \times \text { const } ;
$$

note that the Wess-Zumino $\log \varphi$ term in eq. (2.20) is absolutely necessary for the consistency of this formula, as is the fact that $\mathbf{c}=-T(G)$ for a matter-less Yang-Mills theory.

4. From the low-energy $(O(\mu))$ point of view, the $\widehat{\mathcal{U}}$ supermultiplet of the pure SSYM theory is analogous to the $\pi K \eta \eta^{\prime}$ meson nonet of the ordinary QCD - it describes the lightest composite particles of the confined theory, and 
its VEV serves as the order parameter of the chiral symmetry breakdown. In QCD, integrating out all hadronic degrees of freedom except for the order parameters leads to a sigma model; a similar treatment of the SSYM theory leads to an effective locally supersymmetric theory for $\widehat{\mathcal{U}}$. The vacuum structure of this effective theory is described by an effective superpotential $W_{\text {eff }}(\widehat{\mathcal{U}}, \Phi)$ where the $\operatorname{VEV}\langle\widehat{\mathcal{U}}\rangle$ is the SUSY-preserving solution of $\partial W_{\text {eff }} / \partial \widehat{\mathcal{U}}=0$. The specific formula (4.4) is reproduced by the effective superpotential $^{[16]}$

$$
W_{\text {eff }}(\widehat{\mathcal{U}}, \Phi)=\frac{1}{4} \widehat{\mathcal{U}} f^{W}(\Phi)+\frac{\widehat{\mathcal{U}}}{32 \pi^{2}}\left(T(G) \log \frac{\widehat{\mathcal{U}}}{\Lambda^{3}}+\text { const }\right)
$$

or, in terms of the Kähler-invariant superfield $\mathcal{U}^{[21]}$

$$
\begin{aligned}
\widetilde{W}_{\mathrm{eff}}(\mathcal{U}, \Phi, \varphi) & \equiv \varphi^{3} W_{\mathrm{eff}}(\mathcal{U}, \Phi) \\
& =\frac{1}{4} \mathcal{U} \tilde{f}^{W}(\Phi, \varphi)+\frac{\mathcal{U}}{32 \pi^{2}}\left(T(G) \log \frac{\mathcal{U}}{\Lambda^{3}}+\text { const }\right)
\end{aligned}
$$

The first term in this superpotential corresponds to the Wilsonian Lagrangian for the gauge superfields; the second term is a purely low-energy non-perturbative effect and thus involves only $\mathcal{U}$ itself but does not depend on the gauge coupling, the moduli or even the Weyl compensator $\varphi$.

5. There is an alternative argument for eqs. (4.6) and (4.7) for the effective superpotential that does not involve eq. (4.4). Instead, one assumes that a Kähler-invariant effective superpotential should look like $\widetilde{W}_{\text {eff }}=\frac{1}{4} \tilde{f}^{W} \mathcal{U}-$ $\Xi(\mathcal{U})$ where $-\Xi(\mathcal{U})$ is a 'dynamical' superpotential that does not depend on any superfields other than $\mathcal{U}$. The super-Weyl transformations should leave the $W_{\text {eff }} \equiv \widetilde{W}_{\text {eff }} / \varphi^{3}$ invariant; in light of eqs. (2.10) and (2.20), this means that $\Xi(\mathcal{U})$ should satisfy

$$
\Xi\left(e^{-6 \tau} \mathcal{U}\right)=e^{-6 \tau}\left[\Xi(\mathcal{U})-\frac{6 \mathbf{c} \tau}{32 \pi^{2}} \mathcal{U}\right]
$$

the solution to this constraint gives eq. (4.7) (for a SSYM theory without 
charged matter $\mathbf{c}=-T(G)$ ). For rigidly supersymmetric pure Yang-Mills hidden sectors, the same argument can be made in terms of the scale and $\mathrm{R}$-symmetries instead of super-Weyl; this is how the superpotential (4.6) was first derived in ref. [16].

Naturally, there are many other ways to obtain the effective superpotential. For example, for EQFTs based upon string orbifolds, the analytic form of the $W_{\text {eff }}$ can be deduced from the modular invariance. ${ }^{[1,23,9]}$

\subsection{Effective Potential for the Moduli.}

Classically, moduli VEVs $\left\langle\Phi^{i}\right\rangle$ parametrize a continuous family of exactly degenerate vacua of the unified theory. Because of the no-renormalization theorem for the superpotential (2.4), this exact degeneracy persists to all orders of perturbation theory. However, the non-perturbative effects associated with the confinement and with the formation of the gaugino condensate break this degeneracy, and once all of the strongly interacting fields are integrated out, the resulting effective theory acquires a non-trivial effective potential $\mathcal{V}_{\text {mod }}(\langle\Phi\rangle,\langle\bar{\Phi}\rangle)$ for the moduli fields.

Given the effective superpotential $W_{\text {eff }}(\widehat{\mathcal{U}}, \Phi)$, the calculation of the effective potential $\mathcal{V}_{\text {mod }}$ is rather simple: Starting with the formula (4.6) and the solution (4.5) for $\partial W_{\text {eff }}(\widehat{\mathcal{U}}, \Phi) / \partial \widehat{\mathcal{U}}=0$, one can integrate out the $\widehat{\mathcal{U}}$ superfield and derive an effective theory just for the moduli superfields. The superpotential of this effective theory is simply

$$
W_{\text {mod }}(\Phi) \equiv W_{\text {eff }}(\langle\widehat{\mathcal{U}}\rangle(\Phi), \Phi)=-\frac{T(G)}{32 \pi^{2}}\langle\widehat{\mathcal{U}}\rangle(\Phi)
$$

A similar formula describes the contribution of the hidden matter fields to the effective Kähler function for the moduli. However, this contribution is of the order $O\left(\mu^{2}\right)$ and thus negligible compared to the perturbative $K_{\bmod }=O\left(M_{\mathrm{Pl}}^{2}\right)$; for all practical purposes, the Kähler function of the effective theory is simply $K_{\bmod }(\Phi, \bar{\Phi})$. 
Together, $W_{\text {mod }}(\Phi)$ and $K_{\text {mod }}(\Phi, \bar{\Phi})$ yield an effective scalar potential according to the usual (classical) rules of the local SUSY ${ }^{[4-6]}$ :

$$
\begin{gathered}
\mathcal{V}_{\text {mod }}(\Phi, \bar{\Phi})=\left[G^{\bar{i} j}\left(\frac{\partial W_{\text {mod }}^{*}}{\partial \bar{\Phi}^{\bar{\imath}}}+\kappa^{2} \frac{\partial K_{\bmod }}{\partial \bar{\Phi}^{\bar{\imath}}} W_{\bmod }^{*}\right)\left(\frac{\partial W_{\bmod }}{\partial \Phi^{j}}+\kappa^{2} \frac{\partial K_{\text {mod }}}{\partial \Phi^{j}} W_{\text {mod }}\right)\right. \\
\left.-3 \kappa^{2}\left|W_{\text {mod }}\right|^{2}\right] \times e^{\kappa^{2} K_{\text {mod }}} .
\end{gathered}
$$

This potential must be invariant under all the exact symmetries of the EQFT and often this fact can be used to constrain the analytic form of the $W_{\bmod }(\Phi)$, for example in EQFTs based upon string orbifolds with duality symmetries. ${ }^{[20,22,46,9]}$

In this section we follow a different approach (pioneered by Ferrara, Girardello and Nilles ${ }^{[15]}$ and derive the effective scalar potential starting from a componentfield Wilsonian action for the gauge fields, the moduli and their superpartners. Using the most general rules of the quantum field theory, one can integrate out the strongly interacting fields and thus obtain $\mathcal{V}_{\bmod }(\Phi, \bar{\Phi})$ without ever using an effective superpotential. ${ }^{\star}$ However, as long as the strong interactions do not break local SUSY, either explicitly or spontaneously, the result must have the form (4.10) for some $W_{\bmod }(\Phi)$; in other words, the existence of an effective superpotential $W_{\text {mod }}(\Phi)$ is inevitable, and the only question is its exact form, which may agree or disagree with eqs. (4.6) and (4.9).

In this subsection we prove that the $W_{\text {mod }}$ induced by a pure-SSYM hidden sector indeed agrees with (4.6) and (4.9). Moreover, our arguments can be extended to any hidden sector, with or without matter fields, that has a stable supersymmetric vacuum. This extension is presented in the following section 4.3; for the present section we limit our attention to pure SSYM theories coupled to supergravity and to moduli fields.

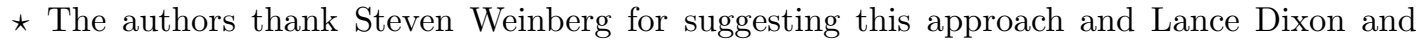
Michael Peskin for collaboration at an early stage of this calculation.
} 
In order to keep our notations simple, let $\chi$ denote all strongly interacting component fields, which for the theory at hand are simply the components of the Yang-Mills supermultiplet $\left(A_{m}^{(a)}, \lambda_{\alpha}^{(a)}, \bar{\lambda}_{\dot{\alpha}}^{(a)}, D^{(a)}\right)$ of the hidden sector $G$, plus ghosts due to the quantum gauge fixing. Similarly, let $\phi$ denote all the weakly interacting component fields, namely the moduli, the gravitational fields and all their superpartners, including the auxiliary fields as well as the components of the compensator $\varphi$. In the same notations, the Lagrangian can be written as

$$
\mathcal{L}=\mathcal{L}_{\mathrm{SSYM}}(\chi ; \phi)+\mathcal{L}_{\phi}^{0}(\phi)
$$

where the first term is the SSYM Wilsonian Lagrangian for the $\chi$ fields in a background of $\phi$ fields and the second term is the Lagrangian for the $\phi$ fields themselves. Ideally, the $\mathcal{L}_{\phi}^{0}$ should also be a Wilsonian Lagrangian, but because the interactions between quantum $\phi$ fields are badly non-renormalizable, we would not know how to regulate the resulting theory. Instead, we take $\mathcal{L}_{\phi}^{0}$ to be the "effective classical Lagrangian" i.e., the generating functional of an effective theory that consists only of the $\phi$ fields; formally, this generating functional is the sum of all 1PI Feynman graphs involving only $\phi$, but practically it should be calculated directly from the perturbative string theory.

Let us formally integrate out the strongly-interacting $\chi$ fields; this gives us an effective Lagrangian for the $\phi$ fields,

$$
\mathcal{L}^{\mathrm{eff}}(\phi)=\mathcal{L}_{\phi}^{0}(\phi)+\mathcal{L}_{\phi}^{1}(\phi)
$$

where

$$
\mathcal{L}_{\phi}^{1}=\left\langle\mathcal{L}_{\mathrm{SSYM}}\right\rangle+\left\langle\frac{\delta \mathcal{L}_{\mathrm{SSYM}}}{\delta \phi}\right\rangle(\phi-\langle\phi\rangle)+\cdots
$$

is the effective Lagrangian for $\phi$ induced by the strong interactions; below the confinement scale, this effective Lagrangian is local. The Feynman rules of the 
EQFT defined by eq. (4.12) are as follows: The propagators and the vertices are given by expanding both $\mathcal{L}_{\phi}^{0}$ and $\mathcal{L}_{\phi}^{1}$ into powers of $(\phi-\langle\phi\rangle)$; diagrammatically, we have:

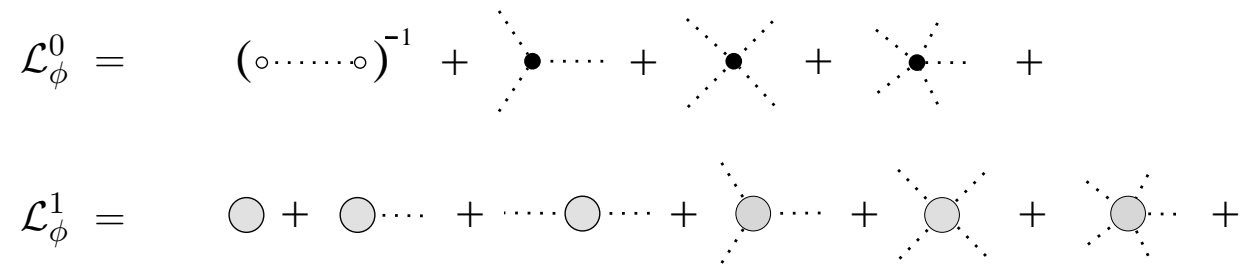

where the dotted lines are the perturbative propagators of $(\phi-\langle\phi\rangle)$, the solid circles are their perturbative vertices, and the bubbles

are the non-perturbative vertices induced by the strong interactions. Since $\mathcal{L}_{\phi}^{0}$ is a generating functional and not a Wilsonian Lagrangian, the only Feynman diagrams we should consider are those in which all loops contain at least one induced vertex

the loops involving only the classical vertices are already included in $\mathcal{L}_{\phi}^{0}$. Using the $\mathcal{L}^{0}$ rather than the full $\mathcal{L}^{\text {eff }}$ to define the propagators means that the induced vertices can have any number of external legs, $n=0,1,2,3, \ldots$ (as opposed to the perturbative vertices that always have $n \geq 3$ external legs). On the other hand, this makes for easy counting of the powers of the gravitational coupling $\kappa^{2}=8 \pi / M_{\mathrm{Pl}}^{2}$ : The propagators are proportional to $\kappa^{2}$, the perturbative vertices to $\kappa^{-2}$, and the induced vertices to $\kappa^{0}$; this reflects the facts that the entire $\mathcal{L}_{\phi}^{0}$ is proportional to $M_{\mathrm{Pl}}^{2}$ while the induced Lagrangian $\mathcal{L}_{\phi}^{1}$ is $M_{\mathrm{Pl}^{-}}$ independent. Moreover, because all loops have to include an induced vertex, they are effectively cut off at the confinement scale $\mu$ of the SSYM theory; consequently, the loops and the associated momentum integrals carry no extra powers of $M_{\mathrm{Pl}}$.

In any perturbative EQFT without a classical potential, the effective potential is the sum of all connected Feynman graphs with no external legs. For the problem 
at hand, the $\mathcal{L}_{\phi}^{0}$ contains no potential, and so, expanding in powers of $\kappa \mu$, we have

$\mathcal{V}_{\text {eff }}(\Phi, \bar{\Phi})=$

Naively, the first diagram here contributes an $O\left(\mu^{4}\right)$ term to the effective potential while the contributions of the other two diagrams are $O\left(\kappa^{2} \mu^{6}\right)$; the '...' stand for terms of still higher order in $(\kappa \mu)^{2}$. The first diagram corresponds to the effective potential generated by the SSYM theory, which vanishes since there is no spontaneous SUSY breaking in pure $\mathrm{SSYM}^{[45]}$; hence, the whole $\mathcal{V}^{\text {eff }}$ is at most of the order $O\left(\kappa^{2} \mu^{6}\right)$.

The second diagram in (4.15) gives us

$$
\int \frac{d^{4} p}{(2 \pi)^{4}} \operatorname{Str}\left[\Pi_{\phi_{1} \phi_{2}}(p) \times\left\langle\frac{\delta \mathcal{L}_{\mathrm{SSYM}}}{\delta \phi_{1}(p)} \frac{\delta \mathcal{L}_{\mathrm{SSYM}}}{\delta \phi_{2}(-p)}\right\rangle_{\mathrm{SSYM}}\right]
$$

where the supertrace is taken over all the weakly interacting component fields $\phi$, $\Pi_{\phi_{1} \phi_{2}}$ is the classical propagator matrix for those fields, and $\langle\cdots\rangle_{\mathrm{SSYM}}$ is the expectation value of the appropriate operator in the non-perturbative vacuum of the strongly interacting SSYM theory. In the absence of spontaneous SUSY breakdown in the strongly interacting sector, this $\langle\cdots\rangle_{\mathrm{SSYM}}$ is supersymmetric, and as long as the auxiliary fields are included among $\phi_{1}, \phi_{2}$, this supersymmetry holds even for the off-shell momenta $p$. Since the propagator matrix $\Pi$ is also supersymmetric, the supertrace in eq. (4.16) vanishes - the contributions of the bosonic and the fermionic components of any complete off-shell supermultiplet exactly cancel each other. Thus, unbroken supersymmetry of the SSYM theory also implies the vanishing of the second diagram in (4.15).

The third diagram in (4.15) yields

$$
\sum_{\phi_{1} \phi_{2}} \Pi_{\phi_{1} \phi_{2}}(p=0)\left\langle\frac{\delta \mathcal{L}_{\mathrm{SSYM}}}{\delta \phi_{1}(p=0)}\right\rangle\left\langle\frac{\delta \mathcal{L}_{\mathrm{SSYM}}}{\delta \phi_{2}(p=0)}\right\rangle
$$


Clearly, only the spinless fields $\phi_{1}, \phi_{2}$ contribute to this sum; these include the moduli scalars $\Phi^{i}$, the Weyl compensator $\varphi$, their auxiliary superpartners, and also the spinless auxiliary member $M$ of the supergravity multiplet. Moreover, the moduli scalars do not contribute in eq. (4.17) because the corresponding tadpoles vanish:

$\left\langle\frac{\delta \mathcal{L}_{\mathrm{SSYM}}}{\delta \Phi^{i}(p=0)}\right\rangle=\Phi^{i}$

(In a SSYM theory $\mathcal{V}_{\text {eff }}^{0}=$

vanishes regardless of the moduli VEVs.) A fortiori, there is no tadpole for the Weyl compensator $\varphi$. Thus the only contributions to eq. (4.17) come from the auxiliary fields, which is just as well since their propagators are momentumindependent and do not diverge at $p=0$.

Finally, two of the complex auxiliary fields, namely $M$ and $\left.F^{0} \equiv \log \varphi^{3}\right|_{\Theta \Theta}$, transform inhomogeneously under super-Weyl transformations, which allows us to fix or eliminate one of them by means of a suitable super-Weyl gauge conditions. For simplicity, we choose to set $M=0$ and leave $F^{0}$ unconstrained (however, the other components of the $\varphi$ superfield remain fixed by the truncated $(2.18)$ condition $\left.\left.\widetilde{K}\right|_{\text {no } \Theta}=\left.\widetilde{K}\right|_{\Theta}=\left.\widetilde{K}\right|_{\bar{\Theta}}=0\right)$. The propagator matrix for the $F^{0}$ and the $F^{i}=\left.\Phi^{i}\right|_{\Theta \Theta}$ is easily read from eqs. (2.1) and (2.13); hence, in light of $\mathcal{V}_{\text {eff }}^{0}=0$ and eq. (4.18), we have

$$
\begin{aligned}
\mathcal{V}^{\mathrm{eff}}=G^{\bar{\imath} j}\left(\left\langle\frac{\partial \mathcal{L}_{\mathrm{SSYM}}}{\partial F^{* \bar{\imath}}}\right\rangle+\kappa^{2} \frac{\partial K_{\mathrm{mod}}}{\partial \bar{\Phi}^{\bar{\imath}}}\left\langle\frac{\partial \mathcal{L}_{\mathrm{SSYM}}}{\partial F^{* 0}}\right\rangle\right) \times \\
\left(\left\langle\frac{\partial \mathcal{L}_{\mathrm{SSYM}}}{\partial F^{j}}\right\rangle+\kappa^{2} \frac{\partial K_{\bmod }}{\partial \Phi^{j}}\left\langle\frac{\partial \mathcal{L}_{\mathrm{SSYM}}}{\partial F^{0}}\right\rangle\right) \\
-3 \kappa^{2}\left|\left\langle\frac{\partial \mathcal{L}_{\mathrm{SSYM}}}{\partial F^{0}}\right\rangle\right|^{2}+O\left(\kappa^{4} \mu^{8}\right) .
\end{aligned}
$$

Note that the right hand side of this formula is a bilinear product of the operators' VEVs and not a VEV of the bilinear product of the same operators; for example, 
the gaugino condensate's contribution to the effective potential (4.19) is proportional to $|\langle\lambda \lambda\rangle|^{2}$ and not to $\langle\lambda \lambda \bar{\lambda} \bar{\lambda}\rangle^{\star}$. Therefore, eq. (4.19) may be re-written in terms of an effective superpotential if and only if the SSYM expectation values agree with

$$
\begin{aligned}
\left\langle\frac{\partial \mathcal{L}_{\mathrm{SSYM}}}{\partial F^{j}}\right\rangle(\Phi, \bar{\Phi}, \varphi, \bar{\varphi}) & =\frac{\partial \widetilde{W}_{\bmod }(\Phi, \varphi)}{\partial \Phi^{j}} \\
\text { and }\left\langle\frac{\partial \mathcal{L}_{\mathrm{SSYM}}}{\partial F^{0}}\right\rangle(\Phi, \bar{\Phi}, \varphi, \bar{\varphi}) & =\widetilde{W}_{\bmod }(\Phi, \varphi) \equiv \varphi^{3} W_{\bmod }(\Phi)
\end{aligned}
$$

for some holomorphic $W_{\bmod }(\Phi)$.

Up until this point we essentially followed the arguments of ref. [15] while using only the most general properties of a confining SSYM theory. Extracting the explicit component form of $\mathcal{L}_{\mathrm{SSYM}}$ from the relevant superspace integral in eq. (2.1), we have

$$
\frac{\partial \mathcal{L}_{\mathrm{SSYM}}}{\partial F^{j}}=\frac{\mathcal{U}}{4} \frac{\partial \tilde{f}^{W}(\Phi, \varphi)}{\partial \Phi^{i}} \text { and } \frac{\partial \mathcal{L}_{\mathrm{SSYM}}}{\partial F^{0}}=\frac{\mathcal{U}}{4} \frac{\partial \tilde{f}^{W}(\Phi, \varphi)}{\partial \log \varphi^{3}}
$$

which reduces eqs. (4.20) to

$$
\frac{\partial W_{\bmod }(\Phi)}{\partial \Phi^{i}}=\frac{\langle\widehat{\mathcal{U}}\rangle}{4} \frac{\partial \tilde{f}^{W}}{\partial \Phi^{i}} \quad \text { and } \quad W_{\bmod }(\Phi)=\frac{\langle\widehat{\mathcal{U}}\rangle}{4} \frac{\partial \tilde{f}^{W}}{\partial \log \varphi^{3}}
$$

Note that these equations would be inconsistent without the $\varphi$-dependent WessZumino term in the Wilsonian gauge coupling $\tilde{f}^{W}$ and therefore would fail to

* This has to do with the fact that the $F^{i}, F^{0}$ and $M$ auxiliary fields contribute to the effective potential via both the second and the third diagrams (4.15), but the contributions via the second diagrams cancel against those of the other components of the $\Phi^{i}, \varphi$ and gravitational supermultiplets. The distinction between the 'connected' parts of the VEVs such as $\langle\lambda \lambda \bar{\lambda} \bar{\lambda}\rangle$ and their 'disconnected' parts such as $\langle\lambda \lambda\rangle\langle\bar{\lambda} \bar{\lambda}\rangle$ corresponds to the distinction between the second and the third diagrams (4.15), both with an auxiliary propagator. It is important to keep track of this distinction; this is precisely why we did not integrate out the auxiliary superfields at the beginning of our calculations. 
produce an effective potential for the moduli that is consistent with eq. (4.10) and local SUSY; this is precisely the problem encountered in ref. [15]. On the other hand, given the correct Wilsonian coupling (2.20), eqs. (4.22) are consistent, but only if $\langle\widehat{\mathcal{U}}\rangle(\Phi)$ satisfies eq. (4.5) (note that $\mathbf{c}=-T(G)$ for a pure SSYM theory), and they have a unique solution for $W_{\bmod }(\Phi)$, namely eq. (4.9).

Usually, eq. (4.5) is derived in the way presented in section 4.1 - from the renormalization group equation for the gauge coupling, supplemented by the phase formula for the gaugino condensate. The moduli fields do not enter into this derivation, they are simply parameters that affect the Wilsonian gauge coupling $f^{W}(\Phi)$. Here we obtain exactly the same equation for $\langle\widehat{\mathcal{U}}\rangle$ from the argument that has nothing to do with the renormalization group and everything to do with the moduli: Eq. (4.5) must hold, or else the effective potential for the moduli would not be locally supersymmetric. We find it remarkable that the two totally unrelated arguments produce the same result. This agreement confirms that eq. (4.5) must be exact; there can be no corrections that are not suppressed by higher powers of $\mu / M_{\mathrm{Pl}}$. Moreover, the fact that the only solution to eqs. (4.22) is the same $W_{\bmod }(\Phi)$ that is obtained from eq. (4.6) by integrating out the $\widehat{\mathcal{U}}$ field gives us a much better confidence in the superpotential (4.6) than eq. (4.5) alone. Indeed, eq. (4.6) gives the only form for the $W_{\text {eff }}(\Phi, \widehat{\mathcal{U}})$ that lead to correct formulæ (4.5) and (4.9) for the $\langle\widehat{\mathcal{U}}\rangle(\Phi)$ and for the $W_{\bmod }(\Phi)$ and does so without any unnatural fine tuning of its parameters. In our opinion, this proves that the superpotential (4.6) is exact, modulo corrections suppressed by negative powers of $M_{\mathrm{Pl}}$.

\subsection{Supersymmetric Hidden Sectors with Matter.}

Calabi-Yau compactifications of the ten-dimensional heterotic string give rise to hidden sectors with $G \subset E_{8}^{\prime}$ and no matter multiplets charged under $G^{[47]}$ For more general four-dimensional string vacua, absence of the hidden matter is the exception rather than the rule. In order to understand what happens in string models of this kind, we now turn our attention to hidden sectors with charged 
matter. $^{[16-19,24]}$ In this section, we assume that the vacuum state of the hidden sector is stable and supersymmetric; these assumptions allow us to extend the analysis of section 4.2 to the more generic case at hand. Hidden sectors that break SUSY or have unstable vacua will be dealt with in section 4.5.

Let us recall the arguments we used to derive eqs. (4.19) and (4.20). In those arguments, we made no use of any features that are peculiar to matter-less SSYM theories but used only the general properties of their strong dynamics, namely the stability of the vacuum, the confinement and the unbroken supersymmetry. Hence, for any hidden sector with matter that has the same general properties, eqs. (4.19) and (4.20) should hold true. The only difference is that the $\mathcal{L}_{\mathrm{SSYM}}$ should be extended to $\mathcal{L}_{\text {hidden }}$ that also accommodates the hidden matter multiplets $Q^{I}$. Thus, instead of eqs. (4.21) we now have

$$
\begin{gathered}
\frac{\partial \mathcal{L}_{\text {hidden }}}{\partial F^{i}}=\frac{\mathcal{U}}{4} \frac{\partial \tilde{f}_{W}}{\partial \Phi^{i}}+\frac{\partial \widetilde{W}}{\partial \Phi^{i}}+\overline{F^{I}} Q^{J} \frac{\partial Z_{\bar{I} J}}{\partial \Phi^{i}} \\
\text { and } \frac{\partial \mathcal{L}_{\text {hidden }}}{\partial F^{0}}=\frac{\mathcal{U}}{4} \frac{\partial \tilde{f}_{W}}{\partial \log \varphi^{3}}+\frac{\partial \widetilde{W}}{\partial \log \varphi^{3}}+\overline{F^{I}} Q^{J} \frac{\partial Z_{\bar{I} J}}{\partial \log \varphi^{3}} .
\end{gathered}
$$

Fortunately, the unbroken supersymmetry of the hidden sector prevents the operators $\overline{F^{I}} Q^{J}$ from acquiring non-zero expectation values, so when we substitute eqs. (4.23) into eqs. (4.20) for the effective superpotential, we have only

$$
\frac{\partial W_{\text {mod }}(\Phi)}{\partial \Phi^{i}}=\frac{1}{4} \frac{\partial f_{W}}{\partial \Phi^{i}}\langle\widehat{\mathcal{U}}\rangle+\left\langle\frac{\partial W(Q, \Phi)}{\partial \Phi^{i}}\right\rangle
$$

and

$$
W_{\bmod }(\Phi)=\frac{\mathbf{c}}{32 \pi^{2}}\langle\widehat{\mathcal{U}}\rangle+\langle W(Q, \Phi)\rangle
$$


to worry about. The precise meaning of the $\left\langle\partial W / \partial \Phi^{i}\right\rangle$ here is

$$
\begin{aligned}
\left\langle\frac{\partial W(Q, \Phi)}{\partial \Phi^{i}}\right\rangle & =\frac{1}{2} \frac{\partial M_{I J}}{\partial \Phi^{i}}\left\langle Q^{I} Q^{J}\right\rangle_{\mathrm{hid}}+\frac{1}{3} \frac{\partial Y_{I J K}}{\partial \Phi^{i}}\left\langle Q^{I} Q^{J} Q^{K}\right\rangle_{\mathrm{hid}}+\cdots \\
& \equiv \sum_{t} \frac{\partial y_{t}(\Phi)}{\partial \Phi^{i}}\left\langle\mathcal{Q}^{t}\right\rangle_{\mathrm{hid}}
\end{aligned}
$$

where the '...' correspond to the non-renormalizable terms in the superpotential that may become relevant because of unusually large VEVs of the hidden matter scalars $Q^{I}$. On the second line in (4.26), $\mathcal{Q}^{t}$ runs over all the relevant gauge-invariant polynomials of the hidden matter superfields $Q^{I}$ and the $y_{t}$ are the corresponding masses or couplings.

The operators $\widehat{\mathcal{U}}$ and $\mathcal{Q}^{t}$ are chiral and their expectation values in a supersymmetric vacuum depend on the holomorphic couplings of the hidden sector, namely the $f_{W}(\Phi)$ and the $y_{t}(\Phi)$, but not on the non-holomorphic couplings such as the $Z_{\bar{I} J}$ matrices. Furthermore, the functional form of $\langle\widehat{\mathcal{U}}\rangle\left(f_{W}, y\right)$ and $\left\langle\mathcal{Q}^{t}\right\rangle\left(f_{W}, y\right)^{\star}$ is strongly constrained by the eqs. (4.24). Indeed, once we write eqs. (4.24) in terms of $\langle\widehat{\mathcal{U}}\rangle\left(f_{W}(\Phi), y(\Phi)\right)$ and $\left\langle\mathcal{Q}^{t}\right\rangle\left(f_{W}(\Phi), y(\Phi)\right)$ it becomes obvious that they are mathematically consistent with each other if and only if

$$
\begin{aligned}
W_{\bmod }(\Phi) & =\mathbf{W}\left(f_{W}, y\right) \\
\frac{1}{4}\langle\widehat{\mathcal{U}}\rangle(\Phi) & =\frac{\partial \mathbf{W}\left(f_{W}, y\right)}{\partial f_{W}} \\
\left\langle\mathcal{Q}^{t}\right\rangle(\Phi) & =\left.\frac{\partial \mathbf{W}\left(f_{W}, y\right)}{\partial y_{t}}\right|_{f_{W}(\Phi), y(\Phi)}
\end{aligned}
$$

for some holomorphic function $\mathbf{W}$ of the gauge and the Yukawa couplings. The form of eqs. (4.27) immediately suggests a Legendre transform that replaces a holomorphic function $\mathbf{W}$ of the couplings $f_{W}$ and $y_{t}$ with a holomorphic function

\footnotetext{
$\star y$ without a subscript stands for the whole set of $y^{t}$.
} 
$\Xi$ of the condensates $\widehat{\mathcal{U}}$ and $\mathcal{Q}^{t}$ (but not of the $f_{W}$ or $y_{t}$ ). After the transform, all of the eqs. (4.27) can be expressed in terms of a single effective superpotential

$$
W_{\mathrm{eff}}(\Phi, \widehat{\mathcal{U}}, \mathcal{Q})=\frac{1}{4} f_{W}(\Phi) \widehat{\mathcal{U}}+\sum_{t} y_{t}(\Phi) \mathcal{Q}^{t}-\Xi(\widehat{\mathcal{U}}, \mathcal{Q})
$$

Specifically, the expectation values $\langle\widehat{\mathcal{U}}\rangle$ and $\left\langle\mathcal{Q}^{t}\right\rangle$ are the supersymmetric solutions of

$$
\begin{array}{r}
\frac{\partial W_{\mathrm{eff}}(\Phi, \widehat{\mathcal{U}}, \mathcal{Q})}{\partial \widehat{\mathcal{U}}}=\frac{\partial W_{\mathrm{eff}}(\Phi, \widehat{\mathcal{U}}, \mathcal{Q})}{\partial \mathcal{Q}^{t}}=0, \\
\text { i.e., } \quad \frac{\partial \Xi}{\partial \widehat{\mathcal{U}}}=\frac{1}{4} f_{W}(\Phi) \quad \text { and } \quad \frac{\partial \Xi}{\partial \mathcal{Q}^{t}}=y_{t}(\Phi),
\end{array}
$$

and the moduli superpotential is simply

$$
W_{\text {mod }}(\Phi)=W_{\text {eff }}(\Phi,\langle\widehat{\mathcal{U}}\rangle(\Phi),\langle\mathcal{Q}\rangle(\Phi))
$$

exactly as in eq. (4.9). Physically, the first two terms in the superpotential $W_{\text {eff }}$ have obvious origins at the tree level of the hidden sector, while the $-\Xi(\widehat{\mathcal{U}}, \mathcal{Q})$ term should be thought of as dynamically induced at the non-perturbative level. Notice that by construction, this dynamical term does not depend on any couplings of the hidden sector; its exact form, therefore, should be completely determined by the gauge group $G$ and the spectrum of the charged matter fields $Q^{I}$. It is this coupling-blindness that will allow us to derive exact formulæ for the $W_{\text {eff }}$ for many EQFTs.

In the previous section, we saw that $W_{\text {eff }}$ is determined by requiring the consistency of eqs. (4.22). Similarly, eqs. (4.25), (4.28) - (4.30) imply

$$
\frac{\partial \Xi(\widehat{\mathcal{U}}, \mathcal{Q})}{\partial \widehat{\mathcal{U}}}-\frac{\Xi(\widehat{\mathcal{U}}, \mathcal{Q})}{\mathcal{U}}=\frac{\mathbf{c}}{32 \pi^{2}}
$$

and since the $\Xi(\widehat{\mathcal{U}}, \mathcal{Q})$ is blind to the moduli and to the couplings, eq. (4.31) must be satisfied identically, i.e., for all possible values of $\widehat{\mathcal{U}}$ and $\mathcal{Q}^{t}$. Solving eq. (4.31) 
and substituting the solution into eq. (4.28) gives us

$$
W_{\text {eff }}(\Phi, \widehat{\mathcal{U}}, \mathcal{Q})=W_{\text {tree }}(\Phi, \mathcal{Q})+\frac{\widehat{\mathcal{U}}}{4} f_{W}(\Phi)-\frac{\widehat{\mathcal{U}}}{32 \pi^{2}}\left(\mathbf{c} \log \frac{\widehat{\mathcal{U}}}{\Lambda^{3}}-P(\mathcal{Q})\right)
$$

where $W_{\text {tree }}(\Phi, \mathcal{Q}) \equiv \sum_{t} y_{t}(\Phi) \mathcal{Q}^{t}$ is the Wilsonian superpotential of the perturbative theory re-expressed in terms of the gauge-invariant matter condensates $\mathcal{Q}^{t}$ instead of the hidden matter fields $Q^{I}$ themselves. The $P(\mathcal{Q})$ in eq. (4.32) is a holomorphic function of the hidden matter condensates $\mathcal{Q}^{t}$; it does not depend on the gaugino condensate $\widehat{\mathcal{U}}$ or on the moduli. In the limiting case of a hidden sector without matter fields, $P$ is simply a constant and eq. (4.32) reduces to eq. (4.6).

Notice that both the general form of the superpotential (4.32) and the way it determines $\langle\widehat{\mathcal{U}}\rangle(\Phi),\left\langle\mathcal{Q}^{t}\right\rangle(\Phi)$ and $W_{\bmod }(\Phi)$ (cf. eqs. (4.29) and (4.30)) follow from a single physical demand: Whenever the vacuum state of a hidden sector is stable and supersymmetric, the effective potential for the moduli generated by that hidden sector must be consistent with the local supersymmetry. This general argument even fixes the value of the coefficient $\mathbf{c}$ to be exactly as in eq. (2.21). Consequently, regardless of the exact spectrum of the hidden matter fields, we can always re-write the effective superpotential in a manifestly Kähler-invariant form

$$
\begin{aligned}
\widetilde{W}_{\mathrm{eff}}(\varphi, \Phi, \mathcal{U}, \mathcal{Q}) & \equiv \varphi^{3} W_{\mathrm{eff}}(\Phi, \widehat{\mathcal{U}}, \mathcal{Q}) \\
& =\widetilde{W}_{\text {tree }}(\varphi, \Phi, \mathcal{Q})+\frac{\mathcal{U}}{4} \tilde{f}_{W}(\varphi, \Phi)-\frac{\mathcal{U}}{32 \pi^{2}}\left(\mathbf{c} \log \frac{\mathcal{U}}{\Lambda^{3}}-P(\mathcal{Q})\right)
\end{aligned}
$$

$\left(\widetilde{W}_{\text {tree }} \equiv \varphi^{3} W_{\text {tree }}\right)$

The function $P(\mathcal{Q})$ is not determined by the above arguments; nevertheless, knowing that the non-perturbative part of the effective superpotential is completely blind to the couplings of the theory helps us to turn symmetry considerations into severe constraints upon the form of that function. ${ }^{[16-19,48]}$ Indeed, eq. (4.33) must be invariant under all exact symmetries of the EQFT (for spontaneously broken 
symmetries, the superpotential is invariant but the solutions of eqs. (4.29) are not); in particular, the function $P(\mathcal{Q})$ must be invariant. However, because $P(\mathcal{Q})$ is blind to the non-gauge couplings of the theory, it would remain invariant even if we were to change those couplings in an asymmetric way. Therefore, $P$ must respect any 'flavor' symmetry of the strong gauge interactions, no matter how badly this symmetry may be broken by the other interactions.

The R-symmetries and even the anomalous flavor symmetries of the gauge interactions also impose constraints on the $P(\mathcal{Q})$. For such symmetries we may change the continually-adjustable couplings of the hidden sector until the flavor symmetry becomes an exact Kähler-symmetry and the Adler-Bell-Jackiw-Konishi anomaly of the gauge interactions is exactly cancelled by the Wilsonian gauge coupling transforming as in eq. (3.19). After such deformation, we must have an invariant $\widetilde{W}_{\text {eff }}$; according to eq. (4.33), this requires the invariance of the combination $8 \pi^{2} \tilde{f}_{W}(\varphi, \Phi)+P(\mathcal{Q})$. (We are using eq. (4.33) rather than (4.32) because the $\widetilde{W}_{\text {eff }}$ and the $\mathcal{U}$ are Kähler-invariant while the $W_{\text {eff }}$ and the $\widehat{\mathcal{U}}$ are not.) The transformation rule for the $\tilde{f}_{W}$ is just eq. (3.19) without the Kähler term; hence, $P(\mathcal{Q})$ should transform according to

$$
P\left(\mathcal{Q}^{\prime}\right)=P(\mathcal{Q})+\sum_{r}^{\substack{\text { hidden } \\ \text { matter }}} 2 T(r) \log \operatorname{det} \Upsilon^{(r)}
$$

Again, because of the coupling-blindness of the $P(\mathcal{Q})$, eq. (4.34) must hold regardless of how badly the flavor symmetry in question may be broken by the non-gauge interactions.

In many cases, eq. (4.34) completely determines the form of $P(\mathcal{Q})$ (except for an additive constant). For example, consider a SQCD-like hidden sector with $N_{C}$ colors and $N_{F}$ flavors. For $N_{F}<N_{C}$, the only independent relevant chiral

\footnotetext{
* This is precisely what happens to the symmetries arising from the target-space modular invariance in string theory. ${ }^{[24,28]}$
} 
condensates of the theory are $\mathcal{Q}^{I J}=Q_{L}^{I} \cdot Q_{R}^{J}$. (This is not true for theories with $N_{F} \geq N_{C}$, which are discussed later in this section.) Under the $S U\left(N_{F}\right) \times$ $S U\left(N_{F}\right)$ flavor symmetry of the theory, the $\mathcal{Q}^{I J}$ form a single irreducible $\left(N_{F}, N_{F}\right)$ representation; therefore, all $S U\left(N_{F}\right) \times S U\left(N_{F}\right)$ invariant holomorphic functions of these condensates depend only on the determinant of the $\mathcal{Q}^{I J}$ matrix. For the vector $U(1)$ symmetry and for the pure-R symmetry eq. (4.34) is trivially satisfied, but the constraint imposed by eq. (4.34) for the anomalous axial symmetry has a unique solution, namely $P\left(\operatorname{det}\left\|\mathcal{Q}^{I J}\right\|\right)=\log \operatorname{det}\left\|\mathcal{Q}^{I J}\right\|+$ const. Therefore,

$$
\begin{aligned}
W_{\text {eff }}= & W_{\text {tree }}(\Phi, \mathcal{Q})+\frac{\widehat{\mathcal{U}}}{4} f^{W}(\Phi) \\
& +\frac{\widehat{\mathcal{U}}}{32 \pi^{2}}\left(\left(N_{C}-N_{F}\right) \log \frac{\widehat{\mathcal{U}}}{\Lambda^{3}}+\log \frac{\operatorname{det}\left\|\mathcal{Q}^{I J}\right\|}{\Lambda^{2 N_{F}}}+C\right)
\end{aligned}
$$

where $C$ is a constant, presumably of order $O(1)$. From the low energy point of view, the Wilsonian gauge coupling $f^{W}$, the ultraviolet cutoff $\Lambda$ and the unknown constant $C$ can all be combined into a single parameter, which can be identified with the confinement scale of the theory. In terms of that parameter, eq. (4.35) becomes identical to the well-known Taylor-Veneziano-Yankielowicz superpotential for SQCD. ${ }^{[16]}$

In the arguments leading to eq. (4.35), the assumption was that the confinement scale $\mu$ of the hidden sector is much smaller than $\Lambda$ or $M_{\mathrm{Pl}}$, or, rather, that all the condensates are much smaller than $\Lambda$ or $M_{\mathrm{Pl}}$ to the appropriate power. However, there is no need to assume $\langle\widehat{\mathcal{U}}\rangle=O\left(\mu^{3}\right)$ or $\left\langle\mathcal{Q}^{I J}\right\rangle=O\left(\mu^{2}\right)$, and indeed, the superpotential (4.35) suffers no corrections when some of the condensates are much larger or much smaller than the others. This observation can be used to establish a relationship between the constants $C$ of hidden sectors with different numbers

$\dagger$ Because the Kähler factor cancels out of eq. (4.34), this equation is automatically satisfied by the pure-R symmetry of the hidden sector, regardless of whether this symmetry is anomalous or not. Hence, for any supersymmetric hidden sector, one should only check eq. (4.34) for the flavor symmetries that commute with SUSY. 
of colors and flavors. ${ }^{[18,19]}$ Suppose a 'squark' $Q_{L}^{1}$ and an 'antisquark' $Q_{R}^{1}$ somehow acquire Higgs-like VEVs that are much larger than the confinement scale $\mu$ (this can be easily arranged by a judicious choice of the $\left.W_{\text {tree }}\right)$. The superpotential (4.35) does not care that the theory has a threshold, all it sees is that $\left\langle\mathcal{Q}^{11}\right\rangle \approx$ $\left\langle Q_{L}^{1}\right\rangle\left\langle Q_{R}^{1}\right\rangle \gg \mu^{2}$. On the other hand, the physics of sub-threshold energies can also be described in terms of a low-energy effective theory, which for the problem at hand has $N_{C}^{\prime}=N_{C}-1, N_{F}^{\prime}=N_{F}-1$ and a Wilsonian gauge coupling $f_{W}^{\prime}=$ $f^{W}+\left(1 / 8 \pi^{2}\right) \log \left(\left\langle Q_{L}^{1}\right\rangle\left\langle Q_{R}^{1}\right\rangle / 2 \Lambda^{2}\right)$ (cf. eq. (3.28)). It is easy to see that both points of view lead to exactly the same effective superpotential for the $\widehat{\mathcal{U}}$ and the $\mathcal{Q}^{I J}(I, J \geq 2)$, provided $C\left(N_{C}-1, N_{F}-1\right)=C\left(N_{C}, N_{F}\right)+\log 2$. Now suppose that one of the quark flavors, e.g., $Q^{1}$ is very heavy. Again, there are two ways to analyse this case: Either first integrate out the heavy flavor from the perturbative low-energy EQFT and then use eq. (4.35) for that theory, or first write down eq. (4.35) for the theory with all $N_{F}$ flavors and then integrate out all the condensates involving the heavy fields. Fairly straightforward algebra shows that again the perturbative eq. (3.28) and the non-perturbative eq. (4.35) are entirely consistent with each other, provided $C\left(N_{C}, N_{F}-1\right)=C\left(N_{C}, N_{F}\right)-1-\log \left(-32 \pi^{2}\right)$, and hence

$$
\begin{aligned}
W_{\text {eff }}= & W_{\text {tree }}(\Phi, \mathcal{Q})+\frac{1}{4} \widehat{\mathcal{U}} f^{W}(\Phi) \\
& +\frac{\widehat{\mathcal{U}}}{32 \pi^{2}}\left(\left(N_{C}-N_{F}\right) \log \frac{\widehat{\mathcal{U}}}{-64 \pi^{2} e \Lambda^{3}}+\log \frac{\operatorname{det}\left\|\mathcal{Q}^{I J}\right\|}{\left(2 \Lambda^{2}\right)^{N_{F}}}+C_{0}\right),
\end{aligned}
$$

where $C_{0}$ is a universal constant, the same for all $N_{F}<N_{C}$. The same constant $C_{0}$ also appears in the effective superpotentials of other hidden sectors, for example when the hidden gauge group $G=S O\left(N_{C}\right)$ rather than $S U\left(N_{C}\right) \cdot{ }^{[18]}$ For the hidden matter that forms $N_{F}$ vector representations of the $S O\left(N_{C}\right)$ we can repeat 
our previous argument almost verbatim. The result is:

$$
\begin{aligned}
W_{\text {eff }}= & W_{\text {tree }}(\Phi, \mathcal{Q})+\frac{1}{4} \widehat{\mathcal{U}} f^{W}(\Phi) \\
& +\frac{\widehat{\mathcal{U}}}{32 \pi^{2}}\left(\left(N_{C}-2-N_{F}\right) \log \frac{\widehat{\mathcal{U}}}{-64 \pi^{2} e \Lambda^{3}}+\log \frac{\operatorname{det}\left\|\mathcal{Q}^{I J}\right\|}{\left(2 \Lambda^{2}\right)^{N_{F}}}+C_{0}\right)
\end{aligned}
$$

where $\mathcal{Q}^{I J}$ is now a symmetric matrix originating in $Q^{I} \cdot Q^{J}$ and $C_{0}$ is exactly the same constant as in eq. (4.36) because for the case of $S O(6)$ with $N_{F}=0$ the hidden sector is identical to the $S U(4)$ SSYM theory (also with $N_{F}=0$ ). The actual computation of $C_{0}$ requires an honest calculation of a non-perturbative VEV in a strongly interacting theory which is outside the scope of this paper. What we find remarkable is that one such calculation would be sufficient to evaluate all the relevant non-perturbative VEVs in a whole class of strongly interacting theories! ${ }^{[18]}$

The methods just presented (relating different supersymmetric gauge theories) can also be used to completely specify the effective superpotential for $S U\left(N_{C}\right)$ gauge theories with $N_{F} \geq N_{C}$. The well known difficulty with such theories is that they have more independent relevant matter condensates than just the $\mathcal{Q}^{I J} \equiv Q_{L}^{I} \cdot Q_{R}^{J} ;^{[25,19]}$ for example, in the $N_{F}=N_{C}$ case there are two additional condensates, $\mathcal{Q}^{L} \equiv \operatorname{det} Q_{L}$ and $\mathcal{Q}^{R} \equiv \operatorname{det} Q_{R}{ }^{\star}$. Although the operators $\mathcal{Q}^{L}, \mathcal{Q}^{R}$ and $\mathcal{Q}^{I J}$ are related to each other via $\mathcal{Q}^{L} \mathcal{Q}^{R}=\operatorname{det}\left\|\mathcal{Q}^{I J}\right\|$, the non-perturbative effects in the quantum theory lead to $\left\langle\mathcal{Q}^{L}\right\rangle\left\langle\mathcal{Q}^{R}\right\rangle \neq \operatorname{det}\left\|\left\langle\mathcal{Q}^{I J}\right\rangle\right\|$. This is why in the effective superpotential (4.32), the classical variables $\mathcal{Q}^{L}, \mathcal{Q}^{R}$ and $\mathcal{Q}^{I J}$ should be regarded as independent. Moreover, the condensates $\mathcal{Q}^{L}$ and $\mathcal{Q}^{R}$ are always relevant (even for $N>3$ when the couplings associated with the operators $\mathcal{Q}^{L}$ and $\mathcal{Q}^{R}$ are non-renormalizable). This follows from the observation that the theory may have SUSY-preserving Higgs-like VEVs $\left\langle Q_{L, s}^{I}\right\rangle \propto \delta_{s}^{I},\left\langle Q_{R}\right\rangle=0$ or vice verse, and the simplest holomorphic, gauge-invariant order parameters for this behavior of the theory are the $\mathcal{Q}^{L}$ and the $\mathcal{Q}^{R}$.

$\star$ In fully indexed notations, $\mathcal{Q}^{L} \equiv \epsilon^{s_{1}, \ldots, s_{N}} Q_{s_{1}}^{1} \cdots Q_{s_{N}}^{N}$ where $N=N_{F}=N_{C}, s_{1}, \ldots, s_{N}$ are the gauge indices, and ditto for the $\mathcal{Q}^{R}$. 
Our first step towards constructing the $P\left(\mathcal{Q}^{I J}, \mathcal{Q}^{L}, \mathcal{Q}^{R}\right)$ function for a hidden sector with $N_{F}=N_{C}=N$ is to use eqs. (4.34) for the $S U(N)_{L} \times S U(N)_{R} \times U(1)_{V} \times$ $U(1)_{A}$ flavor symmetry, under which the condensates $\mathcal{Q}^{I J}, \mathcal{Q}^{L}$ and $\mathcal{Q}^{R}$ transform as $(\mathbf{N}, \overline{\mathbf{N}}, 0,+2),(\mathbf{1}, \mathbf{1},+1,+N)$ and $(\mathbf{1}, \mathbf{1},-1,+N)$. Under these circumstances, eq. (4.34) yields

$$
P\left(\mathcal{Q}^{I J}, \mathcal{Q}^{L}, \mathcal{Q}^{R}\right)=\log \frac{h\left(\operatorname{det}\left\|\mathcal{Q}^{I J}\right\|, \mathcal{Q}^{L} \mathcal{Q}^{R}\right)}{\left(2 \Lambda^{2}\right)^{N}}
$$

where $h$ is a homogenous function of degree one, but its specific form cannot be determined from the symmetry arguments alone. ${ }^{[19]}$ (For obvious reasons, the product $\mathcal{Q}^{L} \mathcal{Q}^{R}$ has exactly the same quantum numbers as the $\operatorname{determinant} \operatorname{det}\left\|\mathcal{Q}^{I J}\right\|$.) Instead, let us compare the $h$ functions for theories with different values of $N$. As before, we consider the limit of large Higgs-like VEVs $\left\langle Q_{L}^{1}\right\rangle$ and $\left\langle Q_{R}^{1}\right\rangle$, in which $\mathcal{Q}^{L}=\left\langle Q_{L}^{1}\right\rangle \mathcal{Q}^{\prime L}, \mathcal{Q}^{R}=\left\langle Q_{R}^{1}\right\rangle \mathcal{Q}^{\prime R}$ and $\operatorname{det}\left\|\mathcal{Q}^{I J}\right\|=\left\langle Q_{L}^{1}\right\rangle\left\langle Q_{R}^{1}\right\rangle \operatorname{det}^{\prime}\left\|\mathcal{Q}^{I J}\right\|$, where the primes refer to the condensates of the sub-threshold theory with $N_{F}^{\prime}=N_{C}^{\prime}=$ $N-1$. Substituting these values into eq. (4.32) and using the homogeneity of $h$ in eq. (4.38), we find that the sub-threshold theory has

$W_{\text {eff }}^{\prime}-W_{\text {tree }}^{\prime}=\frac{1}{4} f^{W} \widehat{\mathcal{U}}+\frac{\widehat{\mathcal{U}}}{32 \pi^{2}} \log \frac{\left.\left\langle Q_{L}^{1}\right\rangle\left\langle Q_{R}^{1}\right\rangle\right)}{2 \Lambda^{2}}+\frac{\widehat{\mathcal{U}}}{32 \pi^{2}} \log \frac{h\left(\operatorname{det}^{\prime}\left\|\mathcal{Q}^{I J}\right\|, \mathcal{Q}^{\prime L} \mathcal{Q}^{\prime R}\right)}{\left(2 \Lambda^{2}\right)^{N-1}}$

(there is no $\widehat{\mathcal{U}} \log \widehat{\mathcal{U}}$ term because $\mathbf{c}=N_{C}^{\prime}-N_{F}^{\prime}=N_{C}-N_{F}=0$ ). The first two terms on the right hand side of this formula can be combined into $\frac{1}{4} f^{\prime W} \widehat{\mathcal{U}}$ and according to eq. (3.28), the $f^{\prime W}$ is precisely the Wilsonian gauge coupling of the sub-threshold effective theory. Similarly, the remaining $\log \left(h /\left(2 \Lambda^{2}\right)^{N-1}\right)$ term can be identified with the non-perturbative superpotential $\log \left(h^{\prime} /\left(2 \Lambda^{2}\right)^{N^{\prime}}\right)$ of the subthreshold theory, which means that both $h$ functions (above and below the threshold) have exactly the same analytic form. An immediate corollary is that the $h$ functions has the same analytic form for all $N_{F}=N_{C}=2,3,4, \ldots$.

Now consider the special case $N_{F}=N_{C}=2$. For this theory $\mathcal{Q}^{L}$ is a squark bilinear, $\mathcal{Q}^{R}$ is an antisquark bilinear, and because $S U(2)$ does not distinguish 
between squarks and antisquarks, it also does not distinguish between these two condensates and the squark-antisquark bilinears $\mathcal{Q}^{I J}$. In fact, the full flavor symmetry of this gauge theory is $S U(4) \times U(1)$ rather than $S U(2) \times S U(2) \times U(1) \times U(1)$ and the bilinear condensates $\mathcal{Q}^{L}, \mathcal{Q}^{R}$ and $\mathcal{Q}^{I J}$ form an irreducible six-dimensional representation of the $S U(4)$. This representation has only one independent invariant, which in our notations looks like $\mathcal{Q}^{11} \mathcal{Q}^{22}-\mathcal{Q}^{12} \mathcal{Q}^{21}-\mathcal{Q}^{L} \mathcal{Q}^{R}$. Comparing this expression to eq. (4.38), we immediately conclude that for $N_{F}=N_{C}=2$,

$$
h \propto \operatorname{det}\left\|\mathcal{Q}^{I J}\right\|-\mathcal{Q}^{L} \mathcal{Q}^{R}
$$

However, we have just argued that the $h$ functions are universal for all theories with $N_{F}=N_{C}$; hence, for all such theories

$$
W_{\text {eff }}=W_{\text {tree }}(\Phi, \mathcal{Q})+\frac{1}{4} \widehat{\mathcal{U}} f^{W}(\Phi)+\frac{\widehat{\mathcal{U}}}{32 \pi^{2}}\left(\log \frac{\operatorname{det}\left\|\mathcal{Q}^{I J}\right\|-\mathcal{Q}^{L} \mathcal{Q}^{R}}{\left(2 \Lambda^{2}\right)^{N}}+C_{0}\right)
$$

(The constant $C_{0}$ in this formula is exactly as in eqs. (4.36) and (4.37); this equality follows from the decoupling of massive flavors and can be proven in the same way as (4.36).)

The theories with $N_{F}>N_{C}$ can be analysed in exactly the same manner. For the sake of notational simplicity, we only discuss the case $N_{F}=N_{C}+1$, in which the independent relevant matter condensates are comprised of $\mathcal{Q}^{I J}, \mathcal{Q}_{I}^{L}$ and $\mathcal{Q}_{J}^{R^{\star}}$. The $S U\left(N_{F}\right)_{L} \times S U\left(N_{F}\right)_{R} \times U(1)_{V} \times U(1)_{A}$ flavor symmetry of the theory now implies $P(\mathcal{Q})=\log \left(h\left(\operatorname{det}\left\|\mathcal{Q}^{I J}\right\|, \mathcal{Q}_{I}^{L} \mathcal{Q}^{I J} \mathcal{Q}_{J}^{R}\right) / \Lambda^{2 N_{F}}\right)$, where as before $h$ is a homogeneous function of degree one. Again, the arguments using the decoupling

$\star$ The definition of the $\mathcal{Q}_{I}^{L}$ is $\left(1 / N_{C} !\right) \epsilon^{s_{1}, \ldots, s_{N_{C}}} \epsilon_{I_{1}, \ldots I_{N_{C}, I}} Q_{L, s_{1}}^{I_{1}} \cdots Q_{L, s_{N_{C}}}^{I_{N_{C}}}$; the $\mathcal{Q}_{J}^{R}$ is defined likewise. For $N_{F}>N_{C}+1$ the $\mathcal{Q}^{L}$ and the $\mathcal{Q}^{R}$ are $\left(N_{F}-N_{C}\right)$-index tensors of the respective $S U\left(N_{F}\right)$ flavor groups and the notations become somewhat unwieldy. The reasons why all of the $\mathcal{Q}_{\ldots}^{L}, \mathcal{Q}_{\ldots}^{R}$ and $\mathcal{Q}^{I J}$ should be regarded as independent variables in the effective superpotential are exactly as in the $N_{F}=N_{C}$ case; the same goes for the relevancy of all these condensates. 
of large Higgs-like VEVs tell us that $h$ should have the same analytic form for all $N_{C} \geq 2$ as long as $N_{F}=N_{C}+1$, and the specific form of $h$ is fixed by the special case of $N_{C}=2, N_{F}=3$ where the flavor symmetry is extended to the $S U(6) \times U(1)$. The result is

$$
\begin{aligned}
W_{\text {eff }}= & W_{\text {tree }}(\Phi, \mathcal{Q})+\frac{1}{4} \widehat{\mathcal{U}} f^{W}(\Phi) \\
& +\frac{\widehat{\mathcal{U}}}{32 \pi^{2}}\left(-\log \frac{\widehat{\mathcal{U}}}{-64 \pi^{2} e \Lambda^{3}}+\log \frac{\operatorname{det}\left\|\mathcal{Q}^{I J}\right\|-\mathcal{Q}_{I}^{L} \mathcal{Q}^{I J} \mathcal{Q}_{J}^{R}}{\left(2 \Lambda^{2}\right)^{N_{F}}}+C_{0}\right)
\end{aligned}
$$

Note that the coefficient $\left(N_{C}-N_{F}\right)$ of the $\widehat{\mathcal{U}} \log \widehat{\mathcal{U}}$ term is now negative, and because of this sign, the superpotential (4.41) leads to a stable vacuum even in the absence

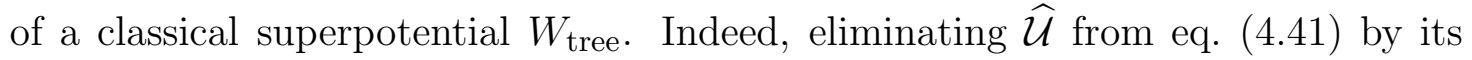
equation of motion leads to an effective superpotential for the matter condensates that looks like

$$
W_{\mathrm{eff}}(\mathcal{Q}) \propto \mathcal{Q}_{I}^{L} \mathcal{Q}^{I J} \mathcal{Q}_{J}^{R}-\operatorname{det}\left\|\mathcal{Q}^{I J}\right\|
$$

and has no run-away directions: All eqs. (4.29) are satisfied by $\langle\mathcal{Q}\rangle=0$. Furthermore, the superpotential (4.42) contains no mass terms. Hence, as conjectured in ref. [25], the confinement in $N_{F}=N_{C}+1$ theories gives rise to exactly massless composite supermultiplets whose quantum numbers match those of the $\mathcal{Q}_{I}^{L}, \mathcal{Q}_{J}^{R}$ and $\mathcal{Q}^{I J}$ condensates, which is precisely the massless spectrum required by the 't Hooft's flavor anomaly matching conditions for the unbroken chiral symmetry $S U\left(N_{F}\right) \times S U\left(N_{F}\right) \times U(1) \times U(1)$.

Let us summarize the results of this section. The superpotential (4.35) was derived before, using a variety of techniques such as the leading-order instantonic calculations or renormalization-group equations. ${ }^{[16-19]}$ Here we obtain exactly the same superpotential from a different approach, namely the consistency of the effective locally supersymmetric theory for the moduli. Our main result is that this superpotential is exact (modulo corrections suppressed by the negative powers of $M_{\mathrm{Pl}}$ ) and so are other superpotentials derived in this manner. It is precisely the 
exactness of these superpotentials that legitimizes the techniques we used to relate different gauge theories to each other and thus to derive the superpotentials (4.40) and (4.41) for the theories with $N_{F} \geq N_{C}$. In addition, we confirmed that integrating out the entire hidden sector - namely, using eq. (4.30) and inserting the result into eq. (4.10), - indeed gives the correct effective potential for the moduli.

\subsection{Hidden Sectors in String Context.}

In the context of string unification, the main result of the previous section is that for a generic supersymmetric hidden sector, the general form and all the parameters of the effective superpotential (4.33) are exactly calculable in analytic form. The calculation is done in terms of the low-energy EQFT and needs very little input from the string theory, namely the spectrum of the light particles, the relevant superpotential couplings $y_{t}(\Phi)$, and the Wilsonian gauge coupling $f^{W}(\Phi)$. The spectrum and the superpotential are determined at the tree level of the heterotic string whereas $f^{W}$ requires a one-string-loop calculation (plus some treelevel data, to separate the $f^{W}$ from the non-holomorphic terms in $\left.\left\{g^{-2}\right\}^{1-\text { loop }}\right)^{[9,10,28]}$ Consequently, without ever performing higher loop calculations, we can obtain an analytic form of the non-perturbative effective superpotential $W_{\text {mod }}$ for the moduli fields and be sure that it is exact.

In this section, we present several examples of analytically computing $W_{\bmod }$ in terms of the holomorphic Wilsonian couplings of the theory. In our examples, the hidden matter fields are exactly massless (perturbatively), but the chiral symmetry is broken by the Yukawa couplings or by the non-renormalizable terms in $W_{\text {tree}}$. Such behavior is quite rare in ordinary GUTs and thus did not receive much attention in the literature. In string unification, however, masslessness without a chiral symmetry is the norm, and we believe this phenomenon deserves a closer look from the effective superpotential point of view. ${ }^{[24]}$

Our first example is a $S U(3)$ gauge theory with three flavors of triplets $Q_{L}^{I}$ and anti-triplets $Q_{R}^{J}$ and with 'baryon' number violating Yukawa self-couplings of the 
matter fields,

$$
W_{\text {tree }}=Y_{L}(\Phi) \operatorname{det}\left\|Q_{L}\right\|+Y_{R}(\Phi) \operatorname{det}\left\|Q_{R}\right\|
$$

Substituting (4.43) into eq. (4.40) for $N_{F}=N_{C}$ and solving the resulting eqs. (4.29) and (4.30) result in

$$
\begin{gathered}
W_{\bmod }(\Phi)=2 \Lambda^{3}\left[-8 e^{-C_{0}} e^{-8 \pi^{2} f^{W}(\Phi)} Y_{L}(\Phi) Y_{R}(\Phi)\right]^{1 / 2}, \\
\langle\widehat{\mathcal{U}}\rangle=-32 \pi^{2} \Lambda^{3}\left[-8 e^{-C_{0}} e^{-8 \pi^{2} f^{W}(\Phi)} Y_{L}(\Phi) Y_{R}(\Phi)\right]^{1 / 2}, \\
\left\langle\mathcal{Q}^{L}\right\rangle \equiv\left\langle\operatorname{det}\left\|Q_{L}\right\|\right\rangle=\Lambda^{3}\left[-8 e^{-C_{0}} e^{-8 \pi^{2} f^{W}(\Phi)} Y_{L}^{-1}(\Phi) Y_{R}(\Phi)\right]^{1 / 2}, \\
\left\langle\mathcal{Q}^{R}\right\rangle \equiv\left\langle\operatorname{det}\left\|Q_{R}\right\|\right\rangle=\Lambda^{3}\left[-8 e^{-C_{0}} e^{-8 \pi^{2} f^{W}(\Phi)} Y_{L}(\Phi) Y_{R}^{-1}(\Phi)\right]^{1 / 2}, \\
\operatorname{det}\left\|\left\langle\mathcal{Q}^{I J}\right\rangle\right\| \equiv \operatorname{det}\left\|\left\langle Q_{L}^{I} \cdot Q_{R}^{J}\right\rangle\right\|=0 .
\end{gathered}
$$

The individual VEVs $\left\langle\mathcal{Q}^{I J}\right\rangle$ are undetermined, but adding quartic or other nonrenormalizable couplings to the tree-level superpotential (4.43) would force them to vanish without affecting any of the results (4.44). Without such non-renormalizable couplings, the theory has an accidental $S U(3) \times S U(3)$ chiral symmetry, which remains unbroken by the strong interactions; instead, the theory forms a massless composite matter multiplet with the quantum numbers of $\mathcal{Q}^{I J}$, in full agreement with the 't Hooft's flavor anomaly matching conditions. On the other hand, the abelian flavor symmetries of the gauge theory are explicitly broken by the Yukawa couplings $Y_{L}$ and $Y_{R}$, which are necessary for the stability of the condensate VEVs (4.44): In the limit $Y_{L} \rightarrow 0, Y_{R}$ fixed, we have $\left\langle\mathcal{Q}^{L}\right\rangle \rightarrow \infty$ and for $Y_{R} \rightarrow 0, Y_{L}$ fixed, $\left\langle\mathcal{Q}^{R}\right\rangle$ diverges; in the double limit $Y_{L}, Y_{R} \rightarrow 0$, both $\left\langle\mathcal{Q}^{L}\right\rangle$ and $\left\langle\mathcal{Q}^{R}\right\rangle$ become undetermined.

\footnotetext{
$\star$ This type of a hidden sector often shows up in string models whose construction involves modding out by discrete $Z_{3}$ symmetries.
} 
Unless the Yukawa couplings $Y_{L}$ and $Y_{R}$ are unusually small, all the nonvanishing condensates (4.44) are comparable in magnitude and can be used as a crude estimate of the confinement scale of the theory:

$$
\left\langle\mathcal{Q}^{L}\right\rangle \simeq\left\langle\mathcal{Q}^{R}\right\rangle \simeq\langle\widehat{\mathcal{U}}\rangle \simeq\left[\Lambda e^{-8 \pi^{2} f^{W} / 6}\right]^{3} \sim \mu^{3}
$$

where the factor $1 / 6$ in the exponential is related to $\mathbf{b}=-6$ for a theory with $N_{C}=N_{F}=3$. A more accurate formula for the physically normalized confinement scale would be

$$
\mu \simeq\left[\Lambda e^{-8 \pi^{2} f^{W} / 6}\right] e^{\kappa^{2} K / 6}
$$

where the Kähler factor appears exactly as in eq. (4.3) and for exactly the same physical reason. Unfortunately, the numerical coefficient missing from eq. (4.46) is not as easy to calculate as that for the matter-less SSYM theories.

The fact that all condensates (in eq. (4.45)) are proportional to a single scale is characteristic of hidden sectors in which all the important superpotential couplings are renormalizable. As a more generic example of this behavior, consider an $S U\left(N_{C}\right)$ with $N_{F}<N_{C}$ flavors and some gauge-singlet matter fields with Yukawa coupling to the 'quarks' and 'antiquarks' and also to each other. For notational simplicity, we present the case of $N_{F}=1$ and only one matter singlet $Q_{1}$; thus,

$$
W_{\text {tree }}=Y(\Phi) Q_{1} Q_{L} Q_{R}-\frac{1}{3} Y_{1}(\Phi) Q_{1}^{3}
$$

Supersymmetric EQFTs with gauge-singlet matter fields are governed by a simple rule: The only independent condensates involving the gauge singlet fields are those 
fields themselves. Furthermore, the non-perturbative part of the effective superpotential cannot involve the gauge singlet fields. Consequently, the non-perturbative superpotential for the theory at hand is exactly the Taylor-Veneziano-Yankielowicz superpotential for $N_{F}=1$. Combining together eqs. (4.47) and (4.36), we find

$$
\begin{gathered}
W_{\text {mod }}(\Phi)=\left(N_{C}-\frac{1}{3}\right) \Lambda^{3}\left[2^{3 N_{C}} e^{-3 C_{0}} e^{-24 \pi^{2} f^{W}(\Phi)} Y^{3}(\Phi) Y_{1}^{-1}(\Phi)\right]^{1 /\left(3 N_{C}-1\right)}, \\
\langle\widehat{\mathcal{U}}\rangle=-32 \pi^{2} \Lambda^{3}\left[2^{3 N_{C}} e^{-3 C_{0}} e^{-24 \pi^{2} f^{W}(\Phi)} Y^{3}(\Phi) Y_{1}^{-1}(\Phi)\right]^{1 /\left(3 N_{C}-1\right)} \\
\langle\mathcal{Q}\rangle \equiv\left\langle Q_{L} \cdot Q_{R}\right\rangle=\Lambda^{2}\left[2^{2 N_{C}} e^{-2 C_{0}} e^{-16 \pi^{2} f^{W}(\Phi)} Y^{3-3 N_{C}}(\Phi) Y_{1}^{N_{C}-1}(\Phi)\right]^{1 /\left(3 N_{C}-1\right)} \\
\text { and }\left\langle Q_{1}\right\rangle=\Lambda\left[2^{N_{C}} e^{-C_{0}} e^{-8 \pi^{2} f^{W}(\Phi)} Y(\Phi) Y_{1}^{-N_{C}}(\Phi)\right]^{1 /\left(3 N_{C}-1\right)}
\end{gathered}
$$

Again, for $O(1)$ Yukawa couplings $Y$ and $Y_{1}$ we find crude similarity between the magnitudes of the condensates and the appropriate powers of the confinement scale:

$$
\langle\widehat{\mathcal{U}}\rangle^{1 / 3} \simeq\langle\mathcal{Q}\rangle^{1 / 2} \simeq\left\langle Q_{1}\right\rangle \simeq \Lambda e^{-8 \pi^{2} f^{W} /\left(3 N_{C}-1\right)} \sim \mu
$$

(for the theory at hand, $\mathbf{b}=3 N_{C}-1$; a more accurate formula for the confinement scale $\mu$ involves the Kähler factor, as in eq. (4.45)). Notice that both of the Yukawa

* The physical reason for this rule is the fact that all of the physical couplings of the quantum degrees of freedom contained in the singlet fields can be rendered arbitrarily weak by adjusting the non-holomorphic parameters of the theory, namely the $Z^{(1)}$ matrix for the singlets. In the $Z^{(1)} \rightarrow \infty$ limit, the quantum nature of the singlets becomes irrelevant and only the Higgs-like VEVs remain to complicate the theory, but these VEVs obey the classical relations such as

$$
\left\langle Q_{1}^{3}\right\rangle=\left\langle Q_{1}\right\rangle^{3}, \quad\left\langle Q_{1} Q_{L} Q_{R}\right\rangle=\left\langle Q_{1}\right\rangle\left\langle Q_{L} Q_{R}\right\rangle,
$$

etc.. In a supersymmetric vacuum, expectation values of chiral operators cannot depend on the non-holomorphic couplings. Therefore, equations such as (4.48) must always hold true, even when the $Z^{(1)}$ factors are small and the physical Yukawa couplings of the theory are strong.

$\dagger$ This follows from the same argument: In the $Z^{(1)} \rightarrow \infty$ limit the singlets act like moduli rather than interacting matter fields and hence should only enter the effective superpotential (4.32) through its classical part $W_{\text {tree. }}$ Since the entire effective superpotential is blind to the $Z$ factors, $P(\mathcal{Q})$ remains singlet-independent even when the physical couplings of the singlets are strong. 
couplings $Y$ and $Y_{1}$ are necessary for the stability of the condensates (4.49): in the $Y \rightarrow \infty$ limit, the squark and the antisquark develop large Higgs-like expectation values, while for $Y_{1} \rightarrow \infty$ it is the singlet $\operatorname{VEV}\left\langle Q_{1}\right\rangle$ that grows out of control.

In our final example we dispense with both the singlet fields and the Yukawa couplings; instead, the Higgs-like VEVs of the massless squarks and antisquarks are controlled by the non-renormalizable couplings. The simplest theory of this kind is $S U\left(N_{C}\right)$ with $N_{F}=1$ and a tree-level superpotential

$$
W_{\text {tree }}=\frac{\gamma(\Phi)}{2 \Lambda}\left(Q_{L} \cdot Q_{R}\right)^{2}
$$

in the context of string unification, $\Lambda$ is usually the same as $M_{\mathrm{Pl}}$. The nonrenormalizable couplings are relevant only when the corresponding operators have unexpectedly large VEVs; for the problem at hand, the quartic coupling (4.51) is relevant if $\left\langle\left(Q_{L} \cdot Q_{R}\right)^{2}\right\rangle=O\left(\Lambda \mu^{3}\right) \gg \mu^{4}$. Such large VEVs are characteristic of the Higgs limit of the theory, $\langle Q\rangle \gg \mu$. However, because of the classical nature of the Higgs mechanism, it does not contribute to expressions such as $\left\langle\left(Q_{L} \cdot Q_{R}\right)^{2}\right\rangle-\left\langle\left(Q_{L} \cdot Q_{R}\right)\right\rangle^{2}$. Consequently, the difference between the square of the squark-antisquark bilinear condensate and the quartic condensate is irrelevant, and in the effective superpotential (4.28) we do not need an independent variable for the quartic condensate. Thus $W_{\text {eff }}$ is given by eq. (4.36), with $N_{F}=1$ and $W_{\text {tree }}=(\gamma / 2 \Lambda) \mathcal{Q}^{2}$, where $\mathcal{Q} \equiv\left(Q_{L} \cdot Q_{R}\right)$ is the usual bilinear condensate. After going through the usual algebra we arrive at

$$
\begin{gathered}
W_{\bmod }(\Phi)=\left(N-\frac{1}{2}\right) \Lambda^{3}\left[2^{2 N_{C}} e^{-2 C_{0}} e^{-16 \pi^{2} f^{W}(\Phi)} \gamma(\Phi)\right]^{1 /(2 N-1)} \\
\langle\widehat{\mathcal{U}}\rangle=-32 \pi^{2} \Lambda^{3}\left[2^{2 N_{C}} e^{-2 C_{0}} e^{-16 \pi^{2} f^{W}(\Phi)} \gamma(\Phi)\right]^{1 /(2 N-1)} \\
\langle\mathcal{Q}\rangle \equiv\left\langle Q_{L} \cdot Q_{R}\right\rangle=\Lambda^{2}\left[2^{N_{C}} e^{-C_{0}} e^{-8 \pi^{2} f^{W}(\Phi)} \gamma^{1-N}(\Phi)\right]^{1 /(2 N-1)}
\end{gathered}
$$

Note that the Higgs-like VEVs of the squark and the antisquark are indeed very 
large compared to the confinement scale of the theory. To a crude approximation,

$$
\langle\widehat{\mathcal{U}}\rangle^{1 / 3} \sim \mu, \quad \text { but } \quad\left\langle Q_{L}\right\rangle,\left\langle Q_{R}\right\rangle \sim \sqrt[4]{\mu^{3} \Lambda}
$$

as usual, this formula assumes $\gamma \sim O(1)$ and ignores the Kähler factor in the confinement scale $\mu$. Below the Higgs threshold we effectively have a matter-less $S U\left(N_{C}-1\right)$ SSYM theory, so we can use eq. (4.3) to write down an exact formula

for the confinement scale of the model at hand; all we need is eq. (3.28) for the threshold effect and eq. (4.52) for the un-normalized Higgs VEVs. Combining all the factors together, we have

$$
\mu=\left[\frac{8 \pi^{2} e^{1-C_{0} /\left(N_{C}-1\right)\left(2 N_{C}-1\right)}}{N_{C}-1}\right]^{1 / 3} \gamma^{1 /\left(6 N_{C}-3\right)} \Lambda \exp \left(-\frac{8 \pi^{2} \operatorname{Re} f^{W}}{3 N_{C}-\frac{3}{2}}+\frac{1}{6} \kappa^{2} K\right)
$$

The denominator of the $\operatorname{Re} f^{W}$ term in the exponential reflects the fact that the theory has a threshold between $\mu$ and $\Lambda$ : $3 N_{C}-\frac{3}{2}$ is the appropriately weighted (cf. eq. (4.53)) average of the $\beta$-function coefficient $\mathbf{b}^{\prime}=3\left(N_{C}-1\right)$ for the effective sub-threshold theory and of $\mathbf{b}=3 N_{C}-1$ for the unbroken theory above the threshold.

\subsection{Families of EQFTs, Vacuum Instabilities and SUSY Breaking.}

In the previous sections, we discussed how the hidden sectors of the unified theory generate a non-perturbative effective potential for the moduli fields. In this section, we discuss the physical implications of this potential but to put the discussion into a wide enough context, we begin by considering the domains of validity of the low-energy EQFTs and what happens when we try to push an EQFT outside its proper domain.

Consider a generic unified theory that has several alternative vacua. Generally, expanding around each individual vacuum yields a different spectrum of light 
particles and hence a different low-energy EQFT. The situation becomes more complicated when the vacua of the unified theory form continuous families. In this case, the domain of validity of the EQFT obtained by expanding around any particular vacuum also includes the immediate neighborhood of that vacuum and the moduli VEVs $\left\langle\Phi^{i}\right\rangle,\left\langle\bar{\Phi}^{\imath}\right\rangle$ serve as coordinates in this neighborhood. However, when we go far away from the original vacuum, the low energy physics may suffer more changes that could be handled by a mere change of coordinates in the moduli space; instead, re-expansion around the new vacuum may yield a different spectrum of light particles and a different EQFT to describe their low-energy behavior. The key to this discontinuity is that although the particles' masses vary continuously throughout the vacuum space, at some point we have to make a decision whether a particular field is light enough to include in the low-energy EQFT or is heavy enough to integrate out. This decision is necessarily arbitrary to some extent, but not completely so; therefore, a continuous vacuum family of the heterotic string (or other unified theory) may need several distinct EQFTs to describe its low-energy behavior, and the domains of validity of those EQFTs overlap at each other's edges, but cannot be amalgamated into a single domain of a single EQFT.

When a low-energy EQFT approaches the limit of its domain of validity, it usually gives some signals of its impending breakdown. When the moduli VEVs enter a neighborhood of the vacuum space where some normally heavy gauge or matter fields become light or even massless, some Wilsonian couplings of the EQFT become singular. For example, when the additional light fields are charged under some gauge group $G_{a}$, the Wilsonian gauge coupling $f_{a}^{W}$ diverges like the logarithm of the low mass. Going in the opposite direction, we see light fields becoming superheavy, and this phenomenon normally has a low-energy EQFT explanation in

* In string theory, and in particular in Kaluza-Klein compactifications of the ten-dimensional heterotic string, an infinite number of heavy charged fields simultaneously become massless in the 'decompactification' limit when the radius of the 'internal' manifold becomes large. In this case, the couplings of the four-dimensional EQFT diverge like powers (rather than the logarithm) of the appropriate modulus. We shall return to this scenario later in this section. 
terms of unexpectedly large VEVs of some matter scalars $Q^{I}$ : The gauge fields of the symmetries broken by these large $\left\langle Q^{I}\right\rangle$ become heavy via the Higgs mechanism while the non-Higgs matter fields can also get superheavy masses via Yukawa couplings to the large VEVs. When the large VEVs reach the Planck scale, the original EQFT breaks down, not because of the heavy masses, but because we can no longer truncate the expansions (2.2), (2.4) and (2.6) of the Wilsonian couplings of the theory into powers of $Q^{I} / M_{\mathrm{Pl}}$.

Clearly, the VEVs of matter scalars can reach the Planck scale only if they grow along an exactly flat direction of the classical scalar potential; such directions are quite common in supersymmetric EQFTs, especially those derived from the heterotic string. ${ }^{[49]}$ When a flat combination of matter fields does acquire a Plancksized VEV, it behaves exactly like a modulus, and once we integrate out all the fields that become superheavy in the $\left\langle Q^{\text {flat }}\right\rangle=O\left(M_{\mathrm{Pl}}\right)$ regime, the $Q^{\text {flat }}$ becomes a modulus since now it is both flat and neutral (all the gauge symmetries that act upon the $Q^{\text {flat }}$ are too badly broken to retain in the low-energy EQFT). On the other hand, whenever $\left\langle Q^{\text {flat }}\right\rangle$ happens to be small compared to $M_{\mathrm{Pl}}$ and the gauge symmetries that act upon the $Q^{\text {flat }}$ take part in the low-energy EQFT, the $Q^{\text {flat }}$ is not a modulus but a combination of charged matter fields $Q^{I}$. Thus, we see that the distinction between the moduli and the flat combinations of the matter fields is as arbitrary as the boundaries between the validity domains of EQFTs describing the same vacuum family of the unified theory: In each EQFT we know which scalar is a modulus and which is matter, but as we cross from the domain of one EQFT into the domain of another, a modulus may become a combination of matter fields and vice versa.

For example, consider a string orbifold such as $Z_{3}$ and the related Calabi-Yau compactification. ${ }^{[50,47]}$ In the orbifold limit, the gauge group is $S U(3) \otimes E_{6} \otimes E_{8}$ and the only neutral scalars are the nine toroidal moduli and the dilaton. However, among the 243 scalar superfields that transform like 81 triplets of the $S U(3)$ and are neutral with respect to the $E_{6} \otimes E_{8}$, there are 27 exact flat directions. To 
the orbifold-based EQFT, these flat directions are not moduli but matter; in fact they are linear combinations of the charged fields that cannot possibly be moduli. However, because those particular combinations of matter scalar happen to be exact flat directions of the potential, their VEVs are indeterminate, and can take any values from zero to $M_{\mathrm{Pl}}$ and beyond. But when the $S U(3)$-triplets acquire Planck-sized VEVs, the $S U(3)$ gauge bosons become so heavy that there is no longer any sense in including them among the light fields (some charged scalars become super-heavy too). Thus, within the same continuous family of string vacua, we have to switch to a different EQFT, presumably corresponding to an orbifold that is 'blown-up' to a smooth Calabi-Yau manifold; this new EQFT has a smaller gauge group — just the $E_{6} \otimes E_{8}$ - and fewer scalars. However, of the scalars that remain, the 27 flat combinations of the scalars that used to be charged under the $S U(3)$ now become moduli, precisely because any particles to whom these scalars give masses are too heavy to appear in the new EQFT.

Note that of the two EQFTs - one for the orbifold, and one for the smooth compactification - neither is a special case of the other. Although the orbifoldbased EQFT has more fields, the manifold-based EQFT has more moduli, and that means that all the couplings of the theory - the Kähler function (2.2), the superpotential (2.4) and the gauge couplings (2.6) - must be written as analytic functions of all the 36 moduli. While formally we can always expand those functions into powers of the twisted moduli, such an expansion is a power series in $\frac{\text { twisted moduli }}{M_{\mathrm{Pl}}}$ which are not small, and thus truncating the expansion is quite illegitimate. In contrast, the couplings of the orbifold-based EQFT need only be analytic in the toroidal moduli, and it is perfectly legitimate to truncate the expansion into powers of all other fields, including the twisted matter scalars that combine into the twisted moduli.

The previous discussion makes it clear what to do when a hidden sector of a low-energy EQFT does not have a stable vacuum, supersymmetric or otherwise. This problem is common in non-perturbative supersymmetric gauge theories with 
matter: When the classical scalar potential for the hidden matter fields has a flat direction and the non-perturbative effective potential happens to decrease along that direction, the theory has no stable vacuum and a run-away expectation value $\left\langle Q^{\text {flat }}\right\rangle \rightarrow \infty$. In the previous section, we saw that when the would-be run-away direction is only flat in the renormalizable approximation but lifted by the nonrenormalizable couplings, the run-away eventually stops and the theory does have a stable vacuum. A characteristic feature of this scenario is an unusually large scalar VEV, which is nevertheless very small compared to the Planck scale: $\langle Q\rangle \sim$ $\mu^{1-\rho} M_{\mathrm{Pl}}^{\rho}(0<\rho<1)$.

On the other hand, when the run-away happens along a truly flat direction of the perturbative EQFT, it does not stop until $\left\langle Q^{\text {flat }}\right\rangle \geq O\left(M_{\mathrm{Pl}}\right)$, at which point the original EQFT is no longer applicable. Instead, we are now in the domain of some other low-energy EQFT and the former run-away matter field is just a modulus. Thus, our analysis must start over again: Compute the spectrum and couplings of the gauge, matter and moduli fields of the new theory, and then use this information to study the stability properties the hidden sectors, if they are still present. If the new theory again gives rise to a run-away vacuum instability, we must enter the domain of a yet another EQFT. To summarize, run-away matter VEVs in a strongly interacting hidden sector tell us that we are expanding around a wrong perturbative vacuum of the unified theory.

A different kind of vacuum instability is associated with the moduli rather than with the hidden matter fields. Indeed, let us assume that all the strongly interacting hidden sectors have perfectly stable supersymmetric vacua as long as the moduli are frozen; however, once the hidden sectors are integrated out, the effective potential (4.10) might not have a stable minimum. Instead, it may continuously decrease in the same direction throughout the moduli space of the EQFT, and in this case, it is the moduli VEVs that run away. A distinct possibility is that the run-away moduli simply evolve away from the validity domain of the original EQFT and towards the domain of another; the true effective potential does have a stable minimum, 
but it lies within the validity domain of the second EQFT and not the first (or perhaps within the domain of a third EQFT, etc.). In this situation, once one uses the low-energy EQFT for the right area of the vacuum family, the existence of the stable non-perturbative vacuum becomes apparent, but when one starts in a wrong domain, the true minimum of the $\mathcal{V}_{\text {mod }}(\Phi, \bar{\Phi})$ may become invisible and all one can tell is that the moduli VEVs would rather lie elsewhere.

Ideally, one should have a global picture of the non-perturbative effective potential for the entire continuous family of perturbative vacua of the unified theory. If this potential has a global minimum, then this minimum corresponds to the true vacuum of the theory. (Unless the unified theory allows tunneling into an entirely different vacuum family.) Alas, the global picture is seldom available, and in its absence the only alternative is to start with some candidate vacuum, construct the corresponding low-energy EQFT and use it to calculate the $\mathcal{V}_{\text {mod }}(\Phi, \bar{\Phi})$. After that, one follows the moduli VEVs as they evolve towards lower values of this effective potential, and whenever they run away from the validity domain of the original EQFT, new low-energy EQFT comes into play and the cycle repeats until one either reaches a minimum of the effective potential or else runs into a genuine instability. Unfortunately, this procedure is only good for finding local rather than global minima of effective potentials and one should always be aware of the possibility that the true non-perturbative vacuum of the theory lies elsewhere.

Sometimes, a run-away direction has no end; instead, the non-perturbative $\mathcal{V}_{\text {mod }}(\Phi, \bar{\Phi})$ continually decreases along some infinitely long trajectory in the moduli space and there is no mechanism in sight that would stop the runaway. In string unification, the dilaton field $\operatorname{Re} S$ often suffers from this instability because large values of the $\operatorname{Re} S$ correspond to the weak coupling regime of the EQFT, which guarantees that for $\operatorname{Re} S \rightarrow+\infty$ the effective potential $\mathcal{V}_{\bmod }(S, \bar{S})$ always approaches zero asymptotically. ${ }^{[51]}$ For many models, this effective potential is decreasing for large enough $\operatorname{Re} S$, and as the result, the VEV of the dilaton never 
stops growing. In this scenario, the theory has a genuine low-energy instability.

Another kind of a vacuum instability occurs when the non-perturbative effective potentials push the moduli VEVs into a domain where the low-energy physics cannot be described by any four-dimensional EQFT. For example, when the radius $R$ of a Calabi-Yau manifold is pushed by the $\mathcal{V}_{\bmod }(R, \ldots)$ to larger and larger values, the vacuum decompactifies from four spacetime dimensions to ten; in a general string-based theory, similar instabilities may correspond to partial decompactifications (from four dimensions to five or six). A generic sign of impending decompactification is an infinite number of massive fields that become light and it is not always clear which degrees of freedom correspond to the new spacetime dimensions; an example of this behavior in a Calabi-Yau context is the conifold limit of the quintic variety in $\mathrm{CP}^{4} .^{[54]}$

Finally, the run-away moduli may drive the theory into a strong-coupling regime that we do not know how to analyze; in string-based theories this happens whenever the VEV of the dilaton is small. To be precise, one has a two-fold problem whenever the running couplings become strong just below $M_{\mathrm{Pl}}$ : First, a perturbatively cut-off EQFT is ill-defined unless we can do perturbation theory near the cutoff scale. Second, the string theory itself is defined perturbatively and we have no idea how a strongly-coupled four-dimensional string theory may look and what are its low-energy implications. The same is a fortiori true for all other presently known candidate theories of the ultimate unification.

Let us now assume that the low-energy theory does have a stable vacuum and turn our attention to the issue of non-perturbative supersymmetry breaking. In a theory with both moduli and hidden sectors, there are two distinct mechanisms for such breakdown. First, the vacuum state of a strongly-interacting hidden sector may have positive energy density and thus spontaneously break SUSY; this is known to happen in some supersymmetric gauge theories with chiral matter, e.g.,

\footnotetext{
$\star$ For suggestions how to cure this problem see refs. [52,53,28].
} 
an $S U(5)$ with a single 10 and a single $\overline{\mathbf{5}}^{[17]}$ In this scenario - usually referred to as the 'dynamical' SUSY breakdown - the effective potential for the moduli is not given by eq. (4.10) but rather by the coupling-dependent vacuum energy of the hidden sector ${ }^{\dagger}$ :

$\mathcal{V}_{\bmod }(\Phi, \bar{\Phi})=$

When a symmetry is spontaneously broken in some sector of an EQFT, integrating that sector out results in a new EQFT in which the original symmetry appears to be broken explicitly; this rule of quantum field theory applies to all symmetries, and SUSY — rigid or local - is no exception. Consequently, the non-perturbative potential (4.55) generally does not have a supersymmetric form.

The detailed analysis of SUSY-breaking hidden sectors is outside the scope of this article; nevertheless, one can generically estimate the magnitudes of various SUSY-breaking effects. If we assume a stable minimum for the potential (4.55) the moduli scalars generally have masses of the order $O\left(\mu^{2} / M_{\mathrm{Pl}}\right)$ ( $\mu$ is the confinement scale of the hidden sector). At the same time, the gravitino mass is also $O\left(\mu^{2} / M_{\mathrm{Pl}}\right)$ and the cosmological constant is $O\left(\mu^{4}\right)$. It is not clear, how seriously one should take this cosmological constant since at present, all unified theories suffer from this problem. However, any mechanism that adjusts the cosmological constant by an $O\left(\mu^{4}\right)$ amount is liable to change the whole effective potential (4.55) by a comparable amount and thus completely re-arrange its shape. Consequently, any predictions based upon the scenario in which SUSY is spontaneously broken in a hidden sector should be taken with grain of salt.

The second mechanism for a dynamically induced spontaneous breakdown of local supersymmetry proceeds in two stages. At stage one, one or more hidden

\footnotetext{
$\dagger$ Strictly speaking, eq. (4.55) gives only the leading term in the expansion of the $\mathcal{V}_{\text {mod }}$ into powers of (confinement scale) $/ M_{\mathrm{Pl}}$, but since it does not vanish, the sub-leading terms are unimportant.
} 
sectors of the low-energy theory produce gaugino condensates (and, possibly, some matter condensates as well), but they do not break SUSY (i.e., their vacua are supersymmetric and stable). Instead, integrating out those hidden sectors yields an effective superpotential $W_{\bmod }(\Phi)$ for the moduli fields according to the rules we discussed in sections 4.1-4. At stage two we thus have a locally supersymmetric effective theory for the moduli, with a perfectly supersymmetric effective potential (4.10). Nevertheless, this effective potential may lead to spontaneous supersymmetry breakdown among the moduli; a signal for this kind of SUSY breakdown would be a $\operatorname{VEV}\left\langle F^{i}\right\rangle$ of an auxiliary component of a modulus superfield $\Phi^{i[20-24]}$

Just as there is no a priori guarantee for the effective potential (4.10) to have a stable minimum, one cannot a priori determine whether such a minimum is supersymmetric or not. The only way to find this out is to calculate the $W_{\text {mod }}(\Phi)$ from the holomorphic couplings of the hidden sectors, evaluate eq. (4.10) and see where it leads. Similarly, it is a matter of model-by-model calculations to find out whether the value of the effective potential at its minimum vanishes or not; this question has a direct bearing on the cosmological constant problem.

Indeed, consider the magnitudes of the SUSY-breaking effects in this scenario. The natural scale of the non-perturbatively induced $W_{\bmod }(\Phi)$ is $O\left(\mu^{3}\right)(\mu$ again being the confinement scale of the hidden sector). Hence, the natural scale of the scalar potential $(4.10)$ is $O\left(\mu^{6} / M_{\mathrm{Pl}}^{2}\right)$, the moduli masses are $O\left(\mu^{3} / M_{\mathrm{Pl}}^{2}\right)$ and if SUSY is spontaneously broken, then $\left\langle F^{i}\right\rangle=O\left(\mu^{3} / M_{\mathrm{Pl}}^{2}\right)$ and the gravitino mass $m_{3 / 2}$ is also $O\left(\mu^{3} / M_{\mathrm{Pl}}^{2}\right)$. Thus, in terms of the gravitino mass, the moduli mass is $O\left(m_{3 / 2}\right)$ and the natural scale of the effective potential is $O\left(m_{3 / 2}^{2} M_{\mathrm{Pl}}^{2}\right)$, exactly like for SUSY broken in a hidden sector. Hence, if the minimal value of the effective potential (4.10) does not at least approximately vanish relative to its natural scale, we have a cosmological constant of the order $O\left(m_{3 / 2}^{2} M_{\mathrm{Pl}}^{2}\right)$ and thus the usual danger that an unknown mechanism which eliminates this cosmological constant from the observable Universe would also completely obliterate all other predictions based upon eq. (4.10). 
On the other hand, the physics of the low-energy moduli is locally rather than rigidly supersymmetric and thus the effective potential (4.10) is not positive definite. Hence, one can reasonably hope that for some theories there is at least an approximate cancellation between the positive and the negative terms in eq. (4.10) and the naive cosmological constant is therefore much smaller than $m_{3 / 2}^{2} M_{\mathrm{Pl}}^{2}$. For all the obvious reasons, this cannot be the final answer to the cosmological constant problem, but it at least makes it plausible that an unknown mechanism that does solve this problem does not do too much damage to the potential (4.10).

In both scenarios, the moduli masses are of the order $O\left(m_{3 / 2}\right)$, which may cause severe difficulties for the standard history of the early Universe. ${ }^{[5-57]}$ This problem is generic to all unified theories with moduli originating at the Planck scale that use the same field-theoretical mechanism for breaking SUSY and for stabilizing the moduli VEVs. One solution, suggested in ref. [57], involves not quite standard cosmology in which the inflation, the relaxation of the moduli scalars to their ultimate VEV and the baryogenesis all happen in the same era. Another solution is the hybrid scenario, in which one or more hidden sectors become strongly interacting at a rather high intermediate scale $\mu_{1}$ and stabilize all the moduli VEVs but do not break SUSY either directly or through the $W_{\bmod }(\Phi)$ and do not produce a cosmological constant. Then, at a much lower scale $\mu_{2}$, another hidden sector or sectors become strong and break SUSY. The hybrid scenario is rather baroque and we do not seriously advocate it as the ultimate answer to all the problems of the string unification, but it definitely warrants further investigation.

We conclude this article with a comment on how the two scenarios for SUSY breaking affect the observable sector of the low-energy theory. When the hidden sectors supply the $W_{\text {mod }}$ that triggers a spontaneous SUSY breakdown by the moduli superfields, it is the moduli dependence of the couplings $f_{a}^{W}, Y_{I J K}$ and $Z_{\bar{I} J}$ of the observable sector that breaks SUSY among the ordinary particles. Consequently, the superpartner masses are of the order $O\left(\left\langle F^{i}\right\rangle\right)=O\left(m_{3 / 2}\right)=O\left(M_{\bmod }\right)$; the detailed formulæ for the masses and other soft SUSY-breaking parameters are 
presented in ref. [26]. In this scenario, non-renormalizable (or even renormalizable) cross-couplings between the hidden and the observable sectors do not affect SUSY breaking. It is possible however that such cross-couplings may create an intermediate-energy supersymmetric threshold in the observable sector. Indeed, if some of the hidden matter fields are not quite hidden but have non-trivial charges under the observable gauge group, then the observable sector has a threshold near the confinement scale. Alternatively, a threshold may result from the Wilsonian superpotential of the EQFT containing a cross-coupling of the form $W \supset O\left(M_{\mathrm{Pl}}^{1-n}\right)\left(Q_{\mathrm{obs}}\right)^{2}\left(Q_{\mathrm{hid}}\right)^{n}$ (all indices suppressed). ${ }^{\star}$ Since the hidden sector does not break SUSY by itself, both kinds of thresholds are manifestly supersymmetric and their effects upon the Wilsonian gauge couplings of the observable sector are analytically computable with the help of eq. (3.28).

On the other hand, when the strong interactions in hidden sectors are directly responsible for the breakdown of SUSY, it is the moduli that play a peripheral role of merely determining the couplings of the EQFT whereas the SUSY breaking among the ordinary particles is controlled by the direct cross-couplings between the hidden and the observable sectors. One scenario of this kind involves matter fields that transform under both the observable gauge group and the hidden gauge group that breaks SUSY. In this case, SUSY breaking in the observable sector is caused by loops of gauge fields and charged particles and has nothing to do with the Planck-scale physics. A similar effect can be achieved by having gauge

* The energy scale of such a threshold depends on $n$ and on the kind of a hidden sector involved. When all of the hidden scalar VEVs are controlled by the renormalizable Yukawa couplings, one generally has $\left\langle\left(Q_{\text {hid }}\right)^{n}\right\rangle=O\left(\mu^{n}\right)$, which leads to the threshold scale $M_{I}=$ $O\left(m_{3 / 2}^{n / 3} M_{\mathrm{Pl}}^{1-n / 3}\right)$. In this scenario, the $n=1$ and $n=2$ cross-couplings produce thresholds well above the weak scale, the $n=3$ cross-couplings produce the so-called ' $\mu$-terms' right at the weak scale ${ }^{[58,26]}$ while the cross-couplings with $n \geq 4$ are too weak to have any physical effects. The situation is more complicated when it takes the non-renormalizable superpotential couplings to stabilize all the hidden VEVs. In this scenario, some of the hidden scalar VEVs are unusually large, of the order $\left\langle Q_{\text {hid }}\right\rangle=O\left(\mu^{1-\rho} M_{\mathrm{Pl}}^{\rho}\right)$ for some $0<$ $\rho<1$. The energy scale of the thresholds produced in this scenario depends not just on the $n$ but also on the particular hidden scalars participating in the cross-coupling. Generically, the observable sector may have a threshold above the weak scale whenever $n<3 /(1-\rho)$. 
singlets with Yukawa couplings to both the observable and the hidden sectors. ${ }^{[59]}$ Phenomenologically, this scenario makes for natural degeneracy among the squarks or sleptons of the same charge, which is good for avoiding the flavor-changing neutral currents. It also implies that the confinement scale of the SUSY-breaking sector is in the multi- TeV range and hence the gravitino and the moduli are very light, perhaps a fraction of an eV. Alternatively, SUSY breaking in the observable sector may be induced by the non-renormalizable cross-couplings to the hidden sector. We have not analyzed this scenario in any detail and can only say that the formulæ it yields are somewhat unwieldy.

Acknowledgements: Major parts of this work were performed at SLAC and CERN. We would like to take this opportunity to thank the members of both theory groups for their warm hospitality, their encouragement and for innumerable enlightening discussions we enjoyed over the past years. Our special thanks go to Brian Warr, for teaching us the fully covariant regularization techniques ( $c f$. Appendix B).

The research of V. K. is supported in part by the NSF, under grant PHY-9009850, and by the Robert A. Welch Foundation. The research of J. L. is supported by the Heisenberg Fellowship of the DFG. The collaboration of the two authors is additionally supported by NATO, under grant CRG 931380.

\section{APPENDIX A No-Renormalization Theorems for Locally supersymmetric EQFTs}

The key to the no-renormalization theorems is the locality of the Wilsonian renormalization group: Integrating out some high-momentum modes of a quantum field (or all the modes, if the field is heavy) adds to the Wilsonian action a spacetime-local term i.e., a $\int d^{4} x$ of a convergent power series in quantum fields and their derivatives that has no singularities at zero momentum. In a manifestly 
supersymmetric EQFT which is both quantized and cut-off in terms of explicit superfields, the renormalization of the Wilsonian action is local in superspace, that is, amounts to a full-superspace integral

$$
\Delta \mathcal{S}=\int d^{8} z \mathbf{E} \Delta \mathcal{L}
$$

of a non-singular function $\Delta \mathcal{L}$ of quantum and background superfields and their derivatives.

In the rigidly supersymmetric case, non-renormalization of the superpotential is an immediate consequence of the fact that a chiral-superspace integral of a derivative-less $\Delta W(\Phi, Q)$ cannot be re-written as a full-superspace integral (A.1) of a non-singular $\Delta \mathcal{L}^{[6]}$ In the locally supersymmetric case, the situation is somewhat different since the entire action (2.1) has a form of a full-superspace integral and there is no reason why the superspace-curvature superfields such as $\mathcal{R}$ or $\mathcal{W}_{\alpha \beta \gamma}$ cannot appear in $\Delta \mathcal{L}$ along with the other superfields of the theory. However, the locality of $\Delta \mathcal{L}$ restricts the manner of appearance of $\mathcal{R}$, etc., in $\Delta \mathcal{L}$ to expressions that do not lead to any singularities at zero curvature or zero derivatives of other fields. In particular, $\Delta \mathcal{L}$ cannot contain a $\Delta \widetilde{W} / 2 \mathcal{R}$ term with a derivative-less chiral $\Delta \widetilde{W}$ in the numerator and the curvature $\mathcal{R}$ in the denominator, and there are no other local non-singular full-superspace integrals whose flat-superspace limit is equivalent to a superpotential. Therefore, in locally supersymmetric EQFTs, just as in their rigidly supersymmetric analogues, the superpotential function $\widetilde{W}(\varphi, \Phi, Q)$ does not renormalize.

A similar argument reproduces the no-renormalization theorem for the gauge couplings $f_{a}$ : Following ref. [1] (where the rigidly supersymmetric case is treated), we quantize the gauge superfields in a background-covariant gauge and require the UV cutoff of the theory to be background-gauge invariant as well. Under these conditions, not only the integral (A.1) renormalizing the Wilsonian action should remain invariant under the background gauge transformations, but its integrand 
$\Delta \mathcal{L}$ itself should be background-gauge invariant. Alas, no full-superspace integral of a local, non-singular, gauge-invariant $\Delta \mathcal{L}$ has a component-field expansion containing $F_{m n}^{2}$. Consequently, the supersymmetric gauge couplings $\tilde{f}_{a}(\varphi, \Phi)$ appear to be renormalization-free.

As explained by Shifman and Vainshtein, this argument has a one-loop-hole: In background gauges, the normalization of matter, ghost and quantum vector superfields depends on the background. Consequently, integrating out the highmomentum modes of those superfields results in the anomalous renormalization of the action for the background gauge fields. From the path-integral point of view, this anomaly is the effect of the background-dependence of the measure for the modes being integrated out; in terms of the Feynman diagrams, this means that the entire anomaly is given by one-loop diagrams in which one of the background gauge vertices is replaced by a quantum vertex. ${ }^{[6]}$ Hence, the Wilsonian supersymmetric gauge couplings $\tilde{f}_{a}^{W}(\varphi, \Phi)$ do renormalize, but only at the one-loop level.

\section{APPENDIX B Regularizing a Locally Supersymmetric EQFT While Preserving All of its Symmetries}

The research that lead to this article began as a collaboration between Brian Warr and the two present authors. The results presented in this Appendix should be credited to Brian as much as to V. K. and J. L. combined; alas, Brian's untimely death prevented him from further participation in this project. The two of us would like to use this opportunity to express our deep sorrow at his passing and to acknowledge the work he has done.

Many formal arguments about supersymmetric gauge theories presume the existence of an ultraviolet regularization scheme that preserves the manifest supersymmetry of the superfield formalism and at the same time has manifest fourdimensional background gauge invariance and a BRST symmetry protecting the 
quantum gauge invariance. In refs. [60], Brian Warr proved that there indeed exists such a regularization scheme*. Specifically, he showed that for the non-chiral supersymmetric gauge theories, ${ }^{\dagger}$ it is possible to combine a huge-but-finite set of Pauli-Villars-like compensating superfields and a carefully chosen pattern of fullycovariant-higher-derivative propagator-regulating terms in the Lagrangian and thus regularize all divergencies of the perturbation theory. The manifest covariance of this regularization scheme is plain to see; the difficult part of the Warr's proof was to show that it does regularize all the perturbative divergences. Regularizing all the divergences of a chiral gauge theory is more difficult, and thus the chiral version of the Warr's regularization scheme (WRS) is not quite manifestly gauge invariant; nevertheless, this scheme is sufficient to prove all the Ward identities of a chiral gauge symmetry that is free of the triangle and linear (trace) anomalies.

\section{B.1. Rigidly Supersymmetric WarR's Scheme.}

Before we proceed with adapting the WRS to locally supersymmetric EQFTs, let us review its rigidly supersymmetric version. To be precise, we present a superfield version of the non-chiral WRS which is manifestly invariant under the background gauge transformations and regularizes all the divergences of a nonchiral gauge theory; we shall briefly discuss the chiral case later in this section. The formalism of this Appendix and of the following Appendix $\mathrm{C}$ is based upon ref. [6], which also describes the supersymmetric quantization procedure.

Our first step towards constructing the WRS is to soften the UV behavior of the matter superfields' propagators. For this purpose, we add to the bare action

$\star$ For the discussion of alternative regularization schemes see refs. [61,6].

$\dagger$ A gauge theory, supersymmetric or otherwise, is called chiral or non-chiral according to whether the left-handed Weyl fermions together form a complex or a self-conjugate representation of the gauge group. For a supersymmetric gauge theory, this criterion has nothing to do with the chirality of the matter superfields $Q^{I}$ — they are always chiral and everything to do with their gauge quantum numbers. 
of the theory covariant higher derivative operators that generically look like

$$
A^{(n)} \int d^{4} x d^{4} \Theta \bar{Q} e^{2 V} \square^{n} Q
$$

where $n$ is a positive integer, the normalization factor $A^{(n)}$ is a real constant of the order $O\left(\Lambda^{-2 n}\right)$, all indices are suppressed and

$$
\square=\frac{1}{16} \bar{D}^{2} e^{-2 V} D^{2} e^{+2 V}
$$

is the covariant D'Alambertian for the charged chiral superfields. The name "covariant D'Alambertian" means that $\square Q$ is a chiral superfield that transforms under the gauge transformations exactly like the $Q$, but in the trivial gauge background $V \equiv 0, \square Q$ reduces to simply $\square Q \equiv \partial^{\mu} \partial_{\mu} Q$. The covariance of the operator $\square$ provides for the gauge invariance of the regulators (B.1) while its D'Alambertian nature tells us the effect of those regulators on the scalar propagators of the theory. For a massless chiral superfield, the regularized superspace propagator is

$$
\frac{\frac{1}{16} D^{2} \bar{D}^{2} \delta^{(4)}(\Theta)}{p^{2} A\left(p^{2}\right)} \equiv \frac{\frac{1}{16} D^{2} \bar{D}^{2} \delta^{(4)}(\Theta)}{p^{2}\left(1+A^{(1)} p^{2}+A^{(2)} p^{4}+\cdots\right)}
$$

while for a massive scalar supermultiplet, the four $(Q, \bar{Q}) \mapsto(\bar{Q}, Q)$ propagators can be summarized in a two-by-two matrix

$$
\frac{1}{|M|^{2}+p^{2} A^{2}\left(p^{2}\right)} \times\left(\begin{array}{cc}
A\left(p^{2}\right)\left(\frac{1}{16} D^{2} \bar{D}^{2}\right) & M\left(\frac{1}{4} D^{2}\right) \\
M^{*}\left(\frac{1}{4} \bar{D}^{2}\right) & A\left(p^{2}\right)\left(\frac{1}{16} \bar{D}^{2} D^{2}\right)
\end{array}\right) \delta^{(4)}(\Theta) .
$$

The ultraviolet limits of these propagators are controlled by the regulator (B.1) with the largest number of derivatives, i.e., with the largest $n$. Specifically, apart from the $D^{2}$ and the $\bar{D}^{2}$ factors, the propagators (B.3) and (B.4) decrease with $|p| \gtrsim \Lambda$ like $\left(1 / p^{2}\right)^{n_{\max }+1}$, in obvious notations; we shall refer to the $n_{\max }$ as the type of the matter superfield. 
Similarly, we soften the UV behavior of the gauge superfields' propagators by adding to the bare action of the theory covariant higher derivative operators

$$
\frac{1}{4} B^{(n)} \int d^{4} x d^{2} \Theta W^{\alpha} \square^{n} W_{\alpha}+\text { H.c. }
$$

(gauge indices suppressed). Again, the $\square$ here is the covariant D'Alambertian (B.2) for the chiral, gauge-covariant superfields (such as $W^{\alpha}$ ); this assures that the terms (B.5) are both gauge-invariant and supersymmetric. The normalization factors $B^{(n)}$ are constants of the order $O\left(\Lambda^{-2 n}\right)$; for simplicity, we assume real $B^{(n)}$. The precise effect of the regularizing terms (B.5) on the gauge superfield's propagator depend on the gauge-fixing scheme; in the super-Feynman gauge, the propagator is

$$
\frac{\delta^{(4)}(\Theta)}{(\operatorname{Re} f) p^{2}+B^{(1)} p^{4}+B^{(2)} p^{6}+\cdots}
$$

while in the 'super-transverse' gauge favored by Warr the propagator is somewhat more complicated.

The gauge-invariance of the higher-derivative propagator-regularizing terms has its price, namely the higher-derivative couplings of the gauge superfields to themselves and to the charged scalar superfields; these couplings tend to counteract the regularizing effect of the propagator softening. Careful counting of powers of all the momenta involved in a generic superfield Feynman diagram (including the momenta implicit in the $D$ operators) shows that all the multi-loop diagrams are superficially convergent as long as the gauge superfields are of a higher type than any scalar superfield, i.e., $n_{\max }$ (gauge) $>n_{\max }$ (any scalar) ${ }^{[60]}$ (If there are Yukawa couplings between the matter superfields, one also needs $n_{\max }$ (matter) $\geq \frac{1}{3} n_{\max }$ (gauge).) Some of the one-loop diagrams however remain superficially divergent in spite of the covariant-higher-derivative regulators and thus must be regularized in some other manner, both for their own sake and to eliminate possible sub-diagram divergencies of the multi-loop diagrams. 
A divergent one-loop diagram (or sub-diagram) may have an arbitrary number of external gauge legs but no external legs belonging to matter or ghost superfields. For non-chiral gauge theories, we may calculate the one-loop diagrams using the second order formalism (see Appendix C for details), which automatically avoids any quadratic or linear divergences while the logarithmic divergences consistently assemble into infinite renormalization of the gauge coupling. To cancel these logarithmic divergences, we add to the theory an array of Pauli-Villars superfields $(\mathrm{PVs})$, which are heavy (mass of the order $O(\Lambda)$ ) scalar superfields with covariant higher-derivative Lagrangians; generically, we need several kinds of PVs with different gauge quantum numbers, different types and both Bose and Fermi statistics. The complete spectrum of the PVs should satisfy the equations

$$
\begin{aligned}
\sum_{\mathcal{P}}^{\mathrm{PV}} & \pm T_{a}(\mathcal{P})\left(2 n_{\max }(\mathcal{P})+1\right) \\
& =T\left(G_{a}\right)\left(2 n_{\max }(\text { gauge })+3\right)-\sum_{r} n_{r}(\text { matter }) T_{a}(r)\left(2 n_{\max }(\text { matter })+1\right)
\end{aligned}
$$

for all gauge group factors $G_{a}$; in our notations, $\pm T_{a}(\mathcal{P})$ refers to the statistics and to the gauge quantum numbers of the Pauli-Villars superfield $\mathcal{P}$. The details of the solution of all the eqs. (B.7) are not germane to the subject matter of this article; all we really need to know is that the solution exists.

Regularizing all the one-loop divergences of a chiral gauge theory is more difficult since the second order formalism does not work for the chiral superfields in a complex representation of the gauge group. The first order formalism is plagued by the quadratic and linear divergences that must be pre-regularized before one can put together Feynman diagrams with different arrangements of external legs. In the chiral WRS, the pre-regularization is achieved with the help of additional PauliVillars superfields of mass $M_{\text {pre }} \gg \Lambda$. The pre-regularizing PVs form complex representations of the gauge group; their higher-derivative kinetic energy terms are gauge-covariant, but their mass terms are not. The gauge invariance of the 
effective theory is restored by adding a local counter-term to the bare action of the theory; this is possible whenever the theory is free from the triangle and linear (trace) anomalies. The gauge invariance of the chiral WRS is thus not quite manifest; nevertheless, thanks to $M_{\text {pre }} \gg \Lambda$, the gauge symmetry is completely restored at energies well above the ordinary UV regularization scale $\Lambda$ and the exact form of the counter-term is completely determined at the one-loop level of the theory. Therefore, as far as the Ward identities - and their corollaries - are concerned, the chiral WRS is as good as if it was manifestly gauge invariant.

We close this overview by stressing that the WRS regularizes the UV divergencies of Feynman diagrams and has nothing to do with the convergence/divergence of the perturbative expansion of the EQFT. Moreover, the finite theory defined by the WRS is unitary only in the limit of the UV cutoff $\Lambda$ being much bigger then the momenta of any external particles involved in the S-matrix; when the external momenta become comparable with the cutoff, the unitarity breaks down. In short, the WRS is not a supersymmetric substitute for a lattice regularization, and we do not propose a locally supersymmetric substitute for a lattice either. Instead, we are about to construct a manifestly supersymmetric and gauge invariant regularization scheme for locally supersymmetric EQFTs whose UV limits are perturbative (as long as the supergravity itself is limited to the background).

\section{B.2. WARR's SCHEME FOR LOCALly-Supersymmetric EQFTs.}

Adapting the WRS to locally supersymmetric EQFTs involves two tasks: First, we modify the regularizing terms in the action to make them manifestly invariant under the local SUSY, which in our formalism comprises the general supercoordinate, local Lorentz and super-Weyl transformations. In addition, we would also like to have manifest background gauge symmetry of the entire action; therefore, we will use the background-covariant version of the super-Feynman gauge. The second task is to regularize the divergencies that involve non-trivial supergravitational or moduli backgrounds; this work is still in progress, so we shall 
present only the results which are relevant to the calculations of the Appendix C.

We begin with fully-covariant derivatives for the chiral superfields. When acting upon spinless, Weyl invariant superfields, the operators

$$
\varphi^{-2}\left(\frac{1}{4} \overline{\mathcal{D}}^{2}-2 \mathcal{R}\right) \bar{\varphi} \quad \text { and } \quad \bar{\varphi}^{-2}\left(\frac{1}{4} \mathcal{D}^{2}-2 \overline{\mathcal{R}}\right) \varphi
$$

are super-covariant as well as Lorentz and Weyl invariant (albeit not gauge-covariant). Hence, for a charged but spinless and Weyl invariant chiral superfield such as $Q$, the fully-covariant D'Alambertian can be written as

$$
\triangle=\frac{1}{\varphi^{2}}\left(\frac{1}{4} \overline{\mathcal{D}}^{2}-2 \mathcal{R}\right) \frac{1}{\bar{\varphi}} e^{-2 V}\left(\frac{1}{4} \mathcal{D}^{2}-2 \overline{\mathcal{R}}\right) \varphi e^{+2 V} .
$$

By full covariance we mean that $\triangle Q$ transforms exactly like $Q$ under all the local symmetries of the theory, namely gauge, SUSY, Lorentz and super-Weyl. Given this fully-covariant D'Alambertian, we can write the fully-symmetric analogues of the matter-propagator regulating terms (B.1) as

$$
\int d^{8} z \mathbf{E} \varphi \bar{\varphi} A_{\bar{I} J}^{(n)} \bar{Q}^{\bar{I}} e^{2 V} \triangle^{n} Q^{J}
$$

Generically, the matrices $A_{\bar{I} J}^{(n)}$ have the same structure as the $Z_{\bar{I} J}$ matrix, but without any loss of regularizing power we may choose to have $A_{\bar{I} J}^{(n)}=A^{(n)} \delta_{\bar{I} J}$ where the overall coefficient $A^{(n)}=O\left(\Lambda^{-2 n}\right), \Lambda$ being the scale parameter of the UV cutoff. In any case, the matrix elements $A_{\bar{I} J}^{(n)}$ must be $\varphi$ independent and it is best to have them moduli-independent as well.

The fully-symmetric analogues of the gauge-propagator regulating operators (B.5) can be written in a similar fashion, namely

$$
\frac{1}{4} B^{(n)} \int d^{8} z \mathcal{E} \mathcal{W}^{\alpha}\left(\varphi^{3 / 2} \triangle \varphi^{-3 / 2}\right)^{n} \mathcal{W}_{\alpha}+\text { H.c. }
$$

where $\varphi^{3 / 2} \triangle \varphi^{-3 / 2}$ is the fully-covariant D'Alambertian for the gauge field stress tensor $\mathcal{W}^{\alpha}$ whose Weyl weight is 3. Generically, one may have different coefficient 
$B_{a}^{(n)}$ for the different irreducible factors $a$ of the gauge group, but without any loss of regularizing power we may choose the same $B^{(n)}=O\left(\Lambda^{-2 n}\right)$ for all $a$. In any case, all the $B_{a}^{(n)}$ must be $\varphi$ independent and it is best to have them moduliindependent as well.

So far we have described the minimal covariantization of the higher derivative regularizing operators with respect to the local SUSY. To these minimal operators (B.10) and (B.11) we may (and should) add non-minimal operators that involve covariant superspace curvature tensors such as $\mathcal{W}^{\alpha \beta \gamma}$ or $\mathcal{R}-\frac{1}{8} \overline{\mathcal{D}}^{2} \log \bar{\varphi}$. These non-minimal higher derivative operators play an important role in regularizing Feynman diagrams with gravitational external lines.

Now consider the background-covariant super-Feynman gauge for locally supersymmetric gauge theories. As in the rigid case, ${ }^{[6]}$ we split the gauge superfield $V$ into the quantum and the background gauge superfields according to

$$
\exp (2 V)=\exp \left(\Omega^{\dagger}\right) \exp (2 \mathbf{v}) \exp (\Omega)
$$

The quantum gauge superfield $\mathbf{v}$ is hermitian; the background gauge superfield $\Omega$ is neither hermitian nor chiral. The locally-supersymmetric, background-covariant analogues of the super-Landau gauge conditions can be written as

$$
\begin{gathered}
{\left[\varphi^{w-2}\left(\frac{1}{4} \overline{\mathcal{D}}^{2}-2 \mathcal{R}\right) \bar{\varphi}\left(e^{-\Omega}\right)_{(b)}^{(a)}\right] \mathbf{v}^{(b)}=\mathbf{G}^{(a)},} \\
{\left[\bar{\varphi}^{w-2}\left(\frac{1}{4} \mathcal{D}^{2}-2 \overline{\mathcal{R}}\right) \varphi\left(e^{\Omega^{\dagger}}\right)_{(b)}^{(a)}\right] \mathbf{v}^{(b)}=\overline{\mathbf{G}}^{(a)},}
\end{gathered}
$$

where $\mathbf{G}^{(a)}$ and $\overline{\mathbf{G}}^{(a)}$ are fixed but arbitrary chiral and anti-chiral superfields. Eqs. (B.13) fix the quantum gauge symmetry of $\mathbf{v}$, but they are covariant with respect to the background gauge symmetry, under which $\mathbf{G}$ and $\overline{\mathbf{G}}$ behave like ordinary adjoint multiplets of scalar superfields. Generically, the Weyl weight of the superfields $\mathbf{G}$ and $\overline{\mathbf{G}}$ is an arbitrary real parameter $w$ of the gauge-fixing 
conditions (B.13), but in order to simplify the ghost structure of the EQFT (see below), we prefer to use $w=3$.

The Faddeev-Popov ghost action corresponding to the gauge conditions (B.13) follows from the general rules ${ }^{[6]}$ :

$$
\begin{aligned}
\mathcal{S}_{\mathrm{FP}}=\int d^{8} z \mathcal{E} c^{\prime}\left[\varphi^{w-2}\left(\frac{1}{4} \overline{\mathcal{D}}^{2}-2 \mathcal{R}\right) \bar{\varphi} e^{-\Omega}\right]\left(\frac{\partial V}{\partial \xi} c+\frac{\partial V}{\partial \bar{\xi}} \bar{c}\right)+\text { H. c. } \\
=\int d^{8} z \mathbf{E}\left(\varphi^{w-2} \bar{\varphi} c^{\prime} e^{-\Omega}+\varphi \bar{\varphi}^{w-2} \bar{c}^{\prime} e^{+\Omega^{\dagger}}\right) \\
{\left[\mathbf{v}\left(e^{+\Omega} c+e^{-\Omega^{\dagger}} \bar{c}\right)+\frac{\mathbf{v}}{\tanh \mathbf{v}}\left(e^{+\Omega} c-e^{-\Omega^{\dagger} \bar{c}}\right)\right] }
\end{aligned}
$$

(gauge indices suppressed). The ghosts $c$ and $\bar{c}$ have the same quantum numbers as the parameters $\xi$ and $\bar{\xi}$ of infinitesimal quantum gauge transforms: They are chiral/antichiral Weyl-invariant superfields in the adjoint representation of the background gauge group. The anti-ghosts $c^{\prime}$ and $\bar{c}^{\prime}$ are also chiral/antichiral superfields in the adjoint representation of the background gauge group, but their Weyl weights are $(3-w)$. For $w=3$, all Faddeev-Popov ghosts are Weyl invariant and the ghost action (B.14) becomes

$$
\mathcal{S}_{\mathrm{FP}}=\int d^{8} z \mathbf{E} \varphi \bar{\varphi}\left(\bar{c}^{\prime} e^{\Omega^{\dagger}} e^{\Omega} c+\bar{c} e^{\Omega^{\dagger}} e^{\Omega} c^{\prime}+c^{\prime} c-\bar{c}^{\prime} \bar{c}+\mathcal{L}_{\mathrm{int}}\left(c, c^{\prime}, \bar{c}, \bar{c}^{\prime}, \mathbf{v}, \Omega, \Omega^{\dagger}\right)\right) .
$$

Apart from the overall factor $\mathbf{E} \varphi \bar{\varphi}$, this ghost Lagrangian is identical to its rigid counterpart; furthermore, the Faddeev-Popov ghosts are of type zero - they do not have any higher-derivative terms in their Lagrangian.

In Feynman gauges, one averages the path integral over the Landau gauge parameters $\mathbf{G}^{(a)}$ and $\overline{\mathbf{G}}^{(a)}$ with a Gaussian weight; this is equivalent to replacing the constraints (B.13) with a gauge-fixing action term. To simplify the gauge superfields' propagators, the gauge-fixing action should contain background-covariant higher-derivative terms that exactly parallel the higher-derivative terms in the 
gauge-invariant action. Thus, in matrix notations,

$$
\mathcal{S}_{G F}=\int d^{8} z \mathbf{E}(\varphi \bar{\varphi})^{-2} \overline{\mathbf{G}} e^{\Omega^{\dagger}} e^{\Omega}\left[(\operatorname{Re} f)+\sum_{n=1}^{n_{\max }(\text { gauge })} B^{(n)}\left(-\varphi^{3} \triangle(\mathrm{bg}) \varphi^{-3}\right)^{n}\right] \mathbf{G},
$$

where $f$ is the matrix of the bare gauge couplings $f_{(a)(b)}=f_{a} \delta_{(a)(b)}$ of the unregularized EQFT; in the manifestly super-Weyl invariant formalism of this Appendix, $f$ does not depend on the $\varphi$. The coefficients $B^{(n)}$ in eq. (B.16) are exactly as in eq. (B.11) and the background-covariant $\triangle(\mathrm{bg})$ is obtained from the fullycovariant $\triangle$ via disregarding the quantum gauge superfield $\mathbf{v}$, i.e., via replacing $e^{2 V}$ with $e^{\Omega^{\dagger}} e^{\Omega}$; the peculiar powers of $\varphi$ and $\bar{\varphi}$ in eq. (B.16) correspond to $w=3$. To properly compensate for the gauge averaging, we should add to the theory the Nielsen-Kallosh ghosts $b^{(a)}$ and $\bar{b}^{(a)}$, whose action mirrors (B.16):

$$
\mathcal{S}_{N K}=\int d^{8} z \mathbf{E}(\varphi \bar{\varphi})^{-2} \bar{b} e^{\Omega^{\dagger}} e^{\Omega}\left[(\operatorname{Re} f)+\sum_{n=1}^{n_{\max }(\text { gauge })} B^{(n)}\left(-\varphi^{3} \triangle(\mathrm{bg}) \varphi^{-3}\right)^{n}\right] b ;
$$

as usual, the Nielsen-Kallosh ghosts do not couple to the quantum gauge superfields $\mathbf{v}^{(a)}$ or to any other quantum fields, but they are sensitive to $\Omega, \Omega^{\dagger}$ and other background fields. Similar to the Faddeev-Popov ghosts and antighosts, Nielsen-Kallosh ghosts are fermionic spinless (anti) chiral superfields in the adjoint representation of the background gauge group. Unlike the Faddeev-Popov ghosts, the Nielsen-Kallosh ghosts have non-zero Weyl weight $w=3$ and type $n_{\max }(b, \bar{b})=n_{\max }($ gauge $)$.

Finally, consider the Pauli-Villars superfields $\mathcal{P}$ of a locally supersymmetric EQFT. In the non-chiral case when $Q^{I}$ together form a self-conjugate representation of the gauge group, we need only the PVs that are massive scalar superfields with fully covariant actions

$$
\begin{aligned}
\int d^{8} z \mathbf{E}(\varphi \bar{\varphi})^{1-w} & \overline{\mathcal{P}}\left[\sum_{n=0}^{n_{\max }} A_{\mathcal{P}}^{(n)} e^{2 V}\left(-\varphi^{w} \triangle \varphi^{-w}\right)^{n}\right] \mathcal{P} \\
& +\int d^{8} z \mathcal{E} \frac{1}{2} \varphi^{3-2 w} M_{\mathcal{P}} \mathcal{P}^{2}+\text { H.c. }
\end{aligned}
$$


where $A_{\mathcal{P}}^{(n)}=O\left(\Lambda^{-2 n}\right), M_{\mathcal{P}}=O(\Lambda)$ and neither $A_{\mathcal{P}}^{(n)}$ nor $M_{\mathcal{P}}$ depends on either $\varphi$ or the moduli of the EQFT. Different Pauli-Villars superfields may have different types $n_{\max }$ and different Weyl weights $w$ as well as different statistics and gauge-group quantum numbers. In addition, similar to the matter and the gauge superfields, the PVs may have non-minimal higher-derivative couplings to the covariant superspace curvature tensors.

As in the rigid case, the spectrum of the PVs must satisfy equations (B.7) in order to cancel all the divergences that do not involve external gravitational, $\varphi$ or moduli superfields. Cancelation of the divergences that do involve superspace curvatures or derivatives of the super-Weyl compensators imposes additional equations similar to eq. (B.7) ${ }^{\star}$; it is these additional equations that determine the Weyl weights of the PVs and the coefficients of the non-minimal higher-derivative operators for both the PVs and the matter and the gauge superfields. On the other hand, any possible divergences involving the moduli fields are completely regularized by the higher-derivative regulators and impose no constraints on the PVs; this follows from the moduli independence of the higher-derivative coefficients $A^{(n)}, B^{(n)}$ and $A_{\mathcal{P}}^{(n)}$.

For locally-supersymmetric EQFTs with complex spectra of the matter superfields $Q^{I}$, we need additional, heavier PVs to pre-regularize all the one-loop divergences of the first order formalism. We have not worked out all the details of the locally-supersymmetric pre-regularization; however, by analogy with the rigid case, we believe that complete pre-regularization of the one-loop divergences can be achieved using PVs with fully-covariant kinetic and higher-derivative actions but with masses that break gauge invariance.

\footnotetext{
* Actually, all multi-loop Feynman diagrams, with or without gravitational or $\varphi$ external lines, are superficially regularized by the minimal higher-derivative operators alone. It is the one-loop diagrams that impose constraints on the spectrum of the PVs. In this aspect, locally supersymmetric EQFTs do not differ from their rigid analogues.
} 


\section{APPENDIX C \\ Gauge Couplings of a Locally Supersymmetric EQFT: Explicit Superfield Calculations of the One Loop Effects.}

In Appendix B, we described a regularization scheme that is manifestly symmetric with respect to both local SUSY (including manifest super-Weyl symmetry) and background gauge symmetry. In this Appendix, we use this scheme to explicitly calculate to the one-loop order the differences between the bare, the Wilsonian and the effective gauge couplings; as promised, our results agree with eqs. (2.20), (2.22), (3.4) and (3.7). Whereas the super-Weyl anomaly responsible for the Kähler terms in eqs. (2.20) and (3.7) is exhausted at the one loop level, the calculations of this Appendix effectively confirm those equations to all orders of the perturbation theory.

The effective couplings are properties of the generating functional of the EQFT; in a background-gauge covariant formalism, the effective gauge couplings are given by the two-point Green's functions for the background gauge fields; the superspace analogue of eq. (3.1) is

$$
\mathcal{A}\left(W^{(a) \alpha}(-p), W_{\alpha}^{(a)}(p)\right)=\frac{-4}{\bar{D}^{2}} \frac{1}{\left\{g_{a}(p)\right\}^{2}} .
$$

The two-point Green's function (C.1) is even and, at the one-loop level of analysis, it does not distinguish whether the matter superfields form a representation $r$ of the gauge group or a complex conjugate representation $\bar{r}$. Therefore, once we derive a general formula for the $\left\{g_{a}\right\}$ of an EQFT with a non-chiral spectrum of the chiral matter superfields $Q^{I}$, the same formula should apply verbatim to the chiral EQFTs as well. In light of this observation, we shall limit our explicit calculations to the non chiral case and thus avoid the pre-regulation and all the associated difficulties.

At the one-loop level of analysis, the entire generating functional for all the background fields is the logarithm of the super-determinant of the background- 
dependent matrix of second variational derivatives of the EQFT's bare Lagrangian with respect to all the quantum superfields of the theory. Since we are interested only in the gauge couplings and their moduli and $\varphi$ dependence, we presume a gravitationally trivial background (i.e., flat vielbein $E_{M}^{A}$ ) while allowing arbitrary configurations of the background gauge superfields. Similarly, we presume the moduli superfields and the $\varphi$ to have uniform values throughout the superspace, although we make no assumptions about the actual values of the $\left\langle\Phi^{i}\right\rangle$ and the $\langle\varphi\rangle$. These assumptions effectively reduce a locally-supersymmetric EQFT with moduli fields to a rigidly-supersymmetric EQFT with adjustable couplings and regularizing terms. For example, fully-covariant higher-derivative regulator terms (B.10) reduce to (B.1), except that the coefficients $A^{(n)}$ become $\langle\varphi\rangle$-dependent $\tilde{A}^{(n)}=|\langle\varphi\rangle|^{2-2 n} A^{(n)}$. Note however that the un-rescaled Minkowski metric of the effective rigid theory is not the physical Einstein metric of the locally supersymmetric theory; we shall return to this point later in this section.

In the background-covariant super-Feynman gauge, the quantum gauge superfields $\mathbf{v}^{(a)}$ are full (off-shell) vector superfields and, according to the standard lore ${ }^{[6]}$ their loops do not contribute to the $\left\{g_{a}^{-2}\right\}^{1-\text { loop }}$. Ref. [6] does not consider the higher-derivative terms (B.11), but the argument works even when such terms are present. Indeed, let us take the second variational derivative $\delta^{2} / \delta \mathbf{v}^{2}$ of the entire bare Lagrangian of the EQFT, including the higher-derivative terms (B.11) and the gauge-fixing terms (B.16). The result is a background-gauge covariant operator, which, after some algebra, can be written as a polynomial of degree $n_{\max }$ (gauge) +1 in operator products $\eta_{a b} \nabla^{a} \nabla^{b}, W_{\mathrm{bg}}^{\alpha} \nabla_{\alpha}$ and $\bar{W}_{\mathrm{bg}}^{\dot{\alpha}} \nabla_{\dot{\alpha}}$ $\left(\nabla_{\dot{\alpha}} \equiv e^{+\Omega} \bar{D}_{\dot{\alpha}} e^{-\Omega}, \nabla_{\alpha} \equiv e^{-\Omega^{\dagger}} D_{\alpha} e^{+\Omega^{\dagger}}\right.$ and $\left.\nabla^{a} \equiv \frac{1}{4} \sigma_{\alpha \dot{\alpha}}^{a}\left\{\nabla^{\alpha}, \nabla^{\dot{\alpha}}\right\}\right)$. There also are terms involving higher covariant derivatives of the background gauge fields, but all such terms are suppressed by negative powers of the UV cutoff scale $\Lambda$ and have negligible effects on Green's functions of gauge particles with momenta $p \ll \Lambda$. The remaining terms have at most one spinorial derivative $\nabla^{\alpha}$ or $\nabla^{\dot{\alpha}}$ acting upon the quantum gauge superfield $\mathbf{v}$ for each power of a background gauge connection 
or curvature; consequently, any one-loop Feynman diagram with the v superfield running around the loop has at most one spinorial derivative in the loop per external leg. According to the superfield Feynman rules, a loop integral vanishes unless the loop contains at least four spinorial derivatives; therefore, in the backgroundcovariant Feynman gauge, the one-loop diagrams with the $\mathbf{v}$ loop do not contribute to the two-point and three-point Green's functions for the background gauge fields.

Besides the $\mathbf{v}^{(a)}$, all other quantum superfields of the EQFT - the matter superfields, the ghosts and the PVs - are chiral and their respective contributions to the $\left\{g_{a}^{-2}\right\}^{1-\text { loop }}$ can be computed in similar ways. In flat superspace, contribution of a generic chiral superfield $C$ to the one-loop generating functional of the background fields can be formally written as

$$
\pm \operatorname{Sdet}\left(\begin{array}{ll}
\frac{\delta^{2} \mathcal{L}_{+}}{C^{2}} & \frac{\delta^{2} \mathcal{L}_{-}}{C \bar{C}} \\
\frac{\delta^{2} \mathcal{L}_{+}}{\bar{C} C} & \frac{\delta^{2} \mathcal{L}_{-}}{\bar{C}^{2}}
\end{array}\right)
$$

where the overall sign \pm depends on the statistics of $C$ and the $\mathcal{L}_{+}$and the $\mathcal{L}_{-}$ are respectively the chiral- and the antichiral-superspace Lagrangians, i.e.,

$$
\mathcal{S}=\int d^{4} x d^{2} \Theta \mathcal{L}_{+}=\int d^{4} x d^{2} \bar{\Theta} \mathcal{L}_{-}
$$

For a chiral superfield $C$ of type $n_{\max }$ in a background of constant moduli superfields and $\varphi$, we have

$$
\begin{aligned}
\frac{\delta^{2} \mathcal{L}_{+}}{C^{2}} & =\widetilde{M}, \quad \frac{\delta^{2} \mathcal{L}_{-}}{\bar{C}^{2}}=\widetilde{M}^{*} \\
\frac{\delta^{2} \mathcal{L}_{+}}{\bar{C} C} & =\frac{1}{4} D^{2} e^{2 V} A(-\square), \\
\frac{\delta^{2} \mathcal{L}_{-}}{C \bar{C}} & =\frac{1}{4} \bar{D}^{2}\left(e^{2 V} A(-\square)\right)^{\top}=A(-\square) \bar{D}^{2} e^{-2 V},
\end{aligned}
$$

where $\widetilde{M}=\langle\varphi\rangle^{3-2 w} M$ is the un-normalized, $\varphi$-dependent bare mass of $C, A$ is a polynomial of degree $n_{\max }$ with $\varphi$-dependent coefficients $\tilde{A}^{(n)}=|\langle\varphi\rangle|^{2-2 n-2 w} A^{(n)}$ 
$\left(n=0,1, \ldots, n_{\max }\right)$ and $V$ is the background gauge superfield, $e^{2 V}=e^{\Omega^{\dagger}} e^{\Omega}$; the second equality in the last equation (C.4) is valid for self-conjugate representations of the background gauge group. Thus, for non-chiral gauge theories, we substitute eqs. (C.4) into eq. (C.2), simplify the determinant and arrive at

$$
\pm \operatorname{Sdet}\left(|\widetilde{M}|^{2}-A(-\square) \square A(-\square)\right)= \pm \operatorname{Sdet}(H(-\square))
$$

where $H$ is a polynomial defined according to $H(x)=|\widetilde{M}|^{2}+x A^{2}(x)$.

Eq. (C.5) defines fairly simple second-order Feynman rules for the charged chiral superfields: The propagator of the scalar superfield $C$ is $\delta^{(4)}(\Theta) / H\left(k^{2}\right)$ while the vertices that come with one spinorial derivative $D_{\alpha}$ in the loop per external gauge line are $H^{\prime}\left(k^{2}\right) W_{\mathrm{bg}}^{\alpha} D_{\alpha}$ and $\frac{1}{2} H^{\prime \prime}\left(k^{2}\right)\left(W_{\mathrm{bg}}^{\alpha} D_{\alpha}\right)^{2}$; there are other kinds of vertices, but they carry fewer $D_{\alpha}$ 's and thus do not contribute to the two-point functions. As described in ref. [6], one of the external gauge lines connected to the loop comes with an additional factor $\bar{D}^{2}$ in the loop at the expense of a similar factor on the external line; this is precisely the mechanism that gives rise to the two-point functions of the form (C.1).

Topologically, there are two one-loop Feynman diagrams with two external legs:
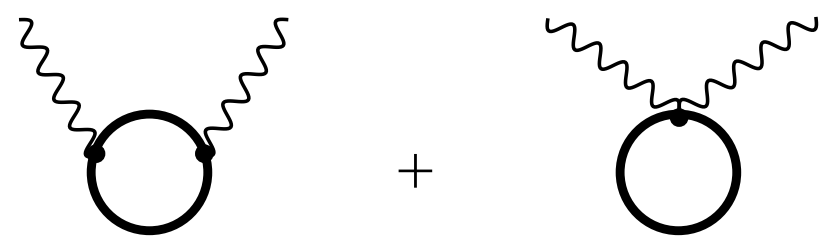

The $d^{4} \Theta$ integrals associated with the two diagrams are very simple; generically, both diagrams contribute to the effective gauge couplings and the respective contributions are

$$
\pm T_{a} \int \frac{d^{4} k}{(2 \pi)^{4}} \frac{\left(H^{\prime}\left(k^{2}\right)\right)^{2}}{H\left(k^{2}\right) H\left((k+p)^{2}\right)} \quad \text { and } \quad-\left( \pm T_{a}\right) \int \frac{d^{4} k}{(2 \pi)^{4}} \frac{H^{\prime \prime}\left(k^{2}\right)}{H\left(k^{2}\right)} .
$$

In these formulæ, $\pm T_{a}$ refers to the statistics and the gauge quantum numbers of 
the chiral superfield that runs around the loop, $k$ is the loop momentum and $p$ is the momentum of the external legs; we presume $p$ to be much smaller than the UV cutoff scale $\Lambda$ while $A^{(n)}=O\left(\Lambda^{-2 n}\right)$. Evaluating the Feynman integrals (C.7) shows that their sum does not depend on the details of the polynomial $H\left(k^{2}\right)$ but only on its limiting behaviors for $k^{2} \rightarrow \infty$ and for $k=O(|p|) \ll \Lambda$. The result is particularly simple for a chiral superfield that is either much heavier or much lighter than $p$ : for $|\widetilde{M}|^{2} \gg p^{2}$, the two integrals (C.7) total to

$$
\frac{ \pm T_{a}}{16 \pi^{2}}\left(\left(2 n_{\max }+1\right)\left(\log k_{\max }^{2}-1\right)+2 \log \frac{\tilde{A}^{\left(n_{\max }\right)}}{|\widetilde{M}|}\right)
$$

while for $\widetilde{M}=0$ or $|\widetilde{M}|^{2} \ll p^{2}$, the result is

$$
\frac{ \pm T_{a}}{16 \pi^{2}}\left(\left(2 n_{\max }+1\right)\left(\log k_{\max }^{2}-1\right)+2 \log \frac{\tilde{A}^{\left(n_{\max }\right)}}{\tilde{A}^{(0)}}-\log p^{2}+3\right)
$$

The appearance of the $\log k_{\max }^{2}$ terms in these formulæ reflects the logarithmic UV divergence of the individual superfields' contributions. However, once we sum over all the superfields that may run around the loop, the divergences cancel out. Indeed, eqs. (B.7) for the types, statistics and gauge quantum numbers of the Pauli-Villars superfields are equivalent to

$$
\sum_{C} \pm T_{a}(C)\left(2 n_{\max }(C)+1\right)=0
$$

where the sum is over all the charged chiral superfields, including the PVs, the physical matter superfields $Q^{I}$ and the ghosts $c, c^{\prime}$ and $b$ of the gauge fixing.

Now consider the finite terms in formulæ (C.8) and (C.9). For the matter superfields $\tilde{A}^{\left(n_{\max }\right)}=|\varphi|^{2-2 n_{\max }} A^{\left(n_{\max }\right)}$ where $A^{\left(n_{\max }\right)}$ is a constant of the order $O\left(\Lambda^{-2 n_{\max }}\right)$ while $\tilde{A}^{(0)}=|\varphi|^{2} \exp \left(\frac{-\kappa^{2}}{3} K\right) Z$. Assuming all the matter superfields to 
be light compared to the renormalization momentum $p$, we can write the total of their finite contributions to the $\left\{g_{a}^{-2}(p)\right\}$ as

$$
\begin{aligned}
\sum_{r}^{\text {matter }} \frac{T_{a}(r)}{16 \pi^{2}}( & n_{r}\left(\frac{2}{3} \kappa^{2} K-4 n_{\max }(\text { matter })[\log \Lambda+\log |\langle\varphi\rangle|]-\log p^{2}\right) \\
& \left.-2 \log \operatorname{det} Z^{(r)}\right),
\end{aligned}
$$

plus a constant that depends on the dimensionless parameter $\Lambda^{2 n_{\max }} A^{\left(n_{\max }\right)}$ of the WRS.

For the Faddeev-Popov ghosts $n_{\max }=0$ and $\tilde{A}^{(0)}=|\varphi|^{2}$; for the NielsenKallosh ghost $n_{\max }=n_{\max }\left(\right.$ gauge), $\tilde{A}^{\left(n_{\max }\right)}=|\varphi|^{-4-2 n_{\max }} B^{\left(n_{\max }\right)}$ where $B^{\left(n_{\max }\right)}$ is a constant of the order $O\left(\Lambda^{-2 n_{\max }}\right)$ and $\tilde{A}^{(0)}=|\varphi|^{-4} R e f_{a}$. All ghosts are massless; thus, the total of their finite contributions to the $\left\{g_{a}^{-2}(p)\right\}$ is

$$
\frac{T\left(G_{a}\right)}{16 \pi^{2}}\left(4 n_{\max }(\text { gauge })[\log \Lambda+\log |\langle\varphi\rangle|]+3 \log p^{2}+2 \log \operatorname{Re} f_{a}\right)+\text { const. }
$$

For the Pauli-Villars superfields, $\tilde{A}^{\left(n_{\max }\right)} /|\widetilde{M}|=|\varphi|^{-1-2 n_{\max }} \times$ a constant of the order $O\left(\Lambda^{-1-2 n_{\max }}\right)$, regardless of the Weyl type of a particular PVs. Hence, the total finite contribution of the PVs to the $\left\{g_{a}^{-2}(p)\right\}$ is

$$
-\sum_{\mathcal{P}} \frac{ \pm T_{a}(\mathcal{P})}{16 \pi^{2}}\left(4 n_{\max }(\mathcal{P})+2\right)[\log \Lambda+\log |\varphi|]+\text { const. }
$$

Using eq. (B.7), we can re-express this sum over the Pauli-Villars supermultiplets as a sum over the matter and ghost supermultiplets. Combining the result with eqs. (C.11) and (C.12), we obtain

$$
\begin{aligned}
\left\{g_{a}^{-2}(p)\right\}^{1-\text { loop }} & =\operatorname{Re} f_{a}+\text { const } \\
& +\sum_{r}^{\text {matter }} \frac{T_{a}(r)}{8 \pi^{2}}\left(n_{r}\left(\frac{2}{3} \kappa^{2} K+\log \frac{\Lambda^{2}}{p^{2}}+2 \log |\varphi|\right)-2 \log \operatorname{det} Z^{(r)}\right) \\
& +\frac{T\left(G_{a}\right)}{16 \pi^{2}}\left(-3 \log \frac{\Lambda^{2}}{p^{2}}-6 \log |\varphi|+2 \log \operatorname{Re} f_{a}\right) .
\end{aligned}
$$


Note that the ultraviolet types $n_{\max }$ of any of the charged superfields cancel out of this formula; indeed, only the constant (i.e., momentum-, moduli- and $\varphi$-independent) term in eq. (C.14) is affected in any way by any of the intricacies of the Warr's regularization scheme. The actual value of this constant term depends on the scheme's dimensionless parameters $\Lambda^{2 n_{\max }} A^{\left(n_{\max }\right)}, \Lambda^{2 n_{\max }} B^{\left(n_{\max }\right)}$, $\Lambda^{2 n_{\max }} A_{\mathcal{P}}^{\left(n_{\max }\right)}$ and $M_{\mathcal{P}} / \Lambda$; these parameters are freely adjustable since the regularizing properties of the Warr's cutoff do not depend on their values. By adjusting the cutoff's parameters it is easy to eliminate the overall constant in eq. (C.14); henceforth, we shall assume that the cutoff's parameters are indeed adjusted in this way.

Our next step is to re-organize eq. (C.14) by grouping together all the momentum, $\varphi$ or Kähler dependent terms. Using the $\varphi$-dependent Kähler function $\widetilde{K}$ instead of $K$, we have

$$
\begin{aligned}
\left\{g_{a}^{-2}(p)\right\}^{1-\text { loop }}=\operatorname{Re} f_{a} & +\frac{3 \mathbf{c}_{a}}{8 \pi^{2}} \operatorname{Re} \log \varphi+\frac{\mathbf{c}_{a} \kappa^{2}}{16 \pi^{2}} \widetilde{K}+\frac{\mathbf{b}_{a}}{16 \pi^{2}}\left(\log \frac{\Lambda^{2}}{p^{2}}-\frac{\kappa^{2} \widetilde{K}}{3}\right) \\
& +\frac{T\left(G_{a}\right)}{8 \pi^{2}} \log \operatorname{Re} f_{a}-\sum_{r}^{\operatorname{matter}} \frac{T_{a}(r)}{8 \pi^{2}} \log \operatorname{det} Z^{(r)},
\end{aligned}
$$

where $\mathbf{b}_{a}$ and $\mathbf{c}_{a}$ are as in eqs. (3.3) and (2.21). Formula (C.15) relates the effective gauge couplings of an EQFT to the bare Lagrangian couplings of the theory regularized in a manifestly locally supersymmetric, background-gauge invariant and super-Weyl invariant manner. Before we proceed with further analysis of this formula, however, we would like to turn our attention to the Wilsonian gauge couplings.

The price of the super-Weyl invariance of the UV regulators we have used so far is its $\varphi$ dependence, which obscures the $\varphi$ and moduli dependence of the Wilsonian couplings of the theory. As discussed in section 2.3, we identify the Wilsonian couplings with the bare Lagrangian couplings of a theory whose UV regulator 
is totally independent of the moduli and of the $\varphi$ while retaining as many of the symmetries of the theory as possible under circumstances. For the problem at hand, this means that the regulator should be locally supersymmetric and backgroundgauge invariant but the super-Weyl invariance has to be given up. Consequently, the bare couplings of the theory may acquire non-classical dependences on the Weyl compensators $\varphi$ and $\bar{\varphi}$; in particular, we may have $\varphi$-dependent bare gauge couplings $f_{a}$.

It is very easy to render the Warr's regularization scheme $\varphi$-independent at the expense of the super-Weyl invariance: One simply erases all appearances of $\varphi$ and $\bar{\varphi}$ in eqs.(B.9) through (B.18). The implications of this erasure for the way we calculate the effective gauge couplings are also simple: Everything works exactly as before, except for the use of the $\varphi$-independent $A^{(n)}$, and $M$ instead of the $\tilde{A}^{(n)}$ and the $\widetilde{M}$. Only the lowest-derivative coefficients $\exp \left(-\frac{1}{3} \kappa^{2} \widetilde{K}\right) Z$ for the matter superfields $Q^{I}$ remain $\varphi$-dependent since they are part of the classical Lagrangian of the theory rather than its UV regulator. Therefore, repeating the steps that lead to eq. (C.15) almost verbatim, we arrive at

$$
\begin{aligned}
\left\{g_{a}^{-2}(p)\right\}^{1-\text { loop }}=\operatorname{Re} f_{a} & +\frac{\mathbf{c}_{a} \kappa^{2}}{16 \pi^{2}} \widetilde{K}+\frac{\mathbf{b}_{a}}{16 \pi^{2}}\left(\log \frac{\Lambda^{2}}{p^{2}}-\frac{\kappa^{2} \widetilde{K}}{3}\right) \\
& +\frac{T\left(G_{a}\right)}{8 \pi^{2}} \log \operatorname{Re} f_{a}-\sum_{r}^{\text {matter }} \frac{T_{a}(r)}{8 \pi^{2}} \log \operatorname{det} Z^{(r)} .
\end{aligned}
$$

The only difference between eqs. (C.15) and (C.16) is that the latter lacks the $\left(3 \mathbf{c}_{a} / 8 \pi^{2}\right)$ Re $\log \varphi$ term, plus the fact that the bare couplings involved in the two formulæ differ from each other; this difference is the finite renormalization effect of changing the UV regulators of the theory. Identifying the bare gauge coupling in eq. (C.16) with the true Wilsonian gauge coupling $\tilde{f}_{a}^{W}(\Phi, \varphi)$ and re-naming the $\varphi$-independent bare gauge coupling in eq. (C.15) ' $f_{a}^{W}(\Phi)$ ', we have

$$
\tilde{f}_{a}^{W}(\Phi, \varphi)=f_{a}^{W}(\Phi)+\frac{3 \mathbf{c}_{a}}{8 \pi^{2}} \log \varphi
$$


As described in section 2.3, neither $f_{a}^{W}$ nor $\tilde{f}_{a}^{W}$ renormalize beyond the one-loop level ( $c f$. Appendix A); therefore, eq. (C.17) holds without any modifications to all orders of the perturbation theory.

Finally, let us consider the momentum dependence of the effective gauge couplings. In eqs. (C.15) and (C.16), the reference momentum appears in combination

$$
\log \frac{\Lambda^{2}}{p^{2} \equiv \eta^{m n} p_{m} p_{n}}-\frac{\kappa^{2} \widetilde{K}}{3}
$$

where $\eta^{m n}=\operatorname{diag}(-,+,+,+)$ is the flat un-rescaled Minkowski metric; this unrescaled metric is implicit in the rigid-SUSY formalism we used throughout this section. On the other hand, supergravitationally trivial background of a locally supersymmetric EQFT means conventionally normalized flat vielbein $E_{M}^{A}$, which for generic moduli $\operatorname{VEVs}\left\langle\Phi^{i}\right\rangle$ and generic $\langle\varphi\rangle$ implies that the physical spacetime metric is

$$
g_{m n}=\eta_{m n} \exp \left(-\frac{1}{3} \kappa^{2} \widetilde{K}\right)
$$

( $c f$. eq. (2.8)) rather than the un-rescaled $\eta_{m n}$. Therefore, the Kähler term in eq. (C.18) is the artifact of using the wrong metric: In terms of the physical metric $g_{m n}$,

$$
\log \frac{\Lambda^{2}}{\eta^{m n} p_{m} p_{n}}-\frac{\kappa^{2} \widetilde{K}}{3}=\log \frac{\Lambda^{2}}{p_{\text {phys }}^{2} \equiv g^{m n} p_{m} p_{n}} .
$$

(In the Wess-Zumino gauge (2.18) $g^{m n}=\eta^{m n}$ and eq. (C.20) is a tautology, but in other super-Weyl gauges one has to distinguish between the physical momentum square and the $\eta^{m n} p_{m} p_{n}$.) Thus, the physical meaning of eqs. (C.15) and (C.16) is

$$
\begin{aligned}
& \operatorname{Re} \tilde{f}_{a}^{W}+\frac{\mathbf{c}_{a} \kappa^{2}}{16 \pi^{2}} \widetilde{K}+\frac{\mathbf{b}_{a}}{8 \pi^{2}} \log \Lambda=\operatorname{Re} f_{a}^{W}+\frac{\mathbf{c}_{a} \kappa^{2}}{16 \pi^{2}} K+\frac{\mathbf{b}_{a}}{8 \pi^{2}} \log \Lambda=F_{a} \\
& =\left\{g_{a}^{-2}(p)\right\}^{1-\text { loop }}+\frac{\mathbf{b}_{a}}{16 \pi^{2}} \log p_{\text {phys }}^{2}-\frac{T\left(G_{a}\right)}{8 \pi^{2}} \log g_{a}^{-2}+\sum_{r}^{\text {matter }} \frac{T_{a}(r)}{8 \pi^{2}} \log \operatorname{det} Z^{(r)}
\end{aligned}
$$

which is precisely the one-loop approximation to the eqs. (3.4) and (3.7). 


\section{APPENDIX D \\ Effective Axionic Couplings}

Classically, moduli-dependence of the $\theta_{a}(\Phi, \bar{\Phi})$ - the coefficients of the CP-odd terms $\operatorname{tr}_{a}(F \tilde{F})$ in the gauge-field Lagrangian - gives rise to the axionic couplings of the moduli scalars. In a quantum theory, derivative couplings of the moduli to the charged fermions also lead to the same effect: The 3-point Green's function for two gauge bosons, $A_{m}^{(a)}\left(p_{1}\right)$ and $A_{n}^{(a)}\left(p_{1}\right)$, and a modulus scalar $\Phi^{i}$ (or $\bar{\Phi}^{\imath}$ ), acquires a CP-odd component $\left(1 / 16 \pi^{2}\right) \epsilon^{m n k l} p_{1 k} p_{2 l}\left\{\theta_{a, i(\text { or } \bar{\imath})}\right\}$. In supersymmetric theories, the effective axionic couplings $\left\{\theta_{a, i(\text { or } \bar{\imath})}(\langle\Phi\rangle,\langle\bar{\Phi}\rangle\}\right.$ are related to the moduli-dependence of the effective gauge couplings ${ }^{[8,35]}$ :

$$
\begin{aligned}
& \left\{\theta_{a, i}(\langle\Phi\rangle,\langle\bar{\Phi}\rangle)\right\}=+8 \pi^{2} i \frac{\partial\left\{g_{a}(\langle\Phi\rangle,\langle\bar{\Phi}\rangle)\right\}^{-2}}{\partial\left\langle\Phi^{i}\right\rangle}, \\
& \left\{\theta_{a, \bar{\imath}}(\langle\Phi\rangle,\langle\bar{\Phi}\rangle)\right\}=-8 \pi^{2} i \frac{\partial\left\{g_{a}(\langle\Phi\rangle,\langle\bar{\Phi}\rangle)\right\}^{-2}}{\partial\left\langle\bar{\Phi}^{\bar{\imath}}\right\rangle} .
\end{aligned}
$$

The basis for these relations is SUSY on the level of Green's functions, and as long as it is unbroken, it does not matter exactly how the EQFT is quantized or whether SUSY is rigid or local. ${ }^{[8]}$

Combining eqs. (D.1) and (3.4), we obtain

$$
\begin{aligned}
& \left(1-\frac{T\left(G_{a}\right)}{8 \pi^{2}}\left\{g_{a}\right\}^{2}\right) \cdot\left\{\theta_{a, i}\right\}=+i \frac{\partial}{\partial\left\langle\Phi^{i}\right\rangle}\left[8 \pi^{2} F_{a}-\sum_{r} T_{a}(r) \log \operatorname{det}\left\{Z^{(r)}\right\}\right] \\
& \left(1-\frac{T\left(G_{a}\right)}{8 \pi^{2}}\left\{g_{a}\right\}^{2}\right) \cdot\left\{\theta_{a, \bar{\imath}}\right\}=-i \frac{\partial}{\partial\left\langle\bar{\Phi}^{\bar{\imath}}\right\rangle}\left[8 \pi^{2} F_{a}-\sum_{r} T_{a}(r) \log \operatorname{det}\left\{Z^{(r)}\right\}\right]
\end{aligned}
$$

again, it does not matter whether SUSY is rigid or local or whether it is manifest in the Wilsonian action and the cutoff. Therefore, in order to completely determine the moduli dependence of the renormalization group integrals $F_{a}$, all we have to do is to calculate the effective axionic couplings, and we can perform this calculation in any formalism we like. (Similar computations are also performed in refs. [9-11].) 
At the one-loop level of analysis (which is sufficient to completely determine $F_{a}$ ), the easiest way to compute an effective axionic coupling is to use component fields and ordinary Feynman rules. There are three contributions to $\left\{\theta_{a, i}\right\}$ : the tree-level term $\partial \theta_{a}^{W} / \partial \Phi^{i}$; the renormalization of that term due to loops of gauge bosons; and, finally, the axial anomaly graphs involving charged fermions $\Psi_{\alpha}^{I}$ and $\lambda_{\alpha}^{(a)}$. Assuming all such fermions retained in an EQFT are massless, we have

$$
\left\{\theta_{a, i}\right\}^{1-\text { loop }}=\frac{\partial \theta_{a}^{W}}{\partial \Phi^{i}} \cdot\left(1+\frac{T\left(G_{a}\right)}{4 \pi^{2}} g^{2}\right)-2 i \sum_{r}^{\substack{\text { all charged } \\ \text { fermions }}} T_{a}(r) \operatorname{Tr} \Gamma_{i}^{(r)},
$$

where $\Gamma_{i}^{(r)}$ is the matrix of couplings of $\partial_{m} \Phi^{i}$ to the 'flavor' currents of charged fermions; a similar formula holds for the axionic couplings of the anti-chiral moduli $\overline{\Phi^{\imath}}$. To make our notations clear, let us write down the terms in the Wilsonian Lagrangian of the EQFT that involve two charged fermionic fields, one left-handed and one right-handed:

$$
L_{\bar{f} f}=\frac{i}{2} Z_{\bar{I} J}\left(\bar{\Psi}^{\bar{I}} \overleftrightarrow{\not} \Psi^{J}\right)+\frac{i}{2}(\operatorname{Re} f)_{(a)(b)}^{-1}\left(\bar{\lambda}^{(a)} \overleftrightarrow{\not} \lambda^{(b)}\right)
$$

where $\not \equiv \gamma^{m} \nabla_{m}, \nabla_{m}$ being the covariant derivative containing gravitational, gauge and flavor connections:

$$
\nabla_{m}=\partial_{m}+\frac{1}{2} \omega_{m}^{a b} \sigma_{a b}-i A_{m}^{(a)} T_{(a)}+\left(\partial_{m} \Phi^{i}\right) \Gamma_{i}+\left(\partial_{m} \bar{\Phi}^{\imath}\right) \Gamma_{\bar{\imath}}
$$

In this formula, $\Gamma_{i}$ and $\Gamma_{\bar{\imath}}$ are matrices carrying 'flavor' indices, i.e., $\left(\Gamma_{i} \Psi\right)^{J}=$ $\Gamma_{i K}^{J} \Psi^{K}$, etc.; $\Gamma_{i}^{(r)}$ in eq. (D.3) is the restriction of the full $\Gamma_{i}$ matrix to fermions transforming like $r$ under the gauge group.

Formula (D.3) presumes background gauge invariance of the quantum theory but it does not need manifest SUSY. Therefore, we can obtain the $\Gamma_{i}$ and $\Gamma_{\bar{\imath}}$ matrices of a locally supersymmetric EQFT by simply looking up the component-field 
Lagrangian in any standard reference on SUGRA and identifying the appropriate terms as belonging to (D.4). Using as our source ref. [5], we have, in matrix notations,

$$
\begin{aligned}
\Gamma_{i}^{(\Psi)} & =\frac{1}{2 Z} \frac{\partial Z}{\partial \Phi^{i}}-\frac{\kappa^{2}}{4} \frac{\partial K}{\partial \Phi^{i}}, \\
\Gamma_{\bar{\imath}}^{(\Psi)} & =\frac{-1}{2 Z} \frac{\partial Z}{\partial \bar{\Phi}^{\bar{\imath}}}+\frac{\kappa^{2}}{4} \frac{\partial K}{\partial \bar{\Phi}^{\bar{\imath}}} \\
\Gamma_{i}^{(\lambda)} & =\frac{1}{2\left(f+f^{*}\right)} \frac{\partial f}{\partial \Phi^{i}}+\frac{\kappa^{2}}{4} \frac{\partial K}{\partial \Phi^{i}}, \\
\Gamma_{\bar{\imath}}^{(\lambda)} & =\frac{-1}{2\left(f+f^{*}\right)} \frac{\partial f^{*}}{\partial \bar{\Phi}^{\bar{\imath}}}-\frac{\kappa^{2}}{4} \frac{\partial K}{\partial \bar{\Phi}^{\bar{\imath}}}
\end{aligned}
$$

Rigidly supersymmetric EQFTs have similar $\Gamma_{i}$ matrices, but without the Kähler terms.

At this point, all that remains to do is some straightforward algebra. Substituting $\theta_{a}^{W}=-8 \pi^{2} \operatorname{Im} f_{a}^{W}, g_{a}^{2}=1 / \operatorname{Re} f_{a}^{W}$ and the matrices (D.6) into eq. (D.3) leads to

$$
\begin{aligned}
&\left\{\theta_{a, i}\right\}^{1-\text { loop }}=i\left(4 \pi^{2}+T\left(G_{a}\right) g_{a}^{2}\right) \frac{\partial f_{a}^{W}}{\partial \Phi^{i}}-\frac{i}{2} T\left(G_{a}\right)\left(g_{a}^{2} \frac{\partial f_{a}^{W}}{\partial \Phi^{i}}+\kappa^{2} \frac{\partial K}{\partial \Phi^{i}}\right) \\
&+\frac{i}{2} \frac{\partial}{\partial \Phi^{i}} \sum_{r} T_{a}(r)\left[n_{r} \kappa^{2} K-2 \log \operatorname{det} Z^{(r)}\right] \\
&=i\left(8 \pi^{2}+T\left(G_{a}\right) g_{a}^{2}\right) \frac{\partial \operatorname{Re} f_{a}^{W}}{\partial \Phi^{i}} \\
&+i \frac{\partial}{\partial \Phi^{i}}\left[\mathbf{c}_{a} \cdot \frac{1}{2} \kappa^{2} K-\sum_{r} T_{a}(r) \log \operatorname{det} Z^{(r)}\right]
\end{aligned}
$$

and ditto for the $\left\{\theta_{a, \bar{l}}\right\}$. Note how the coefficients of all the Kähler terms assemble into precisely the $\mathbf{c}_{a}$ defined in eq. (2.21). Now we substitute eqs. (D.7) and their

* Actually, not all standard references can be used verbatim but only those following the "American" convention for the Weyl rescaling in which fermionic fields are rescaled but their phases remain unchanged; in the super-Weyl invariant language, this convention corresponds to $\arg \varphi=0$ and hence $\theta_{a}^{W}=-8 \pi^{2} \operatorname{Im} \tilde{f}_{a}^{W}=-8 \pi^{2} \operatorname{Im} f_{a}^{W}$. In the "European" phase convention, the relation between the properly defined Wilsonian $\theta$ angles and the imaginary parts of $f_{a}$ is not so straightforward. 
complex conjugates into eqs. (D.2), which immediately gives us

$$
\begin{aligned}
F_{a}(\Phi, \bar{\Phi})-\operatorname{Re} f_{a}^{W}(\Phi)-\mathbf{c}_{a} \frac{\kappa^{2} K(\Phi, \bar{\Phi})}{16 \pi^{2}} \\
=\text { a moduli-independent constant }+O\left(g^{2}\right)
\end{aligned}
$$

The nature of the constant here being obvious from the Wilsonian renormalization of $f_{a}^{W}$, eq. (D.8) is essentially the same as eq. (3.7), except for the $O\left(g^{2}\right)$ terms, which are artifacts of the one-loop approximation to the axionic couplings and should cancel out of more accurate higher-order calculations.

We conclude that eq. (3.7) is quite robust: Any formulation of a locally supersymmetric EQFT in which $\theta_{a}^{W}(\Phi, \bar{\Phi})=-8 \pi^{2} \operatorname{Im} f_{a}^{W}(\Phi)$ for holomorphic $f_{a}^{W}$ would lead to the same relation between $\operatorname{Re} f_{a}^{W}$ and the renormalization group integrals $F_{a}$ for the running effective gauge couplings $\left\{g_{a}(p)\right\}$. In particular, the Kähler term eq. (3.7) is an inalienable feature of the local SUSY; the compensator formalism may be the easiest way to see why this term should be present, but all other ways should lead to the same destination.

\section{REFERENCES}

1. M.A. Shifman and A.I. Vainshtein, Nucl. Phys. B277 (1986) 456.

2. K. G. Wilson and J. G. Kogut, Physics Report 12 (1974), 75; see also J. Polchinski, Nucl. Phys. B231 (1984), 269.

3. M.A. Shifman and A.I. Vainshtein, Nucl. Phys. B359 (1991) 571.

4. E. Cremmer, S. Ferrara, L. Girardello and A. Van Proeyen, Nucl. Phys. B212 (1983) 413.

5. J. Wess and J. Bagger, Supersymmetry and Supergravity (Princeton Unversity Press, 1983). 
6. S. J. Gates, M. Grisaru, M. Roček and W. Siegel, Superspace, Benjamin/Cummings, 1983.

7. H. P. Nilles, Phys. Lett. 180B (1986) 240.

8. L. Dixon, V. Kaplunovsky and J. Louis, Nucl. Phys. B355 (1991) 649.

9. A preliminary version of this article was presented by J. Louis, in the Proceedings of the 2nd International Symposium on Particles, Strings and Cosmology, Boston, MA, March 25-30, 1991, ed. P. Nath und S. Reucroft.

10. J. P. Derendinger, S. Ferrara, C. Kounnas and F. Zwirner, Nucl. Phys. B372 (1992) 145 and Phys. Lett. B271 (1991) 307.

11. G. L. Cardoso and B. Ovrut, Nucl. Phys. B369 (1992) 351; Nucl. Phys. B392 (1993) 315 and CERN preprint CERN-TH.6961/93.

12. T. E. Clark, O. Piguet and K. Sibold, Nucl. Phys. B159 (79) 1;

K. Konishi, Phys. Lett. 135B (1984) 439.

13. J.P. Derendinger, L.E. Ibáñez and H.P. Nilles, Phys. Lett. 155B (1985) 65; M. Dine, R. Rohm, N. Seiberg and E. Witten, Phys. Lett. 156B (1985) 55.

14. for a review see H. P. Nilles, Int. J. Mod. Phys. A5 (1990), 4199, J. Louis, in Proceedings of the 1991 DPF meeting, World Scientific, 1992, and references therein.

15. S. Ferrara, L. Girardello and H. P. Nilles, Phys. Lett. 125B (1983) 457.

16. G. Veneziano and S. Yankielowicz, Phys. Lett. 113B (1982) 231; T.R. Taylor, G. Veneziano and S. Yankielowicz, Nucl. Phys. B218 (1983) 493 ;

T.R. Taylor, Phys. Lett. 164B (1985) 43.

17. I. Affleck, M. Dine and N. Seiberg, Phys. Rev. Lett. 51 (1984) 1026, Nucl. Phys. B241 (1984) 493 and Nucl. Phys. B256 (1985) 557. 
18. V. Novikov, M.A. Shifman, A.I. Vainshtein and V.I. Zakharov, Nucl. Phys. B260 (1985) 157;

M.A. Shifman and A.I. Vainshtein, Nucl. Phys. B296 (1988) 445.

19. D. Amati, K. Konishi, Y. Meurice, G. Rossi and G. Veneziano, Phys. Rep. $162(1988) 169$.

20. A. Font, L.E. Ibáñez, D. Lüst and F. Quevedo, Phys. Lett. B245 (1990) 401.

21. S. Ferrara, N. Magnoli, T. Taylor and G. Veneziano, Phys. Lett. B245 (1990) 409.

22. H. P. Nilles and M. Olechowski, Phys. Lett. B248 (1990) 268.

23. P. Binétruy and M. K. Gaillard, Phys. Lett. B253 (1991) 119 and Nucl. Phys. B358 (1991) 121.

24. D. Lüst and T. Taylor, Phys. Lett. B253 (1991) 335;

B. de Carlos, J. A. Casas and C. Muñoz, Phys. Lett. B263 (1991) 248 and Nucl. Phys. B399 (1993) 621.

25. A. Masiero, R. Pettorino, M. Roncadelli and G. Veneziano, Nucl. Phys. B261 (1985) 633.

26. V. Kaplunovsky and J. Louis, Phys. Lett. B306 (1993) 269.

27. P. Binetruy, G. Girardi and R. Grimm, Phys. Lett. B265 (1991) 111;

P. Adamietz, P. Binetruy, G. Girardi and R. Grimm, Nucl. Phys. B401 (1993) 257.

28. V. Kaplunovsky and J. Louis, in preparation.

29. T. Kugo and S. Uehara, Nucl. Phys. B222 (1983) 125.

30. P. Howe and R. Tucker, Phys. Lett. 80B, 138 (1978).

31. P. Binetruy, G. Girardi and R. Grimm, LAPP preprint LAPP-TH-275-90 and references therein. 
32. I. Jack and D. R. T. Jones, Phys. Lett. B258 (1991) 382; P. West, Phys. Lett. B258 (1991) 375.

33. S. Cecotti, S. Ferrara, L. Girardello and M. Porrati Phys. Lett. 164B (1985) 64.

34. V. Novikov, M.A. Shifman, A.I. Vainshtein and V.I. Zakharov, Nucl. Phys. B229 (1983) 381.

35. V. Kaplunovsky, in preparation.

36. I. Antoniadis, K. Narain and T. Taylor, Phys. Lett. B267 (1991) 37;

I. Antoniadis, E. Gava and K. Narain, Nucl. Phys. B383 (1992) 93.

37. S. Ferrara, C. Kounnas, D. Lüst and F. Zwirner, Nucl. Phys. B365 (1991) 431.

38. P. Mayr and S. Stieberger, Nucl. Phys. B407 (1993) 725 and Max Planck preprint MPI-Ph/93-14.

39. M. K. Gaillard and T. Taylor, Nucl. Phys. B381 (1992) 577.

40. M. Bershadsky, S. Cecotti, H. Ooguri and C. Vafa, Nucl. Phys. B405 (1993) 279 and Harvard preprint HUTP-93-A025.

41. S. Weinberg, Phys. Lett. B91 (1980) 51.

42. V. Kaplunovsky, Nucl. Phys. B307 (1988) 145.

43. see J. Lopez and D.V. Nanopoulos, Phys. Rev. D47 (1993) 2468 and references therein.

44. B. Greene, K. Kirklin, P. Miron and G.G. Ross, Nucl. Phys. B278 (1986) 667 and Nucl. Phys. B292 (1987) 292.

45. E. Witten, Nucl. Phys. B188 (1981) 513.

46. M. Cvetič, A. Font, L.E. Ibáñez, D. Lüst and F. Quevedo, Nucl. Phys. B361 (1991) 194. 
47. P. Candelas, G. Horowitz, A. Strominger and E. Witten, Nucl. Phys. B258 (1985) 46.

48. N. Seiberg, Rutgers preprint RU-93-45.

49. L. Ibáñez, W. Lerche, D. Lüst and S. Theisen, Nucl. Phys. B352 (1991) 435.

50. L. Dixon, J. Harvey, C. Vafa and E. Witten, Nucl. Phys. B261 (1985) 678; Nucl. Phys. B274 (1986) 185.

51. M. Dine and N. Seiberg, Phys. Lett. 162B (1985) 299.

52. N.V. Krasnikov, Phys. Lett. 193B (1987) 37;

L. Dixon, in the Proccedings of the 15th A.P.S. D.P.F. Meeting, Houston, 1990;

J. A. Casas, Z. Lalak, C. Muñoz and G.G. Ross, Nucl. Phys. B347 (1990) 243 ;

T. Taylor, Phys. Lett. B252 (1990) 59.

53. A. de la Macorra and G. Ross, Nucl. Phys. B404 (1993) 321.

54. P. Candelas, P.S. Green and T. Hübsch, Nucl. Phys. B330 (1990) 49.

55. G.D. Coughlan, W. Fischler, E. Kolb, S. Raby and G.G. Ross, Phys. Lett. 131B (1983) 59.

56. T. Banks, D. Kaplan and A. Nelson, Rutgers preprint RU-37 (1993).

57. B. de Carlos, J.A. Casas, F. Quevedo and E. Roulet, CERN preprint CERNTH.6958/93.

58. E.J. Chun, J.E. Kim and H.P. Nilles, Nucl. Phys. B370 (1992) 105.

59. M. Dine and A. Nelson, Phys. Rev. D48 (1993) 1277.

60. B. Warr, Annals of Phys. 183 (1988), p. 1 and p. 59.

61. O. Piguet and K. Sibold, Renormalized Supersymmetry, Birkhäuser, Boston, 1986. 